\title{
VEP estimation of visual acuity: a systematic review
}

\author{
Ruth Hamilton (D) Michael Bach • Sven P. Heinrich • Michael B. Hoffmann • \\ J. Vernon Odom • Daphne L. McCulloch • Dorothy A. Thompson
}

Received: 12 November 2019/Accepted: 5 May 2020/Published online: 2 June 2020

(C) The Author(s) 2020

\begin{abstract}
Purpose Visual evoked potentials (VEPs) can be used to measure visual resolution via a spatial frequency (SF) limit as an objective estimate of visual acuity. The aim of this systematic review is to collate descriptions of the VEP SF limit in humans, healthy and disordered, and to assess how accurately and precisely VEP SF limits reflect visual acuity.

Methods The protocol methodology followed the PRISMA statement. Multiple databases were searched using "VEP" and "acuity" and associated terms, plus hand search: titles, abstracts or full text were reviewed for eligibility. Data extracted included VEP SF limits, stimulus protocols, VEP recording and analysis techniques and correspondence with behavioural acuity for
\end{abstract}

R. Hamilton $(\square)$

Department of Clinical Physics and Bioengineering,

Royal Hospital for Children, NHS Greater Glasgow and

Clyde, Glasgow, UK

e-mail: ruth.hamilton@glasgow.ac.uk

R. Hamilton

College of Medical, Veterinary and Life Sciences,

University of Glasgow, Glasgow, UK

M. Bach · S. P. Heinrich

Eye Center, Medical Center - University of Freiburg,

Faculty of Medicine, University of Freiburg, Freiburg,

Germany

M. B. Hoffmann

Department of Ophthalmology, Otto-von-Guericke

University, Magdeburg, Germany normally sighted healthy adults, typically developing infants and children, healthy adults with artificially degraded vision and patients with ophthalmic or neurological conditions.

Results A total of 155 studies are included. Commonly used stimulus, recording and analysis techniques are summarised. Average healthy adult VEP SF limits vary from 15 to $40 \mathrm{cpd}$, depend on stimulus, recording and analysis techniques and are often, but not always, poorer than behavioural acuity measured either psychophysically with an identical stimulus or with a clinical acuity test. The difference between VEP SF limit and behavioural acuity is variable and strongly dependent on the VEP stimulus and choice of acuity test. VEP SF limits mature rapidly, from 1.5 to $9 \mathrm{cpd}$ by the end of the first month of life to $12-20 \mathrm{cpd}$

M. B. Hoffmann

Center for Behavioral Brain Sciences, Magdeburg, Germany

J. V. Odom

Departments of Ophthalmology and Neuroscience, School of Medicine, West Virginia University, Morgantown, WV, USA

D. L. McCulloch

School of Optometry and Vision Science, University of Waterloo, Waterloo, ON, Canada

D. A. Thompson

The Department of Clinical and Academic

Ophthalmology, Great Ormond Street Hospital for Children, London, UK 
by 8-12 months, with slower improvement to $20-40$ cpd by $3-5$ years. VEP SF limits are much better than behavioural thresholds in the youngest, typically developing infants. This difference lessens with age and reaches equivalence between 1 and 2 years; from around 3-5 years, behavioural acuity is better than the VEP SF limit, as for adults. Healthy, artificially blurred adults had slightly better behavioural acuity than VEP SF limits across a wide range of acuities, while adults with heterogeneous ophthalmic or neurological pathologies causing reduced acuity showed a much wider and less consistent relationship. For refractive error, ocular media opacity or pathology primarily affecting the retina, VEP SF limits and behavioural acuity had a fairly consistent relationship across a wide range of acuity. This relationship was much less consistent or close for primarily macular, optic nerve or neurological conditions such as amblyopia. VEP SF limits were almost always normal in patients with non-organic visual acuity loss.

Conclusions The VEP SF limit has great utility as an objective acuity estimator, especially in pre-verbal children or patients of any age with motor or learning impairments which prevent reliable measurement of behavioural acuity. Its diagnostic power depends heavily on adequate, age-stratified, reference data, age-stratified empirical calibration with behavioural acuity, and interpretation in the light of other electrophysiological and clinical findings. Future developments could encompass faster, more objective and robust techniques such as real-time, adaptive control. Registration International prospective register of systematic reviews PROSPERO (https://www.crd. york.ac.uk/PROSPERO/), registration number CRD42018085666.

Keywords Systematic review - VEP .

Visual acuity - Spatial frequency limit · ISCEV .

Threshold · Sweep VEP

Abbreviations
VEP $\quad$ Visual evoked potential
ssVEP $\quad$ Steady-state VEP

D. A. Thompson

University College London Great Ormond Street Institute of Child Health, London, UK

$\begin{array}{ll}\text { SF } & \text { Spatial frequency } \\ \mathrm{SF}_{\mathrm{f}} & \text { Fundamental SF } \\ \text { deg } & \text { Degree } \\ \text { MAR } & \text { Minimum angle of resolution } \\ \text { logMAR } & \begin{array}{l}\text { Logarithm (base 10) of MAR } \\ \text { cpd }\end{array} \\ & \begin{array}{l}\text { Cycles per degree, also written as cy/deg } \\ \text { or cy/ }\end{array} \\ \text { DFT } & \text { Discrete Fourier transform } \\ \text { SNR } & \text { Signal-to-noise ratio } \\ \text { rps } & \text { Reversals per second } \\ \text { EEG } & \text { Electroencephalogram } \\ \text { LoA } & \text { Limits of agreement } \\ \text { ETDRS } & \text { Early treatment diabetic retinopathy study } \\ & \text { (acuity test) } \\ \text { SEM } & \text { Standard error of the mean } \\ \text { SD } & \text { Standard deviation } \\ \text { ASD } & \text { Autistic spectrum disorder } \\ \text { CP } & \text { Cerebral palsy } \\ \text { PVL } & \text { Periventricular leukomalacia } \\ \text { TAC } & \text { Teller acuity cards } \\ \text { NOVL } & \text { Non-organic vision loss }\end{array}$

\section{Introduction}

Visual acuity, the threshold for resolving high contrast detail by the visual system, is an important clinical assessment, typically measured using subjective tests such as naming letters or symbols on calibrated charts or estimated using behavioural tests based on looking, pointing or matching. These tests require the patient to have adequate cognitive and motor function and to comply with the test process.

Visual evoked potentials (VEPs) are used in patients who cannot or will not reliably complete subjective or behavioural tests and in those with difficulties in perception and recognition to aid in localising defects. VEPs can be used to measure a threshold as a proxy for, or estimate of, visual acuity: such techniques for estimating acuity have been employed for over 40 years [1, 2]. VEP measurement of spatial frequency (SF) limit is objective, requires less cognitive function or cooperation than behavioural tests and does not depend on intact motor responses. However, even if identical targets or stimuli are used, a VEP measurement of SF limit 
and a behavioural acuity test have not assessed the same entity. Differences include:

(1) At the retina, behavioural acuity tests require only a small number of normally functioning cones to resolve a grating [3], while VEPs require contributions from the fovea and perifovea [4].

(2) A supra-threshold stimulus may be perceived but fail to evoke a measurable VEP, at least partly due to the need for sufficient neural populations to act synchronously to generate a VEP detectable by scalp electrodes.

(3) Behavioural tests are self-paced with decisions based on any brief moment of optimal retinal image quality during longer viewing periods containing accommodation and fixation fluctuations [5], while the VEP will be degraded by such fluctuations because of its requirement for sustained recording.

(4) Behavioural acuity tests assess the visual system as well as higher cognitive and often motor functions (target recognised, task understood, relevant motor response such as saccades, pointing or naming), while the VEP assesses cellular activity in the visual cortex and no higher processes.

(5) Behavioural acuity tests use stationary targets, while VEP stimuli are dynamic, with inherent higher visibility [6].

(6) Behavioural acuity is usually defined as the turning point of a psychometric function or similar measure where stimuli can still be perceived, while a VEP SF limit is often defined as zero- or near-zero extrapolated amplitude. Extrapolation may partially address issues (1) and (2).

Such differences mean that VEP SF limits and behavioural measures of acuity are not always in close agreement. However, the agreement is sufficiently consistent and close that, with suitable regard for those patient groups and disorders which are likely to produce exceptions, VEPs are a vital complementary tool for clinical assessment of acuity and may be the only measure available when behavioural testing is not possible or reliable. Systematic differences between behavioural and VEP techniques can be accounted for with appropriate conversion factors.
The aim of this systematic review is to gather and synthesise evidence to address these questions:

(1) What are typical VEP SF limits in humans, in health and in disease, and how are these measured?

(2) How accurately do VEP SF limits reflect visual acuity, i.e. what is the typical difference between VEP SF limits and behavioural acuity measured in the same subjects?

(3) How precise are VEP SF limits, i.e. what is the typical variability?

\section{Methods}

Protocol and registration

This systematic review study protocol was registered with the international prospective register of systematic reviews (PROSPERO), registration number CRD42018085666. Methodology is reported according to the Preferred Reporting Items for Systematic Reviews and Meta-Analyses (PRISMA) statement [7]. Risk of bias is not assessed, as there is no standard outcome measure being compared: the greatly heterogeneous nature of the included studies preclude meaningful comparison of quality. However, factors such as number of subjects included and robustness of techniques employed are qualitatively discussed.

Eligibility criteria, data sources and search strategies

We included articles, conference proceedings or dissertations which describe VEPs used to measure visual acuity in humans of any age, whether patients or healthy individuals. Languages were restricted to those understood by the authors, i.e. English, German and French. Exclusion criteria were: (1) meeting abstracts, review articles or editorials; (2) animal studies; (3) VEPs for communication, e.g. for braincomputer interface (4) higher-level event-related potentials; (5) VEPs used to measure thresholds other than spatial frequency, e.g. contrast sensitivity, stereoacuity, vernier or hyperacuity, colour or motion thresholds.

Two reviewers (RH, VO) independently and systematically searched MEDLINE, EMBASE, 
PsycINFO and ProQuest for studies published between 1975 and May 2019. MeSH terms or equivalent keywords were ("VEP" or "VECP" or "VER" or "visual evoked potential" or "visual evoked cortical potential" or "visual evoked response") and ("acuity" or "visual acuity" or "threshold" or "spatial frequency" or "spatial threshold" or "sweep" or "swept" or "step" or "stepwise"). This search was supplemented by all authors with hand searching, e.g. reference sections of articles, reviews, book chapters, conference proceedings and monographs. Review articles or other pertinent articles pertaining to VEP SF limits were noted separately in order to capture and compare their conclusions.

\section{Study selection and data extraction}

Titles and abstracts were screened to identify potentially eligible studies for inclusion. Where necessary, the full text was reviewed to determine whether a study met the inclusion criteria. Data were extracted from included studies (Fig. 1) using a standardised template. Extracted information included: study design, participant demographics, details of VEP stimulation, acquisition and analysis, details of any concomitant behavioural acuity tests and main findings. Inclusion/exclusion decisions and data extraction for each study were independently reviewed by one author (RH), and any conflicting decisions were resolved through discussion.

Synthesis of results and summary measures

Where it was possible to compare studies, typical adult VEP SF limits were noted, with summarised findings for effects of different stimulus and acquisition parameters, and threshold estimation techniques. Correspondence of typical adult VEP SF limits with behavioural thresholds (either psychophysically to stimuli identical to the VEP stimuli or using clinical acuity tests) was compiled. VEP SF limits for typically developing infants and children were used to map VEP SF limit maturation; where concomitant behavioural acuities were also measured, correspondence between VEP SF limits and behavioural thresholds was compiled.

Studies reporting the effects of poorer-than-normal acuity on the VEP SF limit were documented, including healthy adults with artificially degraded vision and adult and paediatric patients with ophthalmic or neurological pathologies. Specific note was made of evidence supporting the extent of disparity between VEP SF limit and behavioural acuity for particular conditions.

For some studies, data were available only in figures, rather than explicitly stated in tables or text: if possible, such data were extracted using web-based tools [8]. Extracted data were summarised in tabular form. If numerical pooling was not possible, we generated a set of statements to represent the body of literature reviewed.

To aid clarity, terminology conventions were observed for descriptions of acuity and related measures: "good", "better", "poor" or "poorer" were used in preference to "high", higher", "low" or "lower", since some units, e.g. $\log _{10}$ of the minimum angle of resolution (MAR) (logMAR), are such that low numerical values are attributed to high performance acuities. Pattern element size was described as "coarse" or "fine" in preference to "high" or "low" since SF units, e.g. cycles per degree (cpd), and element size units, e.g. minutes of arc (') have an inverse relation, and therefore opposite meanings of "low" and "high".

For clarity, the most commonly used spatial patterns for evoking VEPs are described in Table 1, including formulae for calculating their fundamental $\mathrm{SF}\left(\mathrm{SF}_{\mathrm{f}}\right)$ in cpd, and expressing VEP SF limits on a $\log \mathrm{MAR}$ scale. A checkerboard's $\mathrm{SF}_{\mathrm{f}}$ is one check diagonal [9, 10]: if checkwidth is used instead of diagonal, the checkerboard's $\mathrm{SF}_{\mathrm{f}}$ is underestimated by $0.15 \mathrm{log}$ units. Where this was evident in data extracted from studies employing checkerboards, values were adjusted.

Multiple terms have been used to describe the performance limit as measured by VEPs, e.g. VEP SF threshold, VEP acuity, VEP acuity estimate, etc.: here, we have elected to use the term "VEP SF limit".

\section{Results}

Included studies

The process of literature review including numbers of records searched, screened, included and excluded is shown in Fig. 1. A total of 329 full-text articles were screened and given a hashtag number: 155 of these 
Table 1 Common pattern VEP stimuli: definitions, and formulae for conversion between spatial frequency units

\begin{tabular}{|c|c|c|c|c|c|}
\hline Spatial pattern & Illustration & $\begin{array}{l}\text { Fundamental spatial period } \\
\text { (angular, minutes of } \operatorname{arc}\left({ }^{\prime}\right) \text { ) }\end{array}$ & $\begin{array}{l}\text { Fundamental spatial } \\
\text { frequency }\left(\mathrm{SF}_{\mathrm{f}}, \mathrm{cpd}\right)\end{array}$ & $\begin{array}{l}\text { Log scale, i.e. } \\
\log \text { MAR if a } \\
\text { threshold }\end{array}$ & Notes \\
\hline Sinusoidal grating & & $\begin{array}{l}\text { One cycle } \\
=\text { separation of } 2 \\
\text { neighbouring luminance } \\
\text { peaks or troughs } \\
=X\end{array}$ & $\mathrm{SF}_{\mathrm{f}}=\frac{60}{X}$ & $=\log _{10}\left[\frac{X}{2}\right]$ & $\begin{array}{l}\text { No higher SF } \\
\text { harmonics }\end{array}$ \\
\hline $\begin{array}{l}\text { Square- } \\
\text { wave grating (bars) }\end{array}$ & & $\begin{array}{l}\text { One cycle } \\
=\text { a bar pair } \\
=2 \times \text { barwidth }\left(w_{b}\right)\end{array}$ & $\mathrm{SF}_{\mathrm{f}}=\frac{60}{2 \times w_{b}}$ & $=\log _{10}\left[w_{b}\right]$ & $\begin{array}{l}\text { Multiple higher } \\
\text { SF harmonics }\end{array}$ \\
\hline Checkerboard & $\begin{array}{c}\sqrt{2} \times w_{c} \\
+8\end{array}$ & $\begin{array}{l}\text { One cycle } \\
=\text { a check diagonal } \\
=\sqrt{2} \times \text { checkwidth }\left(w_{c}\right)\end{array}$ & $\mathrm{SF}_{\mathrm{f}}=\frac{60}{\sqrt{2} \times w_{c}}$ & $=\log _{10}\left[\frac{\sqrt{2} \times w_{c}}{2}\right]$ & $\begin{array}{l}\text { Fundamental SF is } \\
\text { obliquely } \\
\text { oriented } \\
\text { Multiple higher } \\
\text { SF harmonics }\end{array}$ \\
\hline
\end{tabular}

Note logarithmic scale units for describing a pattern's SF, even at VEP SF limit, does not necessarily equate to that behavioural acuity as $\log$ MAR

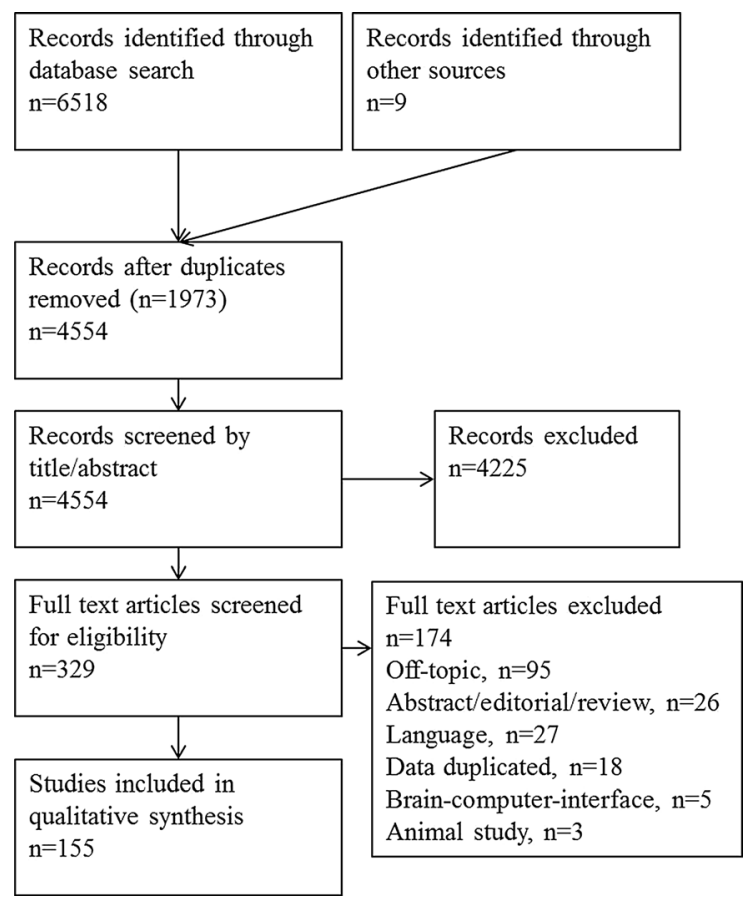

Fig. 1 PRISMA diagram illustrating systematic review process of literature search, screen, inclusion and exclusion

which met the criteria specified in "Methods" section were included in the systematic review. These 155 are indicated in the reference list with their hashtag number at the end of the entry, e.g. (\#24): corresponding reference numbers can be found via a text search function. The hashtags also appear in several figure legends and tables.

\section{Overview of VEP SF limit techniques}

Techniques have converged somewhat over the decades. Stimuli are usually medium-to-high contrast (40-100\%, Michelson), black and white patterns with moderately high $\left(>40 \mathrm{~cd} \mathrm{~m}^{-2}\right)$ mean luminance. Both checkerboards and gratings (horizontal and vertical) are widely used. Field size shows high variability depending on the application, e.g. adult or infant studies. Most studies employ steady-state VEPs (ssVEPs-frequency components with constant amplitude and phase [11]), high stimulation rates and frequency domain analysis for fast, objective signal detection. A minority of studies describe transient VEPs subjectively analysed in the time domain. The underlying brain mechanisms return to a resting state before each re-stimulation for transient VEPs and so around 1 min of constant fixation is needed per stimulus condition for an adequately reproducible recording.

Single channel recordings are most commonly reported, with an active electrode placed over the occipital cortex, and a reference electrode often placed close by. Enhanced success with recovering small 
VEPs has been reported with Laplacian-type montages of three occipital electrodes closely placed. Given that analysis is conducted in the frequency domain, amplifier bandpass is usually kept relatively open. Most commonly, a discrete Fourier transform (DFT) is applied at the stimulation frequency ( $\mathrm{Hz}$ for on/ offsets; rps for reversal stimuli). The significance of the response at the stimulation frequency for each pattern size is objectively decided by adequate signalto-noise ratio (SNR) or by a statistic based on phase, or on magnitude, or on combined magnitude and phase reaching significance.

Most commonly, these data are then used to derive a post hoc VEP SF limit by plotting a magnitude versus SF function, often on linear-linear plots. This function typically shows a descending, approximately linear limb at the finest stimuli: significant data points on this limb are fitted with a regression line which is extrapolated to some baseline, usually zero magnitude or a noise level, to estimate the VEP SF limit. An alternative technique, sometimes employed when linear extrapolation is not feasible, is to declare the VEP SF limit as the finest SF evoking a significant VEP.

While earlier workers sampled VEPs to continuously changing SF, a true "sweep VEP", current implementations are based on discrete SF steps, with each SF presented for around a second. The term "sweep VEP" is nonetheless widely retained, although "stepwise sweep" or "sampled sweep" is also used synonymously. SFs are sampled either linearly or exponentially (linearly spaced on a logarithmic scale). The number of SFs presented varies widely, from as few as three or four to about 20 . Their temporal order is almost always fixed, with the direction of change sometimes from fine-to-coarse, but more often from coarse-to-fine patterns. Occasionally a quasi- or pseudo-randomised order is used but real-time, adaptive techniques are seldom implemented.

VEP SF limits in normally sighted adults

To establish typical VEP SF limits, included papers were reviewed for those which reported VEP SF limits in normal or healthy adults wearing any required refractive correction. Papers were included where VEP SF limits were stated or could be extracted. Average limits vary from 15 to $40 \mathrm{cpd}$. Methods are too diverse to relate different stimulation or analysis techniques to differences in limits (Fig. 2). Where extrapolation techniques are used, VEP SF limits can be beyond the finest SF viewed [12-17]. Many of the studies may include a ceiling effect since the VEP SF limit was beyond the range of tested SFs. Where SFs viewed or available extend to finer values than the VEP SF limit, i.e. bracket the electrophysiological limit and presumably eliminate the possibility of a ceiling effect, average VEP SF limits are slightly higher [18-33]. Several studies give examples of individuals with VEP SF limits of $\geq 40 \mathrm{cpd}$ [12, 18, 19, 34-37] suggesting this as a suitable upper limit for subjects where a normal VEP SF limit is possible.

We identified only one study which described VEP SF limits in older adults [38]. Both behavioural acuity and VEP SF limits peaked around 20 years of age (Landolt C $-0.20 \log$ MAR, VEP SF limit $44 \mathrm{cpd}$ $(-0.17 \log \mathrm{MAR})$ ), before gradually declining at similar rates: the oldest age group was around 70 years of age and had average Landolt $C$ acuity of 0.11 $\log$ MAR and average VEP SF limit of $22 \mathrm{cpd}(0.14$ $\log$ MAR) [38].

\section{Effect of stimulus variables on VEP SF limits in normally sighted adults}

\section{a. Temporal frequency}

VEP amplitude is temporally as well as spatially tuned: for fine checkerboards, amplitudes are largest at slow reversal rates ( $\approx 7 \mathrm{~Hz}$ or $14 \mathrm{rps}$ ) but for coarse checkerboards, amplitudes are enhanced at faster reversal rates $(7-11 \mathrm{~Hz}$ or $14-22 \mathrm{rps})$. A similar effect is seen for sinusoidal gratings, tuned at around 5-9 $\mathrm{Hz}$ (10-18 rps) and at around 14-22 Hz (27-44 rps) for fine and coarse gratings, respectively [39]. Despite this, there is broad agreement that VEP SF limits are relatively unaffected by stimulation rates. Similar VEP SF limits were found for $12 \mathrm{rps}$ and $15 \mathrm{rps}$ using reversing sinusoidal gratings and similar contrast and field size, albeit differing mean luminances (40 [20] vs. $50 \mathrm{~cd} \mathrm{~m}^{-2}$ [37]). No difference in extrapolated VEP SF limits were found for reversal rates from 2 to $40 \mathrm{rps}$ (sinusoidal grating, $80 \mathrm{~cd} \mathrm{~m}^{-2}$, circular $4^{\circ}$ ) [23]. Similarly, VEP SF limits for 10 versus $2 \mathrm{rps}$ differed by less than $2.5^{\prime}$ (checkerboard and sinusoidal 


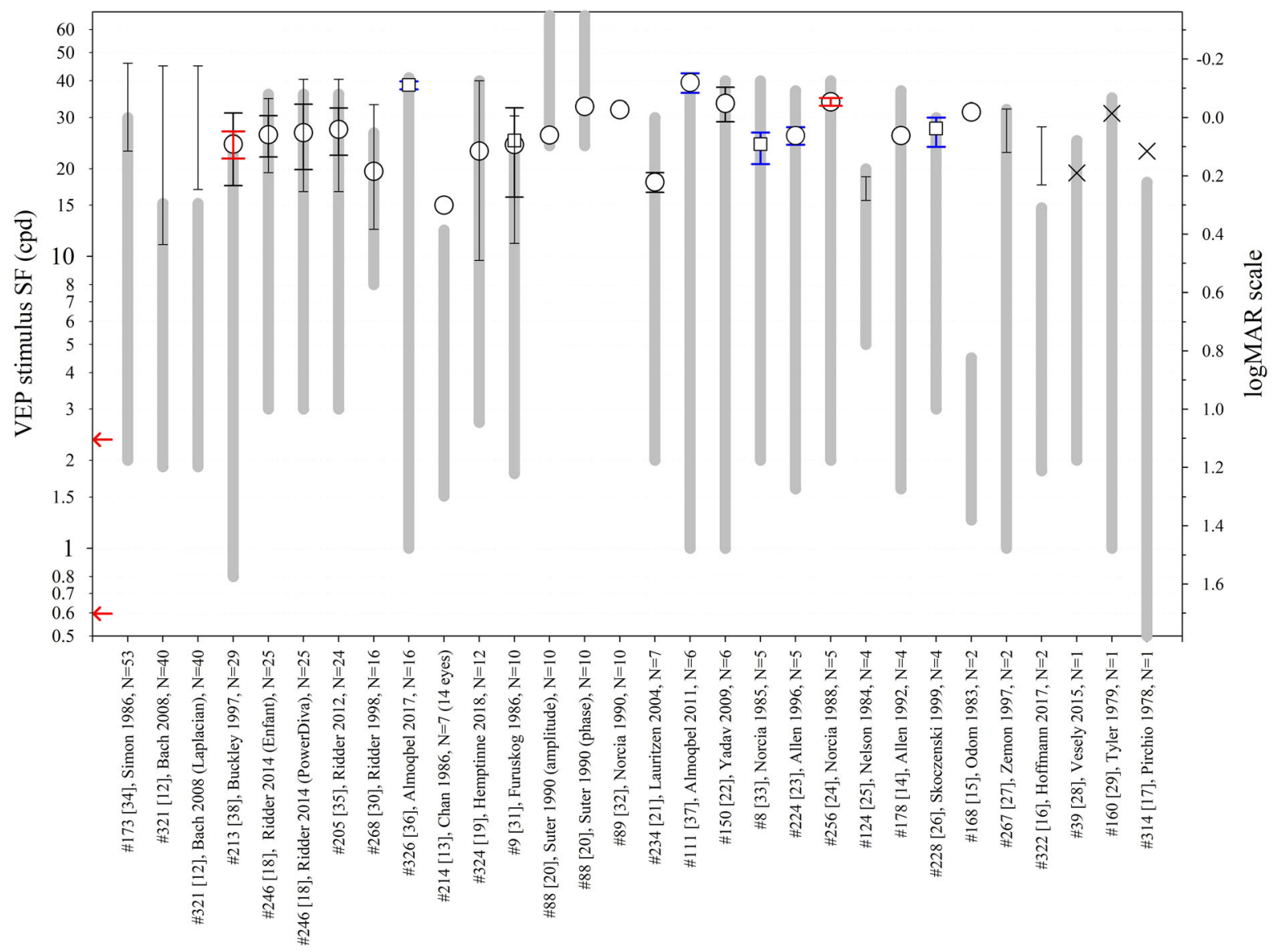

Fig. 2 VEP stimuli SF ranges and limits (cpd) from healthy adults with normal or corrected-to-normal visual acuity as reported in 27 studies. The $\mathrm{x}$-axis lists the publication and the number of subjects. Circles: mean. Squares: geometric mean. $x$ : individual values. Lines with narrow ends: range. Black error

gratings; 15 and $50 \%$ contrast, $50 \mathrm{~cd} \mathrm{~m}^{-2}, 8 \times 11^{\circ}$ ) [40]. Comparing very different reversal rates, 3 versus $43 \mathrm{rps}$, did find a difference with VEP SF limits of 17 versus $10 \mathrm{cpd}$ using a true sweep of sinusoidal gratings (75\% contrast, $\left.50 \mathrm{~cd} \mathrm{~m}^{-2}, 8.25 \times 11.5^{\circ}\right)$ [25]. In terms of success rates (sufficient data for extrapolation), an intermediate reversal rate of $15 \mathrm{rps}$ was slightly better than 12 or 20 rps (reversing sinusoidal gratings, $90 \%$ contrast, $50 \mathrm{~cd} \mathrm{~m}^{-2}$ luminance, $6 \times 6^{\circ}$, $4 \times 4^{\circ}$ and $2 \times 2^{\circ}$ fields) [37]. VEP amplitude at a single checksize $\left(5.5^{\prime}\right)$ was more responsive with acuity, i.e. changed more per acuity-unit-change, for slower ( 3 or $6 \mathrm{rps}$ ) than for faster ( $12 \mathrm{rps}$ ) reversal rates over a small range of near-normal acuities $(80 \%$ contrast, $31 \mathrm{~cd} \mathrm{~m}^{-2}$ luminance, $8.4 \times 6.5^{\circ}$ field) [41]. Finally, in 24 normally sighted adults and 35 bars (wide ends): SD. Red error bars: $95 \%$ confidence interval of mean. Blue error bars: SEM. Thick grey lines indicate the range of spatial frequencies presented or available. Arrows at the SF axis indicate the two ISCEV standard checkwidths, $60^{\prime}$ and $15^{\prime}$ (0.71 and $2.8 \mathrm{cpd} ; 1.63$ and 1.026 in $\log$ MAR units) [66]

amblyopic subjects, VEP SF limits closely matched for on/offset grating stimuli at 3.75 and at $15 \mathrm{~Hz}(80 \%$ contrast, $110 \mathrm{~cd} \mathrm{~m}^{-2}, 12 \times 9^{\circ}$ ), and there were no significant differences in regressions of the VEP SF limits with corresponding psychophysical grating acuities at the two different temporal frequencies (3.75 Hz on/off vs. $15 \mathrm{~Hz}$ on/off) [42].

\section{b. Mean spatial luminance}

Generally, higher luminance stimuli give better VEP SF limits. Increasing luminance from 0.01 through 10 to $100 \mathrm{~cd} \mathrm{~m}^{-2}$ improved VEP SF limits from $\sim 3$ cpd to $\sim 18 \mathrm{cpd}$ to $\sim 26 \mathrm{cpd}$ (sinusoidal gratings, $12 \mathrm{rps}, 80 \%$ contrast) [14]. Increasing mean luminance from 46 to $360 \mathrm{~cd} \mathrm{~m}^{-2}$ improved one subject's VEP SF limit from 11 to $31 \mathrm{cpd}$; however, the luminance 
specificity of this effect is not clear since it was accompanied by four other stimulus changes (reducing field size from $20 \times 15^{\circ}$ to a $2^{\circ}$ diameter disc, increasing the SF available from 12.5 to $35 \mathrm{cpd}$, increasing contrast from 80 to $90 \%$ and changing from sinusoidal to bar gratings) to bring the stimulus closer to the clinical measurement conditions [29]. Smaller changes in luminance at higher mean luminances (25, 50 and Checkerboards and sinusoidal gratings

$100 \mathrm{~cd} \mathrm{~m}^{-2}$ ) did not change VEP SF limits (sinusoidal gratings, $15 \mathrm{rps}, 90 \%$ contrast, $6.3 \times 6^{\circ}$ field) [22].

\section{c. Contrast}

Higher contrast generally results in better VEP SF limits. Increasing contrast from 20 to $100 \%$ improved VEP SF limits from $\sim 11$ to $>20 \mathrm{cpd}$ (checkerboard, $14 \mathrm{rps}, 11 \mathrm{~cd} \mathrm{~m}^{-2}, 4.5^{\circ}$ diameter field) [43]. Similarly, the low amplitude VEPs found in around $10 \%$ of healthy subjects could be induced to match amplitudes of the rest of the group by increasing contrast from 40 to $80 \%$ (16 Hz on/offset sinusoidal gratings, $17 \mathrm{~cd} \mathrm{~m}^{-2}, 5^{\circ}$ diameter field [44]. VEP SF limits systematically improved by up to $4.3^{\prime}$ for $50 \%$ versus $15 \%$ contrast (checkerboard and sinusoidal gratings; two and $10 \mathrm{rps}, 50 \mathrm{~cd} \mathrm{~m}^{-2}, 8 \times 11^{\circ}$ field size) [40].

Higher contrast does have the disadvantage of causing one or more notches-reduced amplitude VEPs at intermediate SFs - in the SF tuning curve which could cause a marked underestimate of VEP SF limit [45-47]. This notch was evident for contrasts greater than $\sim 40 \%$ (reversing sinusoidal gratings, $16-48 \mathrm{rps}, 40 \mathrm{~cd} \mathrm{~m}^{-2}, 10 \times 12^{\circ}$ field), although the authors noted that high contrast $(>50 \%)$ increased SNR [48] and other workers noted a notch even at contrasts lower than $40 \%$ [49]. Lower contrast has another potential benefit as a narrower luminance range is sufficient, which is less sensitive to inaccuracies of gamma correction and thus helps avoid luminance artefacts [12]. Healthy adult viewers at least tend to find lower contrast stimuli more comfortable to watch [19].

\section{d. Field size}

As a rule of thumb, finer patterns $\left(<15^{\prime}\right.$ element width) evoke mainly foveal VEPs, whereas coarser patterns ( $>30^{\prime}$ element width) evoke VEPs also via extrafoveal stimulation [39]. VEP amplitudes increase with increasing field size ( $2-9^{\circ}$ diameter), and more so for coarser patterns; for smaller patterns $\left(\leq 5^{\prime}\right)$, field sizes $>4^{\circ}$ diameter do not cause increased VEP amplitude (reversing checkerboard, 14 rps, $310 \mathrm{~cd} \mathrm{~m}^{-2}, 95 \%$ contrast [50]. Circular field sizes of $15-4^{\circ}$ produced similar VEP SF limits of around $11 \mathrm{cpd}$ despite generally reducing VEP amplitudes, although amplitudes to a $2^{\circ}$ field were too low for extrapolation (reversing gratings, $24 \mathrm{rps}, 80 \%$ contrast, $46 \mathrm{~cd} \mathrm{~m}^{-2}$ luminance) [29]. Similarly, field sizes of $6 \times 6^{\circ}, 4 \times 4^{\circ}$ and $2 \times 2^{\circ}$ did not produce different VEP SF limits (reversing sinusoidal gratings, 12 and $15 \mathrm{rps}, 90 \%$ contrast, $50 \mathrm{~cd} \mathrm{~m}^{-2}$ luminance) [37]. Similarly, although VEP magnitudes were generally larger for one VEP system with a large $\left(13 \times 10^{\circ}\right)$ field than for another using a smaller field $\left(3 \times 6^{\circ}\right)$, the VEP SF limits did not differ. Both systems employed sinusoidal gratings of $100 \mathrm{~cd} \mathrm{~m}^{-2}$ and $80 \%$ contrast reversing at $15 \mathrm{rps}$, and only slightly different sets of SFs [18].

\section{e. Pattern}

Checkerboards and sinusoidal gratings are most commonly used, with square-wave gratings (bars) also described. Surprisingly, no studies have compared VEP SF limits from such stimuli. Sinusoidal gratings are simpler stimuli in that they contain a single SF and obviate the need to interpret pattern element size [40]. The sharp edges of square-wave gratings or checkerboards may provide a better accommodative stimulus [27]. No systematic VEP SF limit differences were found between horizontally and vertically oriented gratings $[25,48]$ although an oblique effect is evident, with poorer VEP SF limits for oblique than for orthogonal orientations [25]. Since a checkerboard's $\mathrm{SF}_{\mathrm{f}}$ is oriented obliquely $[9,10]$, this finding may have relevance for checkerboard VEP SF limits.

\section{f. Reversal versus on/offset modulation}

Pattern reversal, or counterphase modulation, maintains constant mean luminance and only contrast alters. Two reversals comprise one cycle, thus 16 rps $=8 \mathrm{~Hz}$. The neural response to each reversal is the same, and therefore, the electroencephalogram (EEG) spectrum has only even harmonics of the stimulus frequency $(1 / 2 \mathrm{rps})$ [51]. For clarity, the frequency of a pattern-reversal stimulus should be specified in rps to 
avoid any confusion over whether a frequency in $\mathrm{Hz}$ incorrectly refers to reversal rate or correctly refers to the stimulus frequency, i.e. two reversals. Pattern on/ offset modulation periodically exchanges the pattern with an isoluminant grey field: differing neural responses to the onset and offset of the pattern and/ or differing onset and offset durations create both even and odd harmonics in the EEG spectrum. Luminance artefacts are possible if the grey field is not matched in average spatial luminance to the patterned field.

A more marked notch at intermediate SFs was found for reversing stimuli than for on/offset stimuli $[44,52]$. Strasburger and co-workers also noted that on-off modulation reflects stimulus visibility at high SF more accurately than reversal [44]. Brief onsets (e.g. $40 \mathrm{~ms}$ ) cause the on- and off-responses to overlap, producing a larger and therefore more detectable VEP than longer onsets (e.g. $300 \mathrm{~ms}$ ) [53, 54]. One study compared VEP SF limits to $15 \mathrm{~Hz}$ on/offset and $15 \mathrm{rps}$ reversing stimuli in 22 normally sighted adults and 31 adults with amblyopia $\left(80 \%\right.$ contrast, $\left.110 \mathrm{~cd} \mathrm{~m}^{-2}, 12 \times 9^{\circ}\right)$. Their VEP SF limits were highly correlated $(r=0.79)$, and there were no significant differences in regressions of the VEP SF limits with corresponding psychophysical grating acuities at the two different pattern presentations [42].

\section{g. Spatial frequency (SF) properties}

SF properties include sampling range, sampling density, direction of SF change and adaptation effects. The validity of any extrapolation technique depends on adequately dense and extensive sampling of the VEP amplitude versus SF function, especially with reversing stimuli which may produce multiple peaks, i.e. a notched function. In healthy individuals at least, patterns up to $40 \mathrm{cpd}$ may be required: an $8 \mathrm{cpd}$ upper limit was not sufficiently high to avoid underestimation errors [33]. Even with an upper limit of $27 \mathrm{cpd}$, a ceiling effect was noted: it has been suggested that the SF range should bracket a subject's VEP SF limit $[30,55]$.

Linear sampling ${ }^{1}$ of SF (equal spacing of stimuli SFs (cpd) on a linear scale) results in desirably fine

\footnotetext{
${ }^{1}$ Note this section discusses linear versus exponential sampling of SF. The separate, but related, issue of linear versus logarithmic SF axis scaling is discussed.
}

sampling towards the VEP SF limit of normal adults, thus accurately representing the final slope. However, discrete steps in pixel size result in nonlinear changes in element area at the finest SFs, e.g. $1 \times 1$ to $2 \times 2$ to $3 \times 3$ pixels. Exponential sampling (equal spacing of stimuli SFs (cpd) on a logarithmic scale) gives equal weight to each octave of SF (Fig. 3), and results in a VEP amplitude versus SF function which corresponds with psychophysical tuning functions [56, 57]. However, spatial resolution reduces towards the acuity limit and some authors therefore describe exponential SF sampling as unsuitable for acuity measurement [29, 33]. To our knowledge, no direct comparison of exponential versus linear SF sampling has been undertaken.

The presence of SF 'channels' (neural populations selectively sensitive to limited but overlapping SFs taking hundreds of milliseconds to reach steady state [56, 58-60]) theoretically advocates for sequential SF presentation. Random SF sampling could stimulate a different spatial 'channel' with each change in SF, increasing the number of times steady state must be reached and potentially lengthening test time [33]. VEP amplitude stabilises several seconds after stimulus onset [61, 62]); however, no effect on VEP SF limit was found for stimulus durations from 1 to $8 \mathrm{~s}$ per stimulus [30], nor for stepwise sweeps varying in duration from 11 to $20 \mathrm{~s}$ [37].

The direction of SF change (coarse-to-fine or fineto-coarse) does not appear to incur significant VEP hysteresis, i.e. minimal adaptation effect [63], perhaps due to the relatively narrow bandwidth of SF channels ( $\approx$ 1-1.4 octaves) [64] or the multiple SFs present in bar gratings and checkerboards. Studies comparing VEP SF limits obtained using coarse-to-fine and fineto-coarse SF changes found no differences [19, 37, 65] although subjects were noted to be more attentive to coarse-to-fine stepwise sweeps [65].

In summary, suitable stimuli for VEP SF limit measurements in healthy adults have stimulation rates in the range of $\approx 10-24 \mathrm{rps}$ for reversing stimuli or 5-12 Hz for on/offset stimuli. A large range of mean luminances have been successfully used, including values in the range of around $25-100 \mathrm{~cd} \mathrm{~m}^{-2}$. Contrast choice should balance the requirement for better SNR with any need to avoid a notch (reversing patterns), to avoid a luminance artefact (on/offset patterns), and to ensure viewing comfort. Field sizes $>2^{\circ}$ are suitable. There are few data to guide 


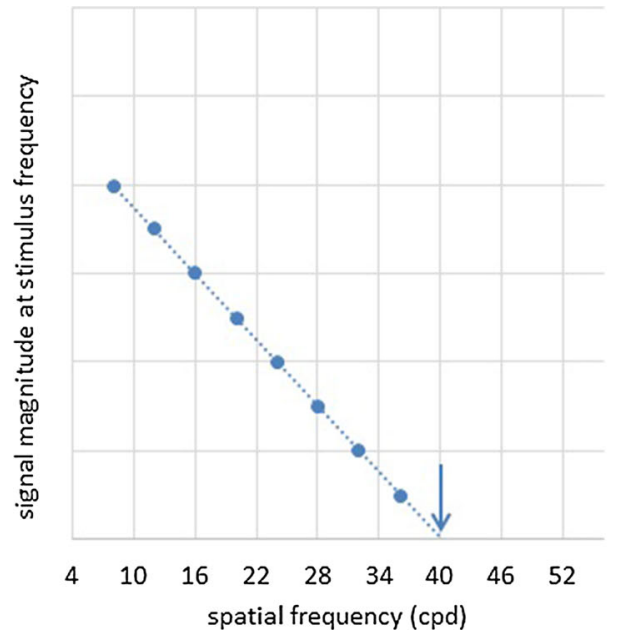

Fig. 3 Effect of sampling-illustration of VEP SF limits for artificial data sampled linearly (left) and exponentially (right) with SF. Data show a linear relationship between VEP magnitude and SF close to threshold using linear axis scales.

the choice between sinusoidal gratings, square-wave gratings or checkerboards; grating orientation (horizontal/vertical) does not appear to affect VEP SF limits, but as with subjective thresholds, there is a small oblique effect. On/offset modulation produces VEPs which are consistently larger across all SFs than reversal modulation, but greater care is required to avoid luminance artefacts. Brief onset durations offer further amplitude enhancement over longer onset duration. The range of SFs should approach or bracket subjects' limits for acceptable accuracy and should include sufficient data points for acceptable precision of VEP SF limit. Linear SF sampling benefits accuracy due to higher sampling close to threshold, while exponential SF sampling more closely emulates psychophysical tuning functions. Neither stimulus duration per SF condition (1-8 s), sweep duration (11-20 s) nor direction of SF change (coarse-to-fine or fine-to-coarse) appear to alter significantly VEP SF limits.

\section{Effect of acquisition and analysis variables} on normally sighted adult VEP SF limits

\section{a. Monocular versus binocular viewing}

Specific clinical requirements determine whether VEP SF limits should be recorded monocularly or binocularly; studies of healthy adults have used either

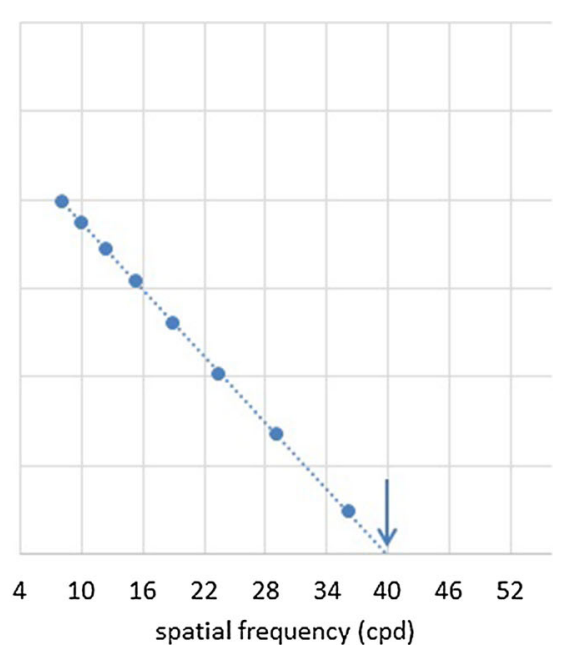

Linear SF sampling (left) improves sampling density close to typical adult VEP SF limit, while exponential SF sampling (right) maps more closely to psychophysical SF channels

monocular or binocular viewing in approximately equal number. Limited data comparing the two viewing conditions suggest binocular VEP amplitude versus SF functions have amplitudes larger than those of monocular functions by a factor of around 1-2, but VEP SF limits are similar [48].

b. Electrode position and montage

ISCEV standard VEPs [66] require an active electrode at the midline over the occiput $(\mathrm{Oz})$ referred to a distant, frontal electrode $(\mathrm{Fz})$. Alternative lateral active electrode sites (PO7, O1, O2, PO8) produced similar VEP SF limits to $\mathrm{Oz}$, but tended to have lower SNR and thus fewer subjects had viable threshold extrapolations [22, 37]. Averaging viable limits from five occipital channels resulted in the lowest coefficient of variation compared with using selected channels [21]. A 64-channel study using the average of all channels as the reference found $\mathrm{Oz}$ to be the optimal active electrode site, but noted the most sensitive zone stretches down towards the inion (Iz) and laterally to PO7, O1, O2 and PO8. Coarse patterns were often detected over right occipital/parietal positions, while fine patterns were optimally detected at midline positions [19].

VEPs are not necessarily symmetric about the midline in many individuals. Selecting data from one of two occipital channels with closely spaced active and reference electrodes $(\mathrm{Oz}-\mathrm{O} 1$ and $\mathrm{Oz}-\mathrm{O} 2)$ based 
on highest SNR at peak VEP magnitude might optimise detection of an individual's VEP SF limit [23, 24, 26, 32, 65]. This principle is extended via Laplacian electrode montages which localise evoked potential sources and improve VEP SNR using closely placed active and reference electrodes, as each carries highly coherent noise [67]. This produces good cancellation of remote noise such as eye movements or spatially diffuse noise such as EEG alpha activity. The active site, usually close to Oz for VEPs, uses the arithmetic mean of voltages from the surrounding electrodes as its effective reference voltage. For example, a Laplacian channel using a montage of $\mathrm{Oz}$ (active) and lateral electrodes at $\mathrm{O} 1$ and at $\mathrm{O} 2$ would use $\mathrm{Oz}$ voltage at the positive/active input and $(1 / 2(\mathrm{O} 1+\mathrm{O} 2))$ voltage at the negative/reference input. Optimal lateral electrode positions are at $15 \%$ of half-head circumference $(4-4.5 \mathrm{~cm}$ for normal adults) [68]. This one-dimensional Laplacian montage was faster than an Oz-Fz montage at detecting VEPs to fine patterns, e.g. those close to VEP SF limit [68]. The same Laplacian montage found similar VEP SF limits as an Oz-Fz montage, but with lower variability $[12,69]$. A two-dimensional Laplacian montage (four electrodes placed orthogonally $3 \mathrm{~cm}$ around an active electrode site $2 \mathrm{~cm}$ above $\mathrm{Iz}$ ) also enhanced SNR and improved intra-subject reliability, while eliciting comparable VEP SF limits to a traditional montage ( $2 \mathrm{~cm}$ above Iz referred to Fz) [70]. Thus, the key benefit of a Laplacian montage is enhanced SNR, especially close to threshold.

\section{c. Criteria for VEP detection}

Steady-state VEP analysis in the frequency domain, sometimes following time domain averaging, typically uses only the first harmonic (at the stimulus frequency) for pattern on/offset stimuli or second harmonic (at the reversal rate) for pattern-reversal stimuli. Including one [26, 71-77] or even more $[78,79]$ higher harmonics has been explored. Only one study combined harmonics for analysis, using a simple sum of the first and second harmonic magnitudes [76]. In other steady-state VEP applications, the square root of summed harmonic powers has been used to combine harmonics for a "global SNR" [80]. While this assumes that harmonics reflect a common response [81], which may not necessarily be the case [82], using a global SNR to improve diagnostic utility of VEP SF limits rather than to probe pathophysiological processes may be justified.

Signal detection commonly includes a criterion of $\mathrm{SNR} \geq 3[14,18,21-23,26,28,32,33,37,65,83,84]$ with noise defined by an adjacent frequency bin $[32,33,84]$ or mean of the two adjacent bins [21-23, 26, 65]. Absence of large artefacts at "noise" frequencies has often been used as an additional criterion for accepting presence of a VEP [14, 33, 65]. Criteria based on absolute amplitude or magnitude (e.g. $1 \mu \mathrm{V})$ are not reliable because of high interindividual variability in noise and in VEP magnitude [85]. A SNR of 3, based on noise magnitude at one adjacent frequency bin, is associated with an empirical false "positive" rate of $0.3 \%$ [33], i.e. a 1-in-333 chance of incorrectly declaring noise to be a VEP. Conversely, a SNR of 3, based on noise as mean of magnitudes at two adjacent bins, has a $4.1 \%$ empirical false positive rate [85], i.e. a 1-in-25 chance of incorrectly declaring a VEP to be noise. A SNR of at least 3 therefore appears to represent a suitable criterion with acceptable sensitivity and specificity.

Unless the DFT output at the stimulus frequency is adjusted for noise estimates, SNR is more correctly signal-plus-noise to noise ratio because the signal's frequency bin also includes noise, i.e. non-visually driven EEG occurring at the stimulus frequency $[12,85]$. An alternative criterion requires that the 95\% confidence interval of magnitudes, calculated from DFTs of several EEG epochs, should exclude zero: it is not stated whether the signal magnitude measures are noise-corrected (Enfant ${ }^{\circledR}$ proprietary technique, Diopsys Inc., Pine Brook, NJ, USA [18]).

Neither SNR nor magnitude criteria use the phase data produced by Fourier analysis. Phase tends to increase (lag) gradually across coarser SFs and then lag more steeply across higher SF stimuli. It may show large shifts at mid-range SFs, particularly if there is a "notch". Phase coherence is better to supra-threshold gratings, while noise is characterised by highly incoherent phase [30, 49, 86, 87]. These characteristics have been employed for signal detection alongside SNR criteria, either by requiring physiologically plausible phase lead or lag with decreasing or increasing SF respectively $[14,21,22,26,28,32$, $33,88]$, or by requiring that the $95 \%$ confidence interval of phase exceeds an empirical criterion of $90^{\circ}$ $[30,55]$. 
Fourier analysis produces bivariate data (phase and amplitude); the sine and cosine coefficients can be used to create a complex plane vector for each EEG epoch, with vector length representing magnitude and angle representing phase. Hotelling's $t^{2}$ statistic [89] and the more powerful circular $\mathrm{T}^{2}$ statistic [90] assume a VEP to be present if the elliptical or circular $95 \%$ confidence intervals constructed around the vector tips exclude the origin. The circular $\mathrm{T}^{2}$ statistic assumes equal variances for the real and imaginary vector components (hence "circular") and is equivalent to the magnitude-squared coherence statistic [91]. The criterion of $95 \%$ confidence interval excluding the origin is identical to a SNR $>1$, where signal is defined as mean vector length (VEP magnitude) and noise is defined as radius of the confidence interval [18, 27, 92].

Comparing vectors from stimulated EEG segments with no-stimulus vectors improved VEP SF limits a little compared with a magnitude-only criterion (26.4 cpd vs. $25.4 \mathrm{cpd}$ ). A phase-stability criterion produced even better VEP SF limits (30.3 cpd) [20]. These analyses can be used on raw (non-averaged) data, affording real-time analysis [92]. Statistics which use both magnitude and phase outperform those using only one [91]. However, in real-time analyses, different statistics can be complementary. A SNR criterion detected supra-threshold VEPs sooner than the circular $\mathrm{T}^{2}$ statistic because it can be applied as soon as the first EEG epoch is acquired, while the circular $\mathrm{T}^{2}$ statistic cannot be applied until three EEG epochs are available. Conversely, in low SNR conditions, the circular $\mathrm{T}^{2}$ statistic is more powerful and detected VEPs close to threshold faster than the SNR criterion [93]. With suitable adjustment for multiple tests, SNR and circular $\mathrm{T}^{2}$ statistic can be used simultaneously to minimise the duration of VEP SF limit testing in a real-time system designed for assessing paediatric patients [92, 94, 95].

For techniques using VEPs (transient or steady state) analysed in the time domain, presence or absence of a VEP is determined subjectively by eye [40, 41, 53, 54, 69, 70, 87, 96-102], sometimes with an additional requirement of a criterion amplitude for P100 [100, 103]. Time domain, objective methods [104-106], which can be employed adaptively in real time to shorten recordings to the minimum necessary for an objective quality measure of the averaged response [107], are unfortunately seldom used for transient VEPs.

\section{d. Definition of threshold}

The majority of studies define the VEP SF limit by extrapolating a straight line regressed through significant VEP amplitudes or magnitudes plotted versus SF to $0 \mu \mathrm{V}$ or to another floor such as a noise estimate. The commonly used linear extrapolation to $0 \mu \mathrm{V}$ approach aimed to minimise bias since the VEP is likely to still be present, but below noise amplitude, at the SFs closest to threshold. It assumes that the function which holds for supra-noise VEPs will also hold for sub-noise VEPs [29]. A few studies define the VEP SF limit by curvilinear fitting, e.g. parabolic, modified Ricker or other curves [78, 98, 102, 108, 109] to magnitude or amplitude data plotted versus SF. These functions are commonly fitted to plots where a linear scale has been used for SF, although logarithmic scales have also been used (Fig. 4) [12, 15-17, 31, 40, 54, 110-113]. A linear SF scale is justified since log contrast sensitivity drops linearly with SF at high SF ( $>5 \mathrm{cpd}$ ) [114] and VEP amplitude drops linearly with $\log$ contrast close to threshold $[6,115]$. VEP amplitude therefore theoretically drops linearly with linear SF close to threshold [33]. A linear-linear relationship has been demonstrated in adults [29, 116] and linear extrapolation to zero microvolts on a linear SF axis is insensitive to VEP amplitude changes [117]; logarithmic SF scaling potentially introduces a systematic error, skewing the linear regression to "better" thresholds-the greater the number of points away from threshold used, the greater the skew (Fig. 4).

Certainly, better thresholds with logarithmically scaled SF were found in the two studies which compared VEP SF limits with linear and with logarithmic SF scaling of the same data. Mean VEP SF limits from fourteen eyes of seven healthy individuals were better, but more variable, for logarithmic SF scaling (37 cpd, range 29-73 cpd) than for linear SF scaling of the same data (30 cpd, range $26-41 \mathrm{cpd}$ ) [13]. A study of 21 normally sighted adults and older children also found better VEP SF limits for logarithmically scaled SF (median $16 \mathrm{cpd}$, range 5-243 cpd) than for linearly scaled SF (median $11 \mathrm{cpd}$, range 4-30 cpd) [84]. Each study found logarithmically scaled SFs produced two cases with unrealistically 


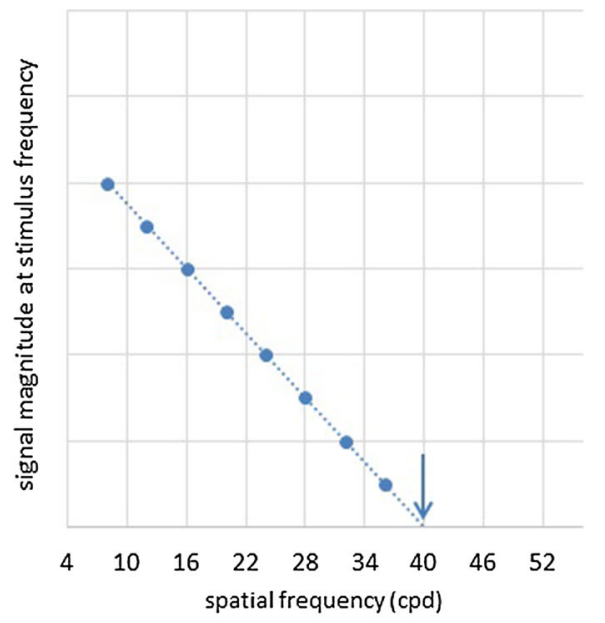

Fig. 4 Effect of scaling-illustration of VEP SF limits for artificial data plotted versus linear (left) and versus logarithmic (right) SF. Linear extrapolation to $0 \mu \mathrm{V}$ with a linear SF axis scale (left) gives a VEP SF limit of $40 \mathrm{cpd}$ (1.6 log cpd) (solid arrows). Linear extrapolation of the same data to $0 \mu \mathrm{V}$ using a logarithmic SF axis scale (right) gives a "better" VEP SF limit

good limits, e.g. 67 and 73 cpd [13] and 158 and 243 cpd [84]. Both studies used a limited range of SFs with the finest SFs being only 10 or $12 \mathrm{cpd}$ : the larger the gap between the highest SF available and the individual's VEP SF limit, the larger the error in the extrapolated limit. The error is much larger, and skewed to unrealistically good limits, when logarithmic SF scaling is used.

For the extrapolation limit, multiple criteria have been employed to define threshold, most commonly $0 \mu \mathrm{V}$ or some estimate of noise floor. Studies comparing the effect of absolute floor levels $(0,1$ and $2 \mu \mathrm{V}$ ) found, as might be expected, that VEP SF limits worsened by at least $5 \mathrm{cpd}$ with each $1 \mu \mathrm{V}$ increase in noise criteria $[55,118]$. The $0 \mu \mathrm{V}$ criterion is widely used, perhaps originating from early experiments where extensive time domain averaging of ssVEPs reduced noise to negligible levels [20], but also theoretically justified since at the point of absent cortical signal, i.e. acuity limit, neuronal noise (as opposed to EEG noise) is low. However, some of the magnitude output of a DFT at the stimulus frequency is due to non-visually driven EEG at that frequency, and hence is noise [85]: the relative proportion which is noise increases in small VEP signal conditions, e.g. close to threshold. Therefore, "raw" magnitudes extrapolated to $0 \mu \mathrm{V}$ will overestimate VEP SF limits compared with noise-corrected magnitude estimates

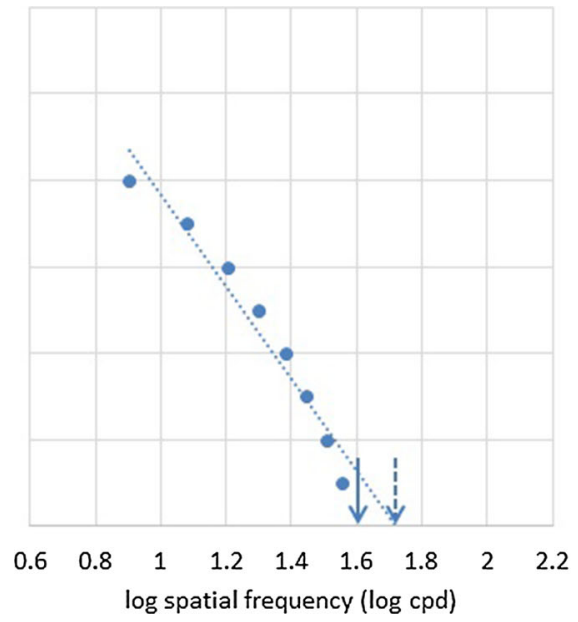

of $50 \mathrm{cpd}(1.7 \log \mathrm{cpd})$ (dashed arrow). If the true relationship between VEP amplitude and linear SF close to threshold is linear as shown, then logarithmic SF scaling with linear regression may introduce a systematic error giving erroneously "better" VEP SF limits

$[12,25,44]$. This overestimation is likely to be small for signals with good SNR: Norcia et al. note that threshold estimates based on data points with SNR > 3 are "virtually uncontaminated" by EEG noise $[33,85]$ : at a noise-corrected SNR of 3 , there is little $(5.3 \%)$ noise [85]. If noise estimates are not discarded before extrapolation [85], any overestimation relative to noise-corrected amplitudes could be reduced by extrapolating to a noise floor rather than to zero [20, 27].

An alternative strategy defines VEP SF limit as the finest SF evoking a significant VEP, which ought to underestimate thresholds found by extrapolation. A direct comparison of the two techniques in adults does indeed show an underestimation of $0.5-1$ octave (0.15-0.3 log units) [38], or $0.25-0.5$ octaves (0.08-0.15 log units) [33], but the underestimate depends strongly on SF sampling density close to threshold. Adult thresholds using the finest SF technique do not differ markedly from other studies reporting thresholds based on extrapolation (see Fig. 2): for example, two studies using the finest SF technique found VEP SF limits of 9.7-40 cpd [19] and 9.4-24 cpd [92], similar to those typically found for extrapolation techniques. No study has compared the two techniques in the same subjects, although retrospective analysis would be straightforward. Since the finest SF technique does not require SF sampling 
density sufficient to characterise a major portion of the VEP magnitude versus SF function, there is potential for defining limits faster by concentrating recording close to threshold [92].

Extrapolation techniques can fail to define a VEP SF limit, usually because the final, descending limb of the magnitude versus SF function is poorly defined due to deep notches or generally low amplitudes. These failures occurred on 29/384 individual sweeps (8\%) [30] and in 2/108 recordings (2\%) [12]. One approach under such circumstances is to include the finest $\mathrm{SF}$ technique as an additional, integrated strategy [18, 30, 35].

Data acquisition which is time-locked to the stimulus avoids artefacts (overspill or leakage into nearby frequency bins) from the Fourier analysis, thus maximising SNR [27, 119]. This can be achieved by appropriate selection of EEG epoch (i.e. sweep duration or analysis period) as an integer multiple of stimulus periods. For example, an $8.0 \mathrm{~Hz}$ stimulus has a period of $125 \mathrm{~ms}$ : EEG epochs for analysis should be some integer multiple such as $8 \times 125 \mathrm{~ms}$, i.e. $1000 \mathrm{~ms}$. If stimulus and acquisition are not appropriately time-locked, then frequency domain artefacts can be reduced, but not eliminated, by application of windowing techniques. Artefacts can be eliminated by truncating the analysis interval to encompass an integer number of stimulus cycles [119].

In summary, active electrodes close to $\mathrm{Oz}$ are sited to define VEP SF limits, and closely positioned reference electrodes, especially in a Laplacian montage, enhance SNR towards threshold. Frequency domain analysis, usually via a DFT, can be subjected to statistical analyses to determine the likelihood of a signal at the stimulus frequency being noise: these statistics can employ magnitude-only measures such as SNR, or include additional phase criteria, or combine magnitude and phase (e.g. circular $\mathrm{T}^{2}$ ). VEP SF limits can be defined by linear (or other functions) extrapolation of significant (non-noise) VEPs plotted versus linearly or logarithmically scaled SF: logarithmic SF scaling may introduce a systematic error, skewing the linear regression to better thresholds. The intercept with $0 \mu \mathrm{V}$ is commonly used to define the VEP SF limit; this may result in slightly better thresholds for "raw" VEP magnitudes compared with noise-corrected VEP magnitudes. Alternative noise floors based on measured levels avoids this overestimation for non-noise-corrected DFT magnitudes. Extrapolation techniques occasionally fail to define a VEP SF limit. An alternative, and possibly faster, approach to extrapolation is to use the finest SF criteria to define VEP SF limit. Optimal, artefact-free EEG spectra can be ensured by using an EEG sampling rate which is an integer multiple of the monitor's frame rate, if relevant, and by choosing or truncating the analysis period to be an integer multiple of the stimulus period.

\section{Correspondence of VEP SF limits}

with behavioural thresholds in normally sighted adults

In some of the work already described, an implicit or explicit aim was to develop a VEP technique whose SF limit agreed with perceptual thresholds. Often, close agreement was taken as an indication of the quality of the VEP technique, even though an exact match would be surprising given the multiple different mechanisms involved, listed in the introduction. Agreement has sometimes been "improved" using techniques which may have some systematic error or bias, for example logarithmic SF scaling or using a $0 \mu \mathrm{V}$ intercept for non-noise-corrected magnitudes. The aim of this section is to describe disparities between VEP SF limits and perceptual SF thresholds under three circumstances: studies employing identical stimuli; studies comparing VEP SF limits with behavioural acuity tests using discrimination tasks; studies comparing VEP SF limits with behavioural acuity tests using identification tasks.

\section{Identical stimuli}

We identified nine studies where the same, normally sighted adult subjects had psychophysical acuity and VEP SF limits assessed using identical stimuli (Table 2, Fig. 5a). Seven found poorer VEP SF limits than psychophysical acuity. An early paper recorded three thresholds to a red and black reversing checkerboard by changing the viewing distance: subjects could perceive apparent motion at an average distance of $4.2 \mathrm{~m}$ ( $\approx 10.2 \mathrm{cpd}$ ), could perceive stationary checkerboards at $2.9 \mathrm{~m}(\approx 7.05 \mathrm{cpd})$ and could evoke measurable VEPs at around $1.3 \mathrm{~m}(\approx 3.2 \mathrm{cpd})$ [105]. VEP SF limits in four adults were about $25 \mathrm{cpd}$, while psychophysical thresholds, based on two-alternative forced-choice technique, were about $50 \mathrm{cpd}$ for high- 


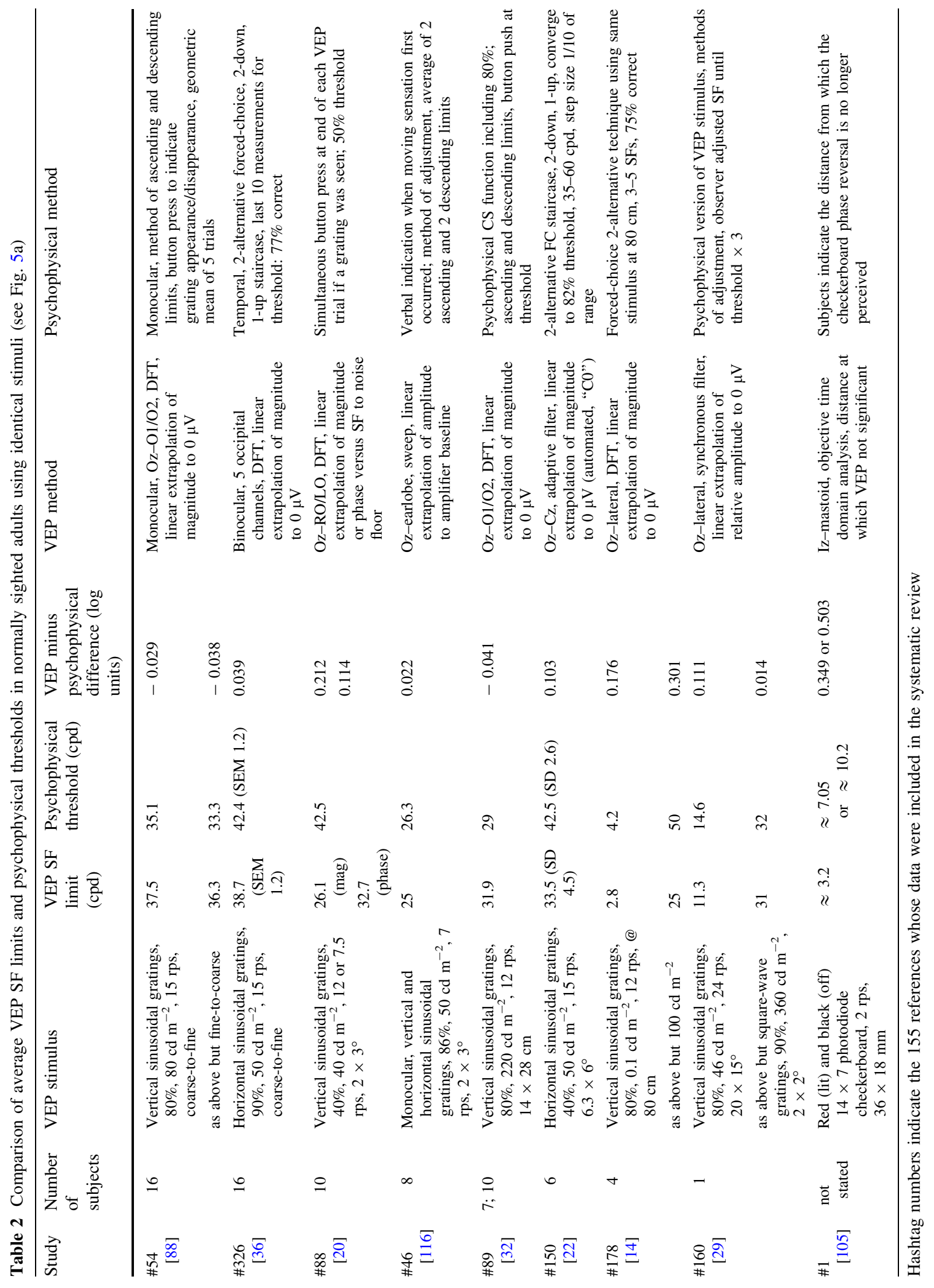


(a)

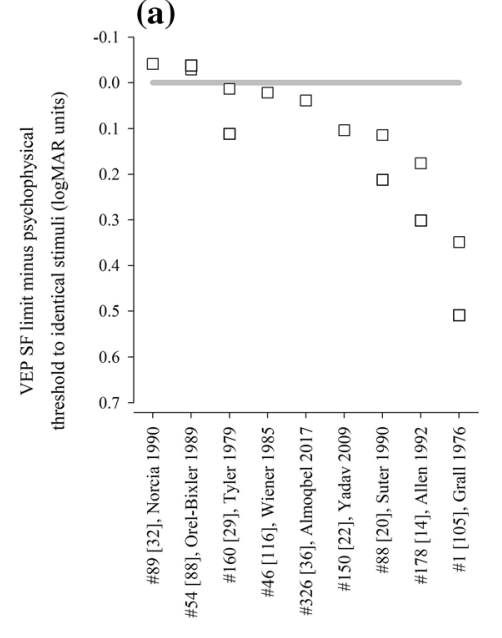

(b)

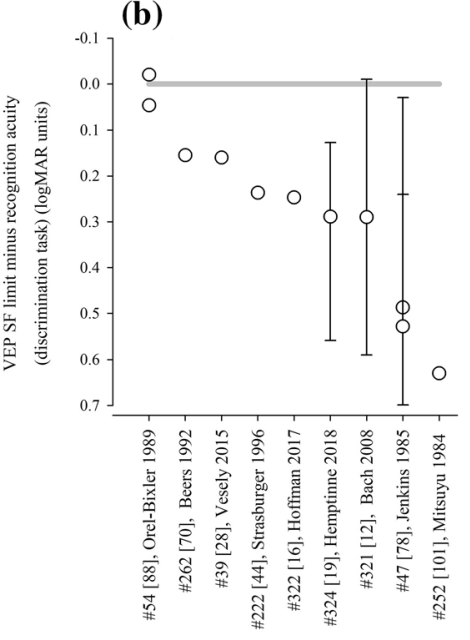

(c)

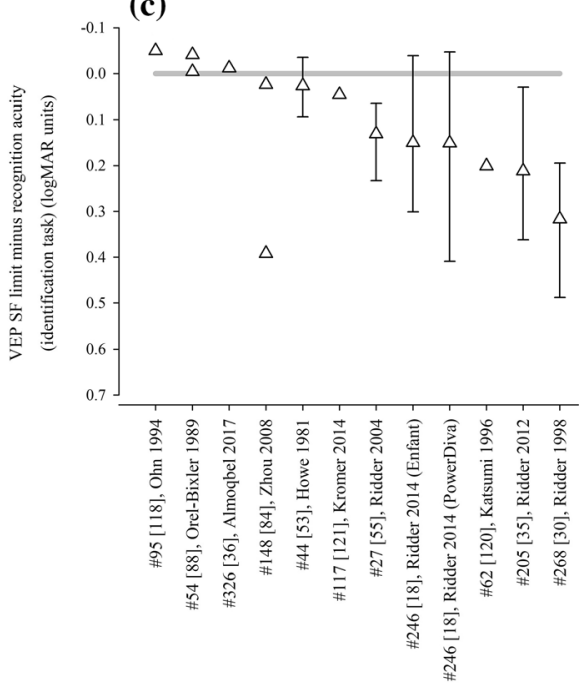

Fig. 5 Illustration of studies reporting differences between VEP SF limits and behavioural thresholds in healthy adults using a psychophysical testing with VEP stimuli (Table 2); b recognition acuity based on a discrimination task (Table 3 ) and $\mathbf{c}$ recognition acuity based on an identification task

luminance $\left(100 \mathrm{~cd} \mathrm{~s} / \mathrm{m}^{2}\right)$ gratings-the difference lessened for dimmer gratings [14]. Based on a button press at the end of each VEP trial if a grating had been seen, psychophysical thresholds (42.5 cpd) exceeded VEP SF limits using either magnitude $(26.1 \mathrm{cpd})$ or phase (32.7 cpd) criteria [20]. Similarly, psychophysical thresholds were 42.5 and $42.4 \mathrm{cpd}$ on average compared with mean VEP SF limits of around 33.5 and $38.7 \mathrm{cpd}$ [22, 36]. Sweep VEP SF limits to reversing sinewave gratings were $25 \mathrm{cpd}$ on average, slightly poorer than psychophysical thresholds of $26.3 \mathrm{cpd}$ [116]. In a study of a single adult, an 11.3 cpd VEP SF limit and a 14.6 cpd psychophysical threshold improved to $31 \mathrm{cpd}$ and $32 \mathrm{cpd}$, respectively, by increasing mean luminance from 46 to $360 \mathrm{~cd} \mathrm{~m}^{-2}$, reducing field size from $20 \times 15^{\circ}$ to $2 \times 2^{\circ}$ and increasing contrast from 80 to $90 \%$ [29]; Nelson et al. subsequently noted of this study that VEP SF limits averaged $85 \%$ of psychophysical acuity limits [25]. In two studies using very similar methodologies, the reverse situation was found: VEP SF limits were slightly better than psychophysical thresholds (31.9 vs. 29.0 cpd [32], 37.5 vs. $35.1 \mathrm{cpd}$ [88]).

These data suggest that VEP SF limits are per se different to, and probably slightly poorer than, behavioural or psychophysical thresholds to identical stimuli. The gap is very variable across the studies
(Table 4). Grey lines indicate no difference: points below the line indicate VEP SF limits are poorer than behavioural thresholds. Error bars indicate 95\% limits of agreement (not always available)

reviewed and depends strongly on the stimulus, with high contrast, high-luminance stimuli generally producing closer agreement than for lower contrast, lower luminance stimuli (Fig. 5a).

\section{Non-identical stimuli-recognition (discrimination) acuity tests}

We identified nine studies where the normally sighted adult subjects had both VEP SF limits and a discrimination acuity measured, i.e. tumbling $\mathrm{E}$ or Landolt $\mathrm{C}$ charts (Table 3, Fig. 5b). Studies were included if sufficient detail was available to describe both average VEP SF limits and acuities: when possible, variability and limits of agreement were also extracted. Often, these findings were part of a study which included a spectrum of visual abilities, with poorer vision recorded from patients or from artificially impaired adults: only data from healthy, optimally refracted adults are presented in this section. Acuities were converted from native units into $\log$ MAR units as necessary, and VEP SF limits (cpd) were also expressed in logMAR units to allow comparison with acuity (Table 1).

Average acuities were better than VEP SF limits in most cases. Extrapolating findings for nine subjects performing at the $0.000 \log$ MAR level showed 


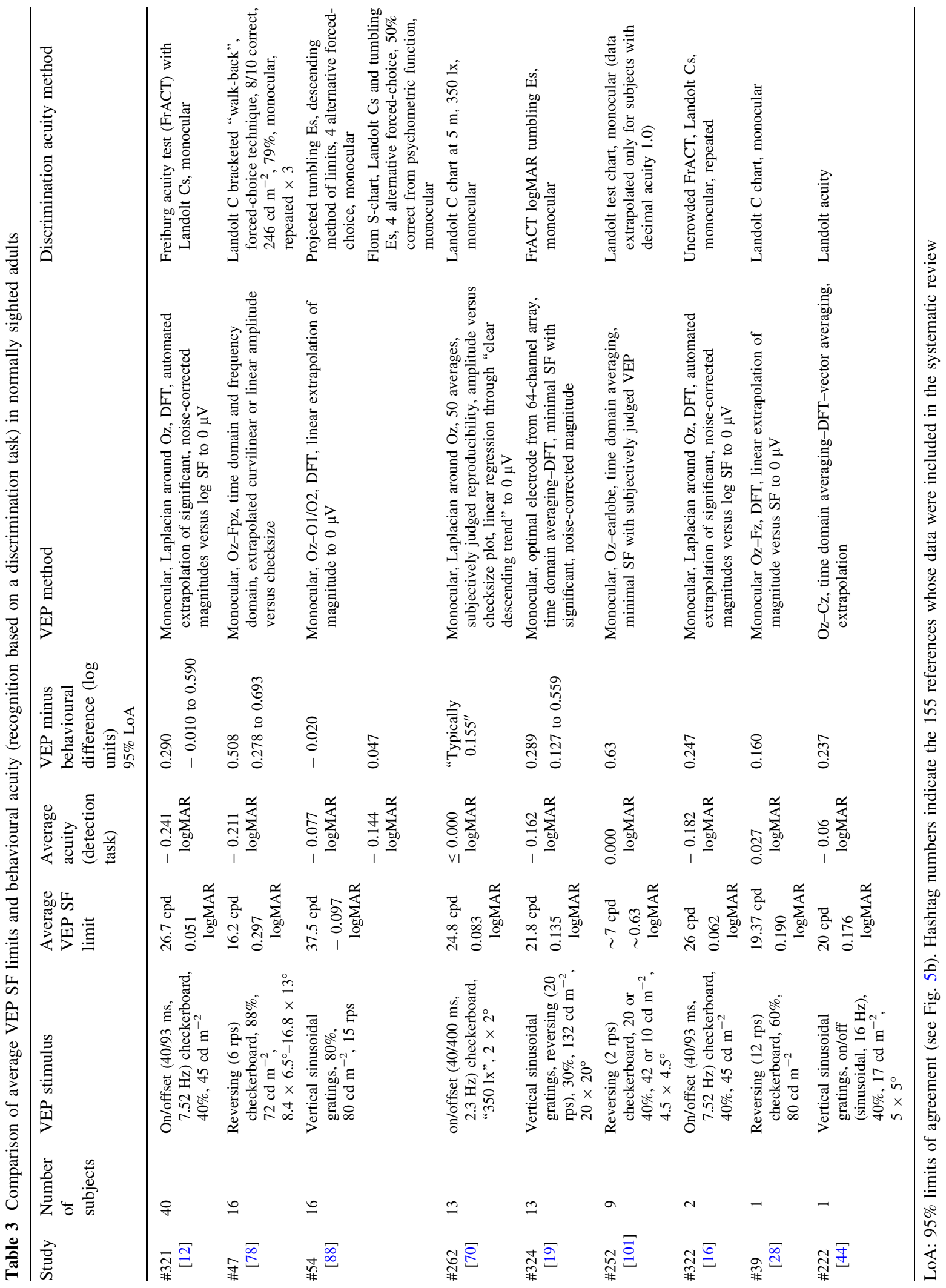


average VEP SF limits around $7 \mathrm{cpd}$ (checkwidth subtending about $6^{\prime}$ ), a $0.63 \log$ unit difference: use of transient VEPs and few pattern sizes near threshold may have resulted in subject fatigue or a floor effect causing this relatively large difference [101]. A study of 16 adults with a very thorough psychophysical acuity method also found a large VEP-psychophysical difference of $0.529 \mathrm{log}$ units based on time domain analysis of steady-state VEPs, and a slightly smaller difference of $0.487 \log$ units based on frequency domain analysis of the same data [78]. Two larger studies with 40 and 13 subjects, respectively, had very similar differences between VEP SF limits and acuity, of 0.290 and $0.289 \log$ units despite methodological differences $[12,19]$. Studies describing only one or two normally sighted adult subjects found similar VEP SF limits (19.37, 20 and 25 cpd, i.e. 0.190, 0.176 and $0.082 \log$ MAR) for average acuities of $0.027,-0.06$ and $-0.182 \log$ MAR, respectively, and hence VEPbehavioural differences of $0.163,0.236$ and $0.264 \mathrm{log}$ units, respectively $[16,28,44]$. The difference was "typically" $0.155 \log$ units in a study which noted acuities of "at least" $0.000 \log$ MAR [70]. Eight of the nine studies found better discrimination acuity than VEP SF limits, with the difference ranging from around 0.15 to $0.6 \log$ units (Fig. 5b). One study found very similar acuity and VEP SF limits using a VEP technique which also produced VEP SF limits closely matched to psychophysical thresholds for the VEP stimulus [88].

Three studies presented limits of agreement between behavioural acuity and VEP limit, or these were calculable from tabulated data: limits were wide: $\pm 0.2-0.3 \log$ units $[12,19,78]$. Together, these data suggest that, while VEP SF limits can be close to recognition (discrimination) acuity, the difference is very variable and depends strongly on the combination of VEP technique (stimulus and analysis) and acuity technique used.

\section{Non-identical stimuli-recognition (identification) acuity tests}

We identified eleven studies where normally sighted adult subjects had both VEP SF thresholds measured and also a behavioural acuity test based on an identification task, i.e. one of the many letter charts (Table 4, Fig. 5c). Again, studies were only included if sufficient detail was available to describe both average VEP SF limits and behavioural acuity: when possible, limits of agreement were also extracted. Acuities were converted from native units into $\log$ MAR units as necessary, and VEP SF limits were expressed in $\log$ MAR units to allow comparison with acuities.

Eight of the 11 studies found average recognition (identification) acuity to be better than VEP SF limits. The offset ranged from around 0.03 to $0.3 \log$ units, a smaller difference than for VEP SF limits versus discrimination acuity (Fig. 5b) and similar to the differences found using psychophysical testing with stimuli identical to VEP stimuli. The largest difference, $0.317 \log$ units, may be due to the relatively small number $(N=6)$ of SFs used [30]. Four of these eight studies found relatively small offsets of $0.1-0.2$ $\operatorname{logMAR}$ using relative high-luminance, high-contrast and large field VEP stimuli and both $\log$ MAR and Snellen standard clinical letter charts [18, 35, 55, 120], and three studies found offsets of $<0.1 \mathrm{log}$ units despite widely varying techniques [53, 84, 121]. Finally, three studies found better VEP SF thresholds than behavioural acuities. Two used high-contrast gratings, multiple SFs and objective frequency domain analysis [36, 88], while the other used low-contrast checkerboards and subjective time domain analysis with a finest SF of only $4.2 \mathrm{cpd}$, but extrapolated versus logarithmically scaled SF [118].

Several studies tabulated data, allowing $95 \%$ limits of agreement to be calculated: these were around \pm 0.05-0.25 log units, somewhat narrower limits than found for VEP versus discrimination acuity tasks.

These findings for normally sighted adults indicate that it is usual for psychophysical or clinical acuity measures to be better than VEP SF limits. For this reason, inferring a behavioural acuity of 0.000 $\log$ MAR because of a VEP SF limit of $30 \mathrm{cpd}$ in adults is very likely to underestimate behavioural acuity: in general, attributing a behavioural acuity which is the geometric equivalent of the VEP SF limit to an individual will be incorrect. An empirical calibration factor (additive offset on a log scale) is required before inferring behavioural acuities from a VEP SF limit: the value of this offset is highly variable and strongly dependent on both the VEP stimulus and analysis process and on the behavioural test (Fig. 5). The value of the offset for adults has been derived in detail in some instances, e.g. $17.6 \mathrm{deg}^{-1}(0.232$ $\log$ MAR) over a wide range of acuity [12], but is 


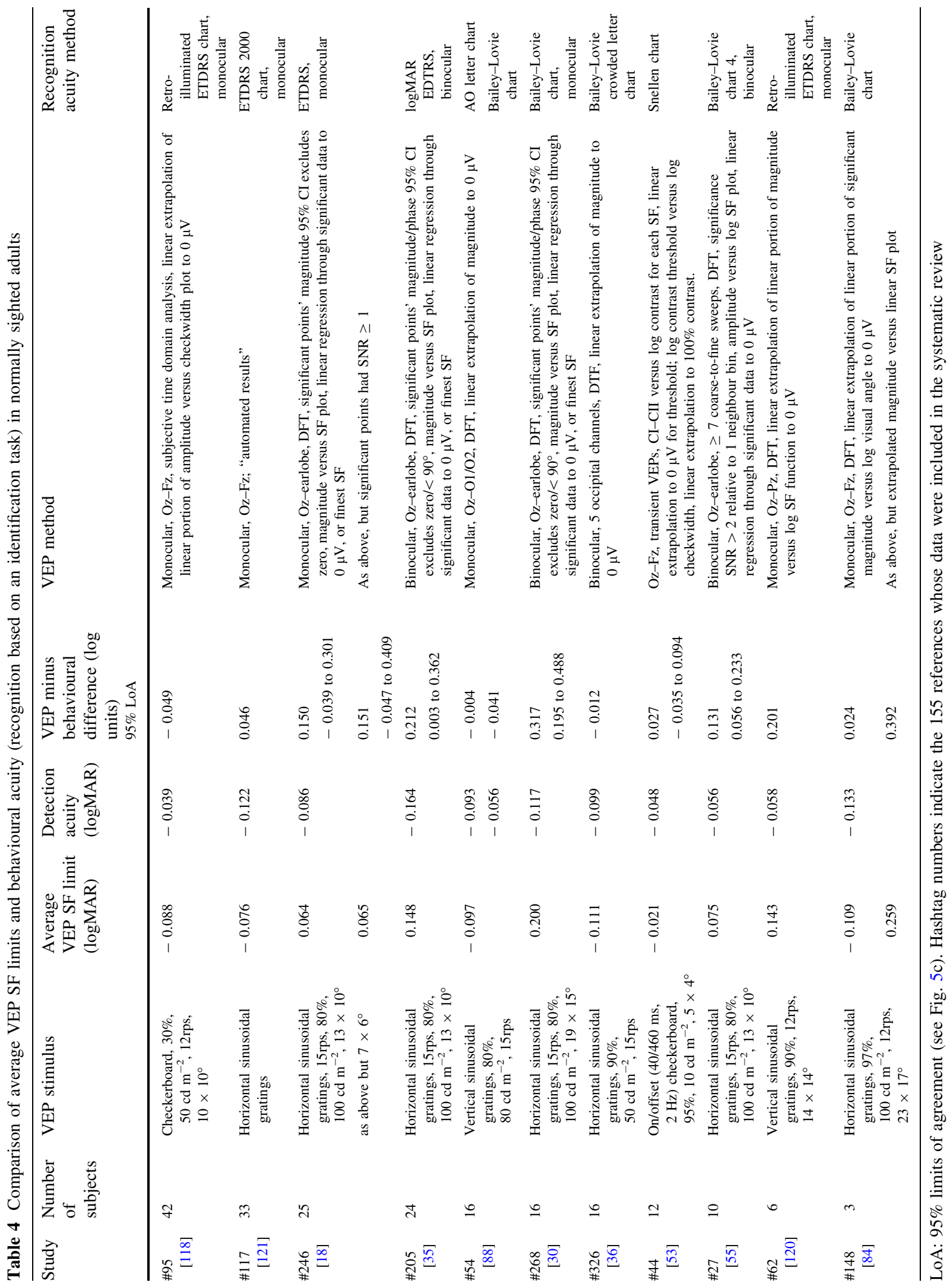


unlikely to apply to different combinations of VEP and behavioural acuity test protocols. Theoretical reasons for the offset, outlined in the introduction, are based on the different requirements of a VEP task and a psychophysical acuity test, including differences in neural substrates, inherent SNR, thresholding technique and stimulus properties.

A small VEP-behavioural offset is not necessarily a desirable goal: any offset can be handled by a calibration factor or allowed for in clinical interpretation of the VEP SF limit. A likely greater obstacle for clinical use of VEP SF limits as a proxy for acuity is the width of the limits of agreement generally found for VEP SF limits and behavioural acuity (Fig. 5b, c).

VEP SF limits in typically developing infants and children

VEP estimation of acuity is a critical tool in paediatric vision testing, particularly where co-morbidities such as cerebral palsy (CP), cerebral visual impairment (CVI) or eye movement disorders reduce the usefulness of conventional clinical acuity tests such as matching recognition tests or acuity card tests based on fixation preference. Paediatric clinics also require tools to assess children suspected of having nonorganic vision loss (NOVL) and VEP estimation of acuity can be useful in this role. The utility of VEP SF limits for all clinical applications is heavily dependent on a detailed understanding of what is "normal", i.e. the range of VEP SF limits in typically developing infants and children.

Effect of stimulus, acquisition and analysis variables on VEP SF limits of typically developing infants and children

Compared with adult studies, relative few workers have explored the effects of stimulus, acquisition and threshold estimation techniques in children. In general, VEP techniques optimised for adults have been modified, e.g. by altering the SF range used, to be useful for testing infants and children.

Some studies have investigated the temporal effects of stimuli, which is relevant due to the potentially confounding effect of maturation of temporal tuning on maturation of spatial tuning, i.e. acuity development [39]. However, no reversal rate effect on VEP SF limits was found for 10, 14 and 24 rps recorded from
42 infants ranging from 2 to 13 months old [122], nor for 12 and 20 rps in 4 and 6 month infants [123], nor for 6 and $8 \mathrm{rps}$ in 10-39-week-old infants [23]. Similarly, changing reversal rates from 12 to 15 to 20 rps did not affect VEP SF limits in 6-8-year-old children [37]. Orel-Bixler and Norcia compared VEP SF limits over the first 6 months of life for two different stimuli: transient, brief on/offset patterns of five SFs and steady-state reversing patterns of 19 SFs: for the youngest subjects, VEP SF limits were better with the steady-state stimuli; thereafter, VEP SF limits converged, matching by around 3-4 months of age, and agreeing quite closely up until 6 months of age [124]. In a study designed to investigate whether the infant retina generates high SF distortion products which evoke VEPs, VEP SF limits were slightly better with brief on/offset $5.5 \mathrm{~Hz}$ stimuli than reversal (11 rps) stimuli ( 8.8 vs. $6.7 \mathrm{cpd}$ ) in 18 infants aged 6-17 weeks [125].

VEP SF limits in 15-20-week-old infants improved nonlinearly as stimulus mean luminance increased from $0.01(\approx 2.5 \mathrm{cpd})$ to $100 \mathrm{~cd} \mathrm{~m}^{-2}$ ( $\approx 7 \mathrm{cpd}$ ); most improvement occurred between 0.01 and $1 \mathrm{~cd} \mathrm{~m}^{-2}$ [14]. VEP SF limits did not differ in 6-8year-old children across mean stimulus luminances of 25, 50 and $100 \mathrm{~cd} \mathrm{~m}^{-2}$ [22]. Similarly, typically developing children aged 3-12 years had similar VEP SF limits to low luminance stimuli (14-35 cpd, $\left.20 \mathrm{~cd} \mathrm{~m}^{-2}\right)$ as those to high-luminance stimuli (13-31 cpd, $109 \mathrm{~cd} \mathrm{~m}^{-2}$ ) [126].

It was noted that children were more attentive to coarse-to-fine SF changes than fine-to-coarse, but there were no significant differences in VEP SF limit with direction of SF change [88]. Almoqbel et al. found better VEP SF limits with coarse-to-fine than fine-to-coarse SF changes (38 vs. $31 \mathrm{cpd}$ ) in a small group of 6-8-year-old children: this difference disappeared when a fixation mark was used [37]. The same study found neither field size $\left(2,4\right.$ or $\left.6^{\circ}\right)$ nor stepwise sweep duration $(10,15$ or $20 \mathrm{~s})$ to have a significant effect on VEP SF limits. No other studies were found which investigated stimulus effects in typically developing infants and children.

A large developmental study over the first year of life showed binocular VEP SF limits to be slightly better than monocular limits at all ages by $<0.06 \mathrm{log}$ units ( $<0.2$ octaves), with a trend for the difference to lessen with age. The binocular and monocular maturation curves were very similar, and the binocular- 
monocular difference was markedly smaller than quoted for behavioural acuity (0.18-0.3 log units, i.e. 0.6-1 octaves) [127]. Similarly, for a small group of children under 5 years of age, average VEP SF limit was $24.3 \mathrm{cpd}$ with binocular viewing, and only slightly poorer $(22.9 \mathrm{cpd}$ : a difference of $0.03 \mathrm{log}$ units) with monocular viewing [88].

Infant and child studies have employed one channel or more channels in approximately equal proportion. For two or more channels, data from whichever channel or channel combination gives the "best" results tends to be used. Most commonly, an active electrode at $\mathrm{Oz}$ and/or electrodes symmetrically and laterally positioned close to $\mathrm{Oz}$, for example at $\mathrm{O} 1$ and $\mathrm{O} 2$, or PO7 and PO8, are used. Reference sites are either distant, e.g. Cz, Fpz or earlobe, or near, e.g. O1 and $\mathrm{O} 2$ active sites referenced to $\mathrm{Oz}$. A one-dimensional Laplacian montage was shown to detect ssVEPs more often and a few seconds faster than an $\mathrm{Oz}-\mathrm{Fz}$ electrode montage in children older than about 5 years, although VEP SF limits were not recorded [94].

\section{Effect of age on VEP SF limit}

As for behavioural acuity, VEP SF limits show marked maturation effects. VEP SF limits are popular as a biomarker for brain development in studies of infant nutrition, thus there is a large body of data describing VEP SF limits in typically developing infants across all nutrition groups. Additionally, many diverse studies of pathology include data from control groups of typically developing children.

We identified 52 studies which described VEP SF limits from infants and/or children screened or understood to be typically developing, and born at full-term. For nutritional studies, sub-groups were combined where possible to reflect the typical population. Extracted data were expressed in units of cpd (Fig. 6). The few data available in the first month of life suggest a rapid improvement in VEP SF limit from poorer than $1 \mathrm{cpd}$ in the first few days [128] up to $1.5-9 \mathrm{cpd}$ by the end of the first month $[32,33,124,129,130]$. As evident in Fig. 6, there is rapid improvement until 8-12 months when VEP SF limits are typically $15-20 \mathrm{cpd}[14,15,17,20,21,23$, 24, 26, 27, 32, 33, 38, 71, 73, 122-124, 127, 129-154]. A control group of 27 infants aged 6-25 mo had a mean VEP SF limit of $13.4 \mathrm{cpd}$ with a trend towards better limits with age [155].

From 1 year through to adulthood a slower improvement is evident, from 12 to $20 \mathrm{cpd}$ up to 20-40 cpd [17, 22, 26, 27, 32, 33, 37, 38, 72, 73, 88, $132,156,157]$.

In some cases, these values or trends were not observed. Riddell et al. tested 35 infants aged from 1 to 10 months, and found good VEP SF limits from the youngest infants $(<4$ mo, 4-11 cpd) with little improvement for the oldest infants $(8-10$ months, 8-11 cpd), giving a flatter developmental curve [129]. Data from Sokol et al. followed an underlying trend of improvement for ages 2-5 mo, but at 6 and at 7 months, infants had excellent and adult-like VEP SF limits (29 and $42 \mathrm{cpd}$, respectively), although only nine and two subjects, respectively, contributed to these averages [139]. Similarly, three subjects aged 5-6 months had adult-like VEP SF limits of around $30 \mathrm{cpd}$ [124] and eight subjects aged 3-7 months had adult-like limits of 20 or $30 \mathrm{cpd}$ [130]. In a crosssectional study of 61 infants aged 6-9 months, there was a large spread of VEP SF limits (3-14 cpd) but no age-related trend [150].

Poorer VEP SF limits than those described above, by around $10 \mathrm{cpd}$, were reported for 40 control children aged 1-13 years, perhaps partly due to a long study protocol which included VEP contrast limits, behavioural acuity assessment, retinoscopy and accommodation assessment [158]. A large group of 55 children aged 5 years had average VEP SF limits of around $12 \mathrm{cpd}$ [134], at the lower limit of typical limits (15 cpd or better) reported for similar ages [37, 72, 73, 79, 88, 132]: again, children undertook a lengthy protocol including anthropometric, neurodevelopment and multiple vision assessments.

Despite these examples, the majority of studies illustrate a trend of rapid improvement from 1.5 to $9 \mathrm{cpd}$ at 1 month to $12-20 \mathrm{cpd}$ by $8-12$ months, followed by slower improvement to $20-40 \mathrm{cpd}$ by adulthood. This consistency may be partly because a majority of paediatric studies use versions of the same stepwise sweep VEP technique developed by Tyler, Norcia and colleagues [29, 159]. However, VEP SF limits established using quite different techniques, for example on/offset transient VEPs, show a very similar developmental curve [38].

As with all forms of paediatric testing, success rates for establishing a VEP SF limit vary with age. Success 

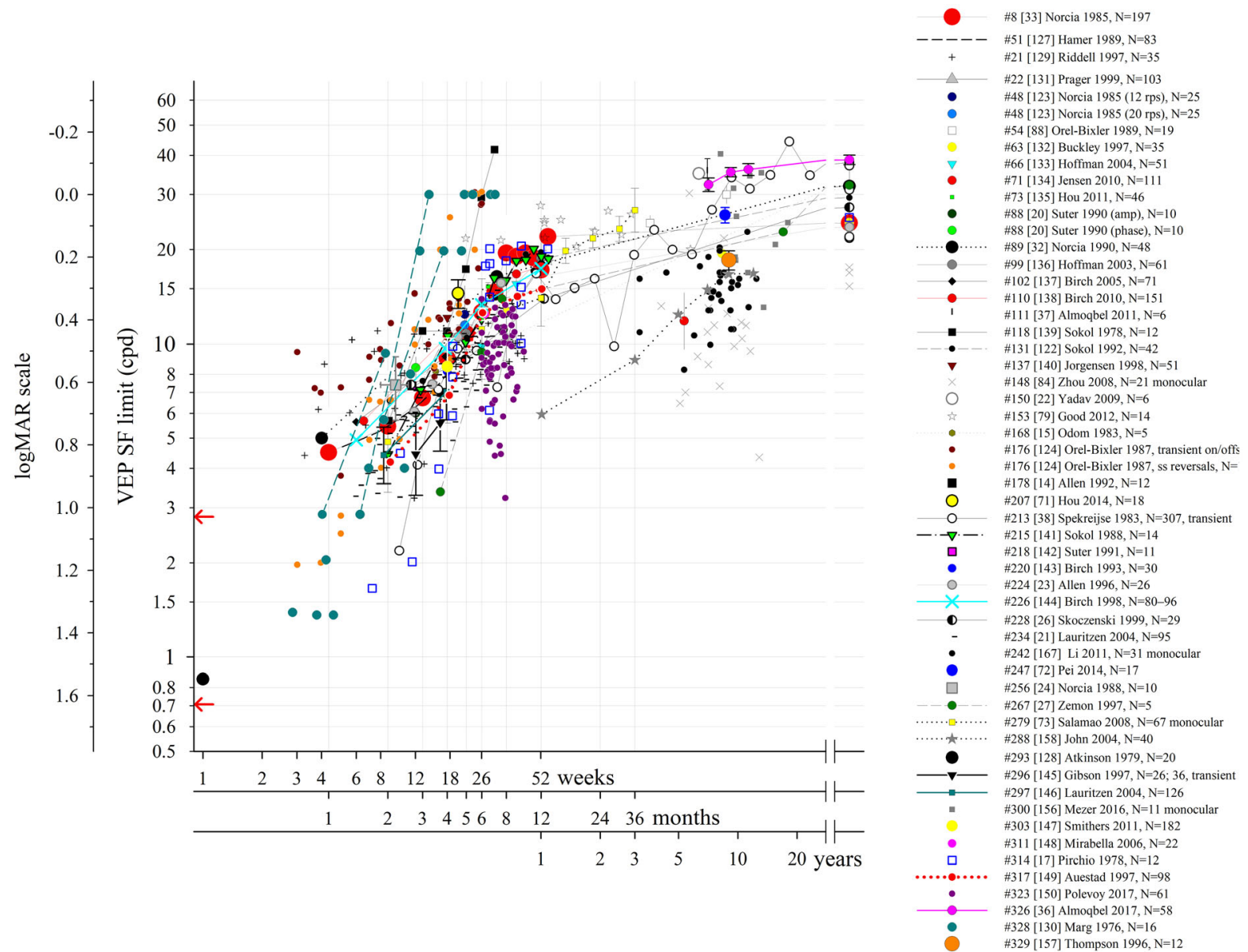

Fig. 6 Illustration of the development of VEP SF limits through infancy and childhood from 52 studies. A variety of techniques were employed (see text for details). Dashed lines indicate subjects from a cross-sectional study. Solid lines

could be defined as collection of sufficient and adequate data to define a VEP SF limit. Some studies of typically developing infants and children report high success rates, such as 83/87 (95\%) [127] and 197/215 (92\%) [33] in infants around the first year of life: failures occur in the youngest and oldest subjects. Similarly, data were recorded from 142/147 (97\%) 2-month-old infants, and VEP SF limits defined for 126 of the 147 (89\%): this improved to $147 / 148$ (99\%) and $147 / 148(99 \%)$ for the same subjects at 4 months [146]. This improved success may have been due to increased infant maturity, interest and/or cooperation, but also to increased parent familiarity with the procedure, or improved skill of the researchers [146]. Other studies report lower success rates, such indicate participants in a longitudinal study. Error bars typically indicate SEM. Red arrows at the SF axis indicate the two ISCEV standard checkwidths, $60^{\prime}$ and $15^{\prime}(0.71$ and $2.8 \mathrm{cpd})$

as data recorded from 48/52 (93\%) 12-week-old infants but VEP SF limits defined for only $26 / 52$ (50\%): again, this improved to 52/52 and 36/52 (69\%) for the same subjects at 16 weeks [145]. All 44 older children (3 months to 14 years) had data successfully collected, but VEP SF limits were defined for only 40/44 (91\%) [158]. Children can be particularly erratic in compliance around 2-4 years, and VEP SF limits drop markedly in this age range in a large crosssectional study, suggesting that compliance not only affects success in obtaining a VEP SF limit, but perhaps also the limit itself [38]. 


\section{Effect of premature birth on VEP SF limit}

The effect of premature birth has been investigated by assessing prematurely born infants without sequelae such as retinopathy of prematurity or cerebral injuries, or only mild forms of these, often termed "healthy" preterm infants. One study found slightly but significantly better VEP SF limits (0.14 log units) in 13 healthy infants born prematurely (31.4 ( \pm 3.3 SD) weeks gestation) and tested at post-term ages of 1-10 months, relative to term-born, age-matched controls. Comparison by post-natal age showed no significant difference, suggesting VEP SF limits develop from birth, and therefore that premature birth might accelerate development [160]. No other study with term-born, age-matched controls found this accelerated development; however, 17 infants born prematurely (at 27-32 weeks gestation) and tested at 4 weeks preterm (36 weeks post conception) and at 17 weeks post-term age had VEP SF limits of 4.4 and $13.7 \mathrm{cpd}$, respectively [161], which are towards the upper end of values reported for term-born infants (Fig. 6). In contrast, four studies each with a termborn, age-matched control group found similar or slightly poorer VEP SF limits in healthy prematurely born infants. Prematurely born versus term-born infant VEP SF limits were 12.4 versus $12.5 \mathrm{cpd}$ [148] and 12.7 versus $15.2 \mathrm{cpd}$ at 6 months corrected age [135]. Two nutritional studies found very similar VEP SF limits (around $8 \mathrm{cpd}$ ) in healthy, prematurely born and in term-born control infants at 4 months corrected age [143, 162].

Several other nutritional studies recorded VEP SF limits in healthy, prematurely born infants: these study designs did not include a control group of term-born infants, but limits fitted well within the values collated from typically developing, term-born infants (Fig. 6). Average VEP SF limits for healthy prematurely born infants have been reported as $5.6 \mathrm{cpd}$ at 2 months post-term [163]; 7.4, 8.5 and 8.9 cpd at 4 months postterm ages $[83,163,164]$ and $8.9,11.5$ and $13.1 \mathrm{cpd}$ at 6 months post-term [83, 164, 165]. For a large crosssectional cohort of low- and high-risk prematurely born infants assessed using transient VEPs, VEP SF limits improved from around $2 \mathrm{cpd}$ at term age to around $3.3 \mathrm{cpd}$ at 10 weeks post-term and around $6 \mathrm{cpd}$ at 2 years post-term [166], suggesting a flatter developmental curve than seen in typically developing term-born infants (Fig. 6).
Collectively, these findings suggest that prematurely born infants who largely escape ophthalmic or neurological complications of prematurity are likely to have similar VEP SF limits to their term-born peers, making any adjustment for prematurity unnecessary for healthy preterm infants.

Correspondence of VEP SF limits

with behavioural thresholds in typically

developing infants and children

\section{Identical stimuli}

Two groups have compared VEP SF limits with psychophysically measured thresholds to identical stimuli in infancy and childhood. Sokol et al. undertook a mixed cross-sectional and longitudinal study in 14 typically developing infants between 2 and 13 months. VEP SF limits improved from 4 to $19 \mathrm{cpd}$, while preferential-looking thresholds improved from 1 to $14 \mathrm{cpd}$ over the same age range (Fig. 7, left). Some infants were also tested with a stationary version of the preferential-looking task, i.e. the stimulus did not reverse, which gave similar but slightly poorer thresholds [141]. The same group compared VEP SF limits and preferential-looking thresholds in 42 typically developing infants aged 2-13 months with very similar results, showing VEP SF limits much better than behavioural thresholds at the youngest ages tested (around $0.6 \log$ units better at 2 months): this difference diminishes with age, approaching zero difference by the end of the first year of life [122] and closely matching the near-zero difference observed in adults [141]. Using a temporal, 2-alternative forced-choice staircase procedure, psychophysical thresholds were compared with VEP SF limits using identical stimuli, albeit with a smaller field size for the psychophysical measurements $\left(4 \times 4^{\circ}\right.$ vs. $\left.10 \times 10^{\circ}\right)$ in 48 older children (6-12 years old) and adults. Both measures agreed closely, with slightly better psychophysical thresholds at all ages [36].

\section{Non-identical stimuli-recognition acuity tests}

We identified 14 studies which compared behavioural acuities with VEP SF limits in typically developing infants and children (Fig. 7 right). In all studies investigating children under 1 year, Teller acuity 

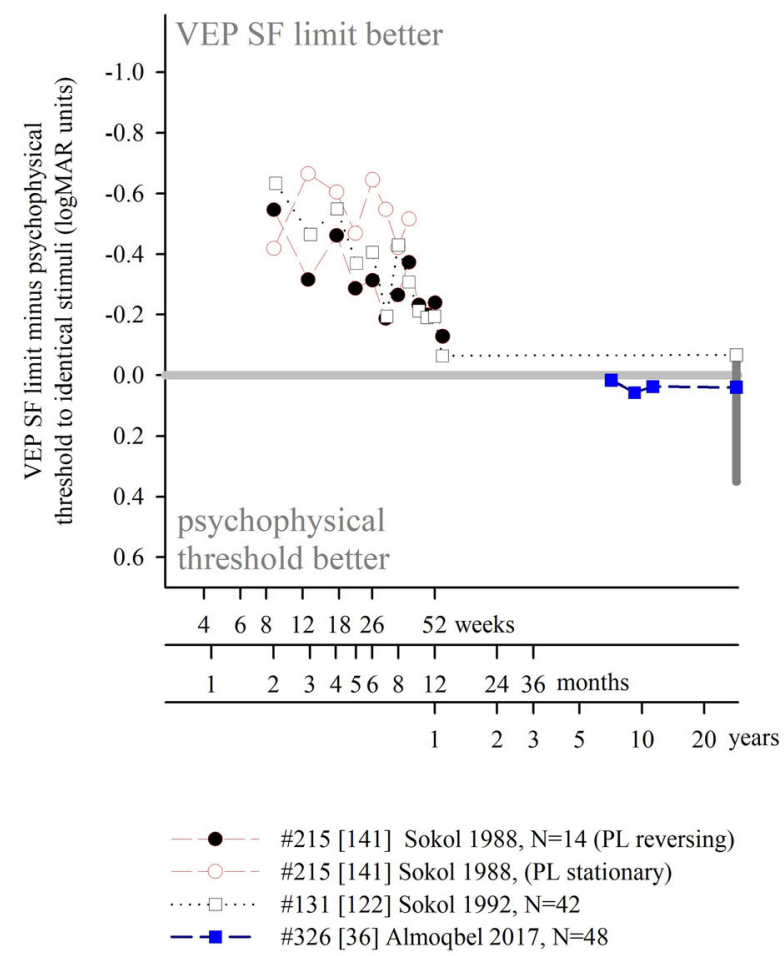

Fig. 7 Illustration of difference between VEP SF limits and acuity and its change with age. Left panel: psychophysical acuity tests using identical stimuli to VEP test. Right panel: behavioural tests using age-appropriate clinical acuity tests.

cards (TAC) or a digitally rendered similar test was used. At the youngest ages tested ( $\lesssim 10$ weeks), VEP SF limits were consistently and markedly better, by 0.2-1.0 log units, than behavioural testing [129, 144, 149]. All used some form of extrapolation to define the VEP SF limit. This difference lessened with age: by 4 months of age, several studies found VEP SF limits to be better than behavioural thresholds by only around $0.2-0.35 \quad \log$ units [131, 143, 144, 149, 162]. Towards the end of the first year of life, the difference was less still but inconsistent, with some studies finding better behavioural acuity than VEP SF limits [131, 150] and some the opposite [129, 144, 149, 162].
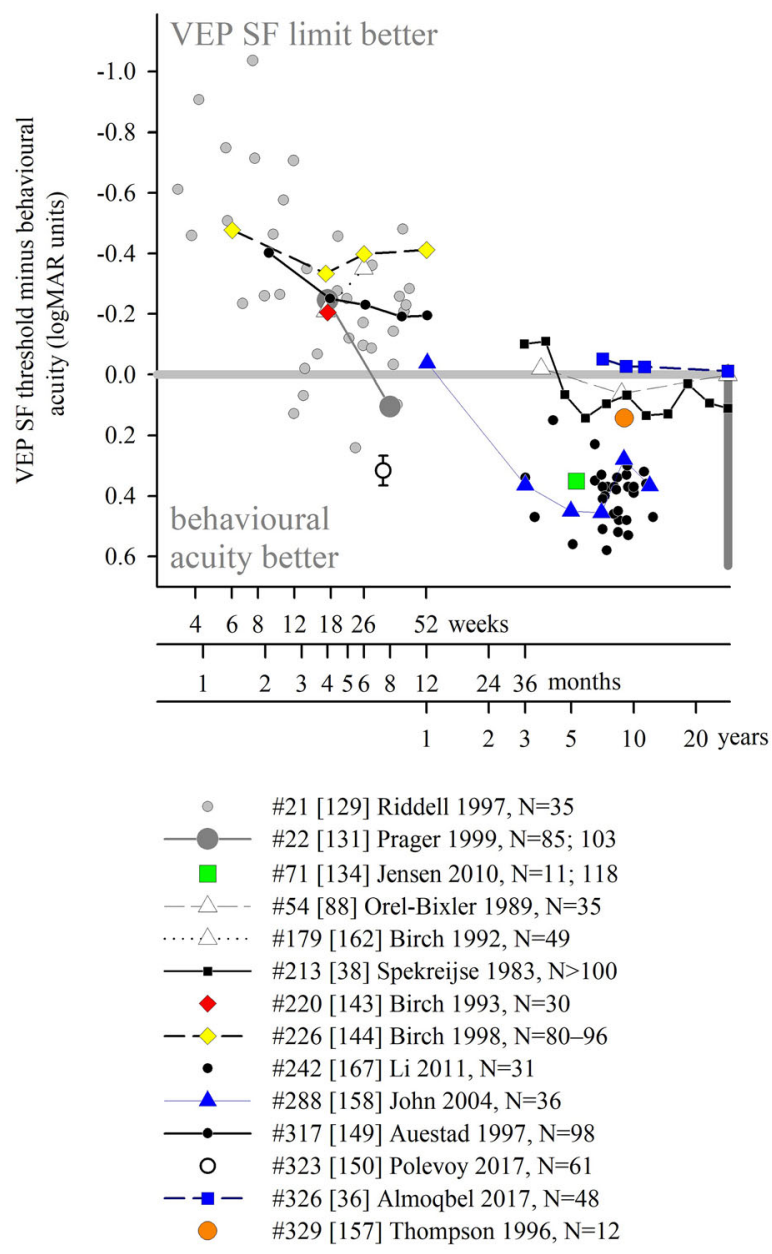

Solid grey horizontal line indicates no difference. Solid vertical line at adult ages indicates typical range of differences reported in healthy, normally sighted adults (see Fig. 5). Error bars represent SEM. PL: preferential-looking

Studies of children aged 3 up to adulthood found behavioural acuity either closely matched or up to 0.6 $\log$ units better than VEP SF limits [36, 38, 88, 134, 149, 157, 158, 167]. Three crosssectional studies using a mixture of age-appropriate acuity tests found very closely matched thresholds, with a slight tendency for behavioural acuities to be better than VEP SF limits in older children and adults $[36,38,88]$. Four other studies found a more marked difference, with behavioural acuities better by around $0.4 \log$ units [134, 149, 158, 167].

We were unable to find any extractable data comparing VEP SF limits with behavioural acuity for children aged 12-36 months, although a study of 
35 typically developing infants and children aged 1-36 months noted VEP SF limits exceeded forcedchoice preferential-looking acuity in the first year, but matched closely in the second and third years of life [157]. Only one cross-sectional study spanned these ages and found a marked change over this interval, with closely matched thresholds at 12 months, but markedly better behavioural thresholds by 36 months [158].

These data support the conclusion that VEP SF limits are much better than behavioural thresholds in the youngest, typically developing infants, but that this difference lessens with age, with no difference expected somewhere between the first and second year of life. From around 3-5 years, the same pattern is observed as is seen in healthy, normally sighted adults, i.e. behavioural acuity tends to be better than VEP SF limit. It was established earlier that normally sighted adults usually have behavioural acuity which is better than their VEP SF limits by 0 to $0.6 \log$ units. The difference shows high inter-subject and betweenstudies variability at all ages and depends on both the VEP SF limit technique and the behavioural acuity test.

Correspondence of VEP SF limits

with behavioural thresholds in normally sighted

adults with artificially degraded vision

We identified 21 studies where normally sighted adults had both behavioural acuity and VEP SF limits measured, while their vision was degraded using either Bangerter occluding foils [12, 69, 92, 101, 111, 121], plus lenses [19, 29, 30, 43, 44, 54, 100-103, 116, 120, $168,169]$, scatter transparencies [16] or frosted panes [112]. Where possible, data were extracted and converted into $\operatorname{logMAR}$ units for both behavioural acuities and for VEP SF limits. If adjustments for behavioural versus VEP offsets had been applied [12, $16,69,112]$, these were removed to allow comparison with unadjusted data from other studies (Fig. 8).

Fifteen of the 21 studies found typical VEP SF limits to be poorer than perceptual acuity by around 0.2-0.6 log units $[12,19,29,30,43,44,54,92,100$, $101,103,111,112,168,169]$. Most of these studies also found this behavioural acuity versus VEP SF limit offset to be constant over the range of acuities assessed [12, 19, 43, 44, 54, 92, 101, 111, 169], albeit with a ceiling effect evident in one study which found the same VEP result for all acuities better than 0.5 logMAR (using $80 \%$ contrast checks), and similarly for acuities better than about $0.2 \log$ MAR (for $40 \%$ contrast checks) [100]. This consistent behavioural acuity versus VEP SF limit offset was evident despite large differences in methods, i.e. VEP pattern, contrast, number of SFs, process of determining threshold, behavioural test and means of degrading vision. Four of these 15 studies found the behavioural acuity versus VEP SF limit offset changed over the range of acuities assessed, with the offset narrowing towards poorer acuities in three [30, 103, 168] but widening markedly in one study using a continuous sweep VEP technique to compare VEP SF limit with psychophysical acuity using an identical stimulus in one defocussed individual [29].

Two further studies found poorer behavioural acuities than VEP SF limits at all acuities assessed, but most marked at the poorest acuities measured [116, 120].

Four further studies found approximately equal behavioural acuities and VEP SF limits, i.e. an offset of approximately zero $[16,69,102,121]$. One of these investigated subjects with markedly degraded acuity ( $\approx 2.0 \log$ MAR) [16]; this close-to-zero offset was different from the $0.232 \log$ MAR offset found using identical techniques in a large group of subjects degraded to acuities of $\approx 1.0 \log$ MAR or better [12] and may therefore indicate a different calibration factor for the low vision range. Three further studies, all using high contrast patterns, also found little or no offset, with VEP SF limits even being slightly better than behavioural measures at the poorest acuities ( $\approx 1.0 \log$ MAR or better [69], or at around 0.3 logMAR [121]). The third used transient on/offset VEPs fitted with a parabolic or modified Ricker function in eight defocused subjects to compare with Snellen chart acuities of 1.0 logMAR and better [102].

One study directly compared plus lenses with Bangerter foils and found Bangerter foils gave slightly better (by around $0.15 \mathrm{log}$ units) VEP SF limits than plus lenses for the same behavioural acuities [101]. While not specifically identified in that study, optical defocus can cause spurious overestimation of both perceptual and VEP SF limits to periodic stimuli due to the emergence of a phase-inverted, lower contrast image as dioptric blur increases [170, 171].

One study incorporated two behavioural tests, a letter chart and psychophysical acuity using the same 


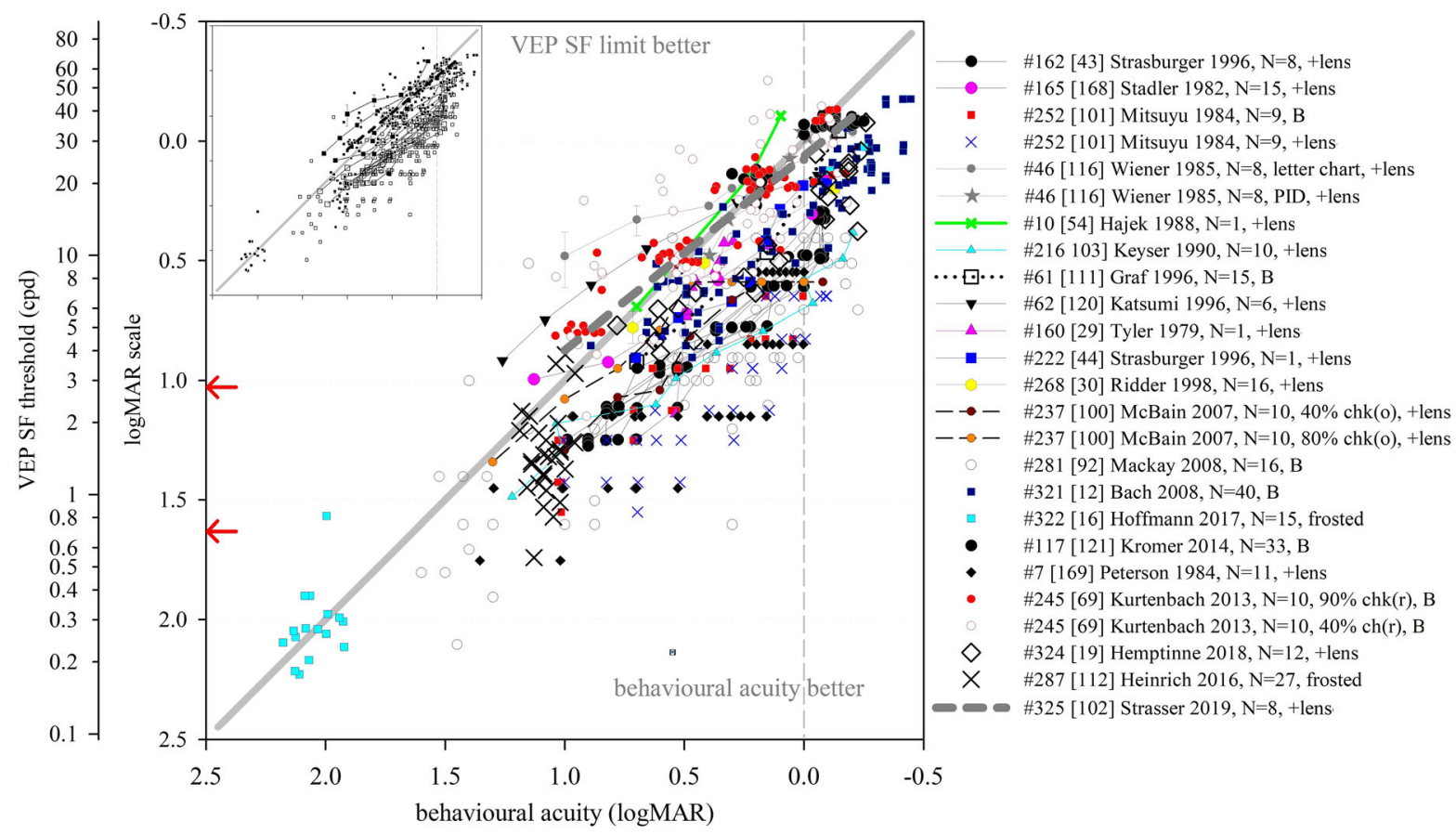

Fig. 8 VEP SF limit versus behavioural acuity in healthy, normally sighted adults with artificially reduced acuity. Legend indicates study ID, number of subjects $(N)$, and means of reducing acuity. Solid grey line: equality. Vertical dashed grey line: $0.0 \log$ MAR. PID psychophysical test with stimuli identical to that used for VEP SF limit. Lines join symbols representing individual subjects if known, or join mean data from the same group of subjects. Where adjustments for

stimulus as for the VEP. VEP SF limits closely matched psychophysically measured acuity across all acuities assessed $(\approx 0-0.4 \log$ MAR $)$, while VEP SF limits were markedly better than letter chart acuity at poor acuities, but somewhat worse at good acuities [116].

VEP SF limits in clinical conditions

This section aims to compile what is known about VEP SF limits in a range of clinical conditions affecting vision such as cataract or macular disease, or in patients with clinical signs such as nystagmus, to establish its accuracy and precision and to comment on the suitability of using VEP SF limits to estimate acuity. behavioural versus VEP offsets were used [12, 16, 69, 112], these have been removed to allow comparison with unadjusted data from other studies. Red arrows at the SF axis indicate the two ISCEV standard checkwidths, $60^{\prime}$ and $15^{\prime}(0.71$ and 2.8 cpd). Inset: the same data are presented to illustrate a closer VEP-behavioural match for those studies using extrapolation techniques (closed symbols) than those using the finest SF technique (open symbols)

\section{Heterogeneous patient groups}

Several studies of VEP SF limits in patients investigated heterogeneous patient groups in order to establish real-life utility of the technique. General findings from such studies are presented initially: subsequent sections present findings by specific conditions or signs, ordered in an anterior to posterior direction along the visual pathway, i.e. beginning with conditions which affect optical input (media opacities, refractive error, nystagmus), then retinal and macular disease, followed by diseases of the optic nerve and finally covering conditions where the primary lesion is cerebral, i.e. amblyopia and neurological conditions. NOVL (non-organic vision loss) is treated last.

We identified eight studies of VEP SF limits in heterogeneous patient groups; data were exractable from six studies (Fig. 9). VEP SF limits 


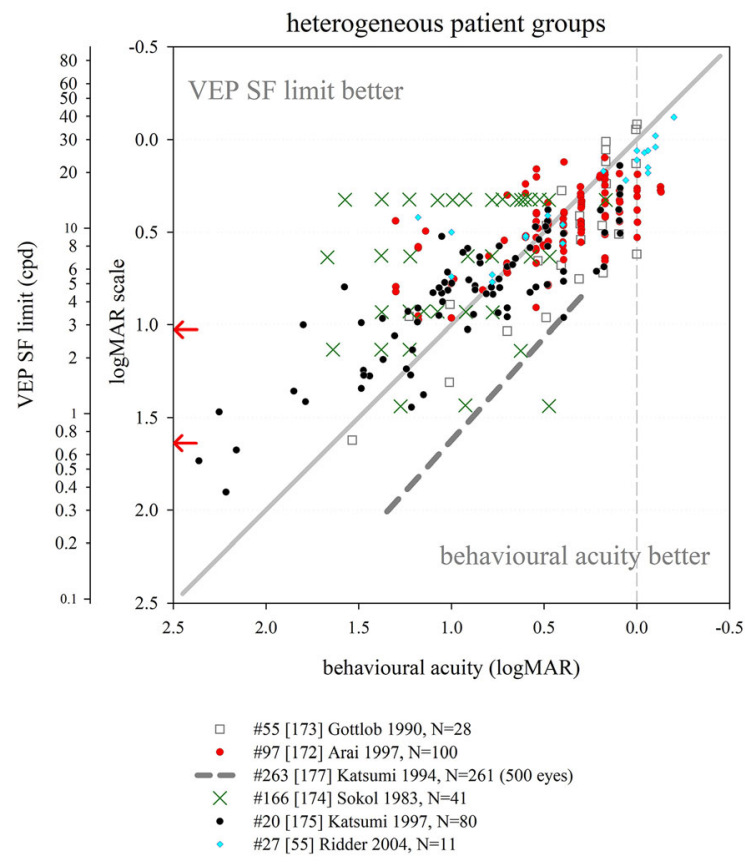

Fig. 9 VEP SF limit versus behavioural acuity in heterogeneous groups of patients. Solid grey line: equality. Dashed vertical grey line: indicative normal behavioural acuity limit (0.0 $\log \mathrm{MAR})$. Red arrows at the SF axis indicate the two ISCEV standard checkwidths, $60^{\prime}$ and $15^{\prime}(0.71$ and $2.8 \mathrm{cpd})$

were better than behavioural acuities in 329 ophthalmic patients ( $8-85$ years) with diverse conditions impairing their vision; this overestimation was more pronounced at poorer acuity levels, e.g. VEP SF limits around $0.65 \log$ MAR when behavioural acuity was around 1.5 logMAR [118]. Average VEP SF limits were similar to average behavioural acuities in 100 patients (7-90 years) with diverse conditions impairing their vision, but VEP SF limits exceeded acuity at poorer acuity levels, e.g. VEP SF limits around 0.7 $\log$ MAR when behavioural acuity was around 1.0 $\log$ MAR. The gap lessened as acuity improved; for patients with behavioural acuities better than about $0.45 \log$ MAR, VEP SF limits fell short of behavioural acuities [172]. Similarly, VEP SF limits in 11 patients (3-81 years) with poor acuity due to diverse conditions were poorer by around $0.14 \log$ MAR on average compared with letter chart acuity; however, for the poorest acuities $(\approx 1.0 \log \mathrm{MAR})$, VEP SF limits exceeded behavioural thresholds by around 0.7 logMAR: again, this gap lessened as acuity improved [55]. A sweep VEP method was highly successful
$(>95 \%)$ and strongly correlated with behavioural acuity in a group of 135 patients aged 3 weeks to 11 years with diverse visual disorders. Behavioural acuities were typically around $0.1 \log$ MAR better than VEP SF limits (Fig. 9), but the age span included ages when VEP-behavioural differences in typically developing children still reverse (cf. Fig. 7) [173]. VEP and a forced-choice preferential-looking technique had approximately equally success in children $<2$ years old for binocular testing, but for monocular testing, the VEP technique (transient; six checkwidths with $\mathrm{SF}_{\mathrm{f}}$ 5.7-240 cpd) was markedly more successful in children aged 3 years or under [174]. In the same study, for 41 patients (median age 1 year) with visual problems, binocular VEP SF limits were almost always better than behavioural acuities (average of 0.64 vs. $1.01 \log$ MAR) — again, this age span typically shows a marked reversal in VEP-behavioural differences (cf. Fig. 7) [174]. Monocular VEP SF limits recorded from 80 paediatric patients (1.5 months to 12 years) with diverse visual disorders and a broad span of acuities agreed closely with behavioural acuities measured using a stationary version of the VEP stimulus and a forced-choice, preferential-looking or pointing/verbal responses staircase procedure (Fig. 9). As in the studies above, VEP-behavioural difference varied with acuity: for patients with the poorest behavioural acuities $(\approx 2.25 \log$ MAR $)$, VEP SF limits were better $(\approx 1.6 \log \mathrm{MAR})$, while the opposite was found for patients with good acuity ( $\approx 0.1 \log$ MAR), whose VEP SF limits were poorer at around $0.4 \log$ MAR. Over $95 \%$ of patients had VEP and behavioural measures within $\pm 0.3 \log$ MAR units [175]. Since the study group ages spanned those when VEP-behavioural differences typically reverse markedly (cf. Fig. 7), it is likely that some of the change in VEP-behavioural difference with acuity is due to the normal VEP-behavioural difference changing with age observed in healthy children [175].

The studies described above follow a pattern of VEP SF limits overestimating acuity at poor acuity but matching more closely as acuity improves. Two studies deviated from this pattern. One study of 42 children (4-116 months) with visual impairment due to multiple, diverse causes reported mean behavioural acuity better than mean VEP SF limit ( 0.89 vs. 1.16 logMAR) [176]; ages spanned those when VEPbehavioural differences typically reverse markedly (Fig. 7). Also, behavioural (forced-choice 
preferential-looking) tests were more successful than VEP SF limits extrapolated from transient (3.8 rps) VEPs (41/42 vs. 27/42), with particularly poor VEP success in those with nystagmus or seizure disorders. In the largest available study of patients, VEP SF limits were consistently poorer than behavioural acuity by about $0.6 \log$ MAR units across all acuities assessed from 500 eyes of 261 patients (8-88 years): however, unlike other clinical studies, the threshold criterion was the finest SF to produce a reliable VEP, with the finest available checkwidth as large as $10^{\prime}$ (4.2 cpd, 0.85 logMAR) [177] (Fig. 9).

Despite an identical stimulation protocol and very similar patient groups, the two largest studies had markedly different findings: extrapolation to a $0 \mu \mathrm{V}$ threshold [118] using a logarithmically scaled SF axis produced substantially better VEP SF limits than the finest SF technique [177].

In summary, data from adult and older paediatric patients show that VEP SF limits exceed behavioural acuity in patients with poor acuity. In some studies, this gap lessens as acuity improves, with the two measures matching at around 0.3-0.5 $\log$ MAR [55, 172]: for acuities better than this, VEP SF limits may underestimate behavioural acuity. Comparing Figs. 8 and 9 suggests that pathologies impairing visual acuity do not cause quite the same VEP SF limit-behavioural acuity relationship as that found for artificially blurred, healthy adults. General findings in younger paediatric patients are complicated by two factors: firstly, unlike adult patients, it cannot be assumed that the behavioural acuity measure is the gold standard. Secondly, the VEP-behavioural difference is known to alter markedly over the first 3 years of life in typically developing infants and children (cf Fig. 7). Studies presenting VEP-behavioural differences in ages which include both under- and over-3year olds may therefore confound the impact of pathology with expected physiological development.

\section{Opacities}

We identified six studies where VEP SF limits and behavioural acuities were measured in patients with media opacities such as cataract or vitreous opacities, five with extractable data (Fig. 10). A large study of 59 patients with varying degrees of cataract $(N=56)$ or vitreous haemorrhage $(N=3)$ using a finest $\mathrm{SF}$ criterion found average VEP SF limits only around

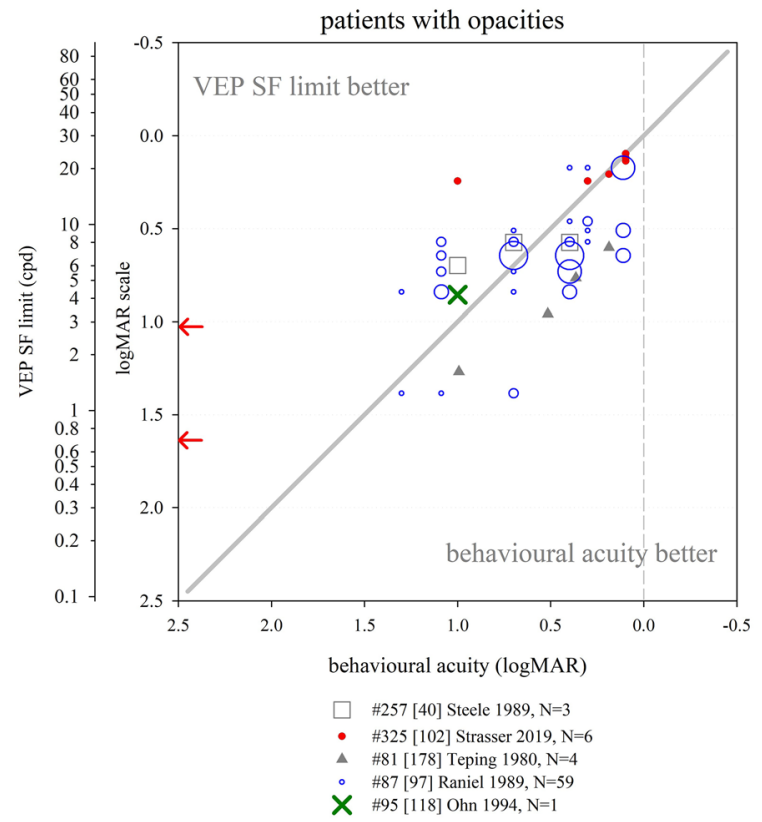

Fig. 10 VEP SF limit versus behavioural acuity in patients with opacities. Open blue circle size reflects the number of subjects with any particular pair of results. Solid grey line: equality. Dashed vertical grey line: indicative normal behavioural acuity limit (0.0 logMAR). Red arrows at the SF axis indicate the two ISCEV standard checkwidths, $60^{\prime}$ and $15^{\prime}$ (0.71 and $2.8 \mathrm{cpd})$

$0.1 \log$ unit poorer than behavioural acuities, but relatively wide $90 \%$ limits of agreement of around \pm $0.5 \operatorname{logMAR}$ [97]. In a group of 13 patients with cataracts, the average VEP SF limit was $0.26 \log$ units better than behavioural acuities: one example patient (Fig. 10) had a VEP SF limit $0.144 \log$ units better than their behavioural acuity of $1.000 \log$ MAR [118]. Similarly, two smaller patient groups $(N=6 ; 5$ cataract, 1 vitreous opacity [102]; $N=3 ; 2$ cataract, 1 vitreous haemorrhages [40]) had generally close agreement between VEP SF limit and behavioural acuity. One study found VEP SF limits in four patients with cataracts consistently poorer than behavioural acuities by around $0.4 \mathrm{log}$ units using a relatively dim $\left(10 \mathrm{~cd} \mathrm{~m}^{-2}\right)$, low-contrast (20\%) stimulus [178]. A case series of six aphakic infants had transient VEP SF limits which correlated with single letter visual acuity [179].

Collectively, these data suggest that media opacities impair VEP SF limits and behavioural acuities similarly, and the trends seen in this small group of five studies are similar to those seen in healthy, 
normally sighted adults with artificially reduced acuity using plus lenses or Bangerter foils (Fig. 8). This supports the assertion that pathologies affecting the anterior part of the eye degrade the VEP stimulus akin to blurring [169].

\section{Nystagmus}

Pattern-reversal VEPs are degraded in patients with nystagmus, whereas pattern-onset VEPs are less affected [180, 181], probably because motion adaptation caused by nystagmus-induced retinal slip is lower for pattern onset than for pattern-reversal stimulation which has a higher duty-cycle of pattern-presence and hence retinal image motion [182]. It is therefore likely that VEP SF limits will be affected by the presence of nystagmus, and that the effect size will depend on the choice of on/offset or reversal, as well as orientation of gratings. However, we did not identify any studies which described changes in VEP SF limits with choice of on/offset or reversal, or with orientation of gratings, in patients with nystagmus. Twenty-six children with mild or moderate nystagmus in association with other diagnoses, part of a cohort of 175 children, had worse VEP "scores" (deficit in log units between an individual's VEP SF limit and limits from ageexpected norms; pattern reversal) by $0.77 \log$ units than children without nystagmus (0.43 log units); similar deficits were found with behavioural testing (Teller Acuity Cards) with deficits of 0.86 and 0.52 , respectively [183]. A large $(N=172)$ group of young (median age 1 year) patients with heterogeneous causes for vision loss had better VEP SF limits (pattern reversal) on average than behavioural acuity (0.33 vs. $1.01 \log$ MAR, Fig. 9): in five patients with nystagmus, this was reversed and the VEP SF limits were about $0.15 \log$ MAR units poorer than behavioural acuity [174]. Using horizontal gratings rather than checkerboards may improve success rates with VEP SF limits: having established VEP SF limits (checkerboard reversal) in only 28/42 (67\%) paediatric patients with a broad range of aetiologies (22/42 including nystagmus) [176], the authors reported improved success in a subsequent study of 38 similar patients [184]. A sub-group (17/38) had nystagmus and so were tested with horizontal bar gratings (reversing) rather than reversing checkerboards, and VEP SF limits were established in 14/17 (82\%) [184]. Mean VEP SF limits from fellow eyes were similar when six children exhibiting latent nystagmus were removed from the original group of 12, suggesting the choice of horizontal, reversing sinusoidal gratings were robust to nystagmoid blur [157].

Despite the well-established fact of more robust transient VEPs to on/offset than reversal stimuli, and the adoption of horizontally rather than vertically oriented gratings in clinical practice [185] for patients with horizontal nystagmus [186], there appears to be surprisingly little evidence of the effects of these stimuli changes on VEP SF limits in those with nystagmus.

\section{Refractive error}

This section aims to identify the effect of uncorrected refractive errors on the VEP SF limit, and its relationship with behavioural acuity which is reasonably expected to be similar to effects of artificial blur in healthy adults (see above). Four studies were identified which measured VEP SF limits in uncorrected myopes; data were extractable from three of these. VEP SF limits recorded from 19 of 34 uncorrected myopic adults correlated well with behavioural acuities (Fig. 11): the 15 patients from

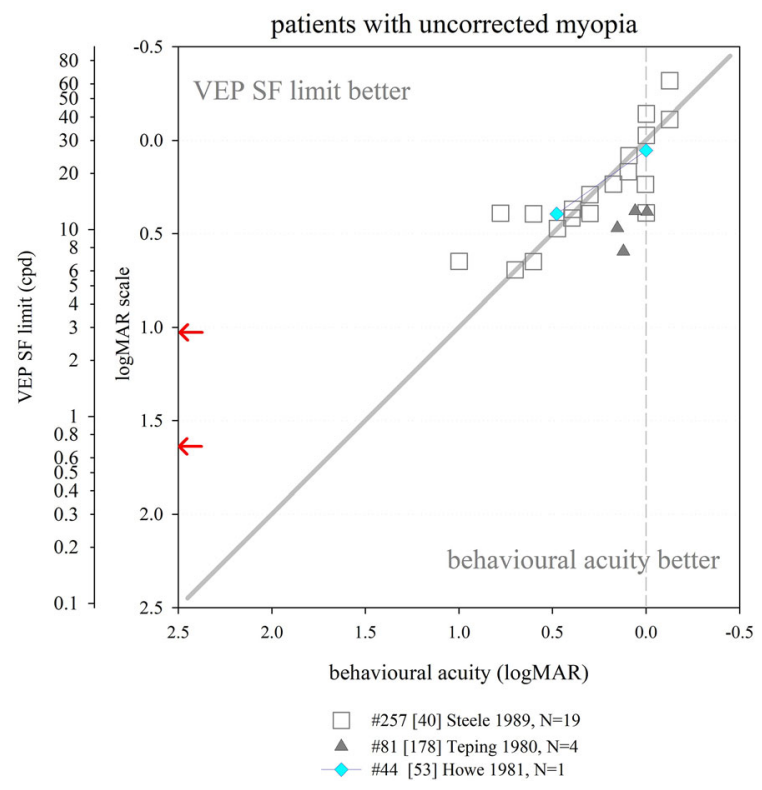

Fig. 11 VEP SF limit versus behavioural acuity in patients with uncorrected myopia. Solid grey line: equality. Dashed vertical grey line: indicative normal behavioural acuity limit (0.0 $\operatorname{logMAR})$. Red arrows at the SF axis indicate the two ISCEV standard checkwidths, $60^{\prime}$ and $15^{\prime}(0.71$ and $2.8 \mathrm{cpd})$ 
whom no VEP SF limits were obtained (VEPs absent, or present only to the largest grating pattern $\left(96^{\prime}\right.$, $0.3 \mathrm{cpd}, 1.98 \log$ MAR)) had behavioural acuities from 1.2 to poorer than 1.6 $\log$ MAR [40]. For four uncorrected myopes, VEP SF limits were closely correlated and on average $0.37 \log$ units poorer than behavioural acuity, but note the relatively dim $\left(10 \mathrm{~cd} \mathrm{~m}^{-2}\right)$, low-contrast $(20 \%)$ VEP stimulus [178]. One adult myope had VEP SF limits of 27 and $12 \mathrm{cpd}$ (0.054 and $0.394 \log$ MAR) with and without refractive correction, respectively, and behavioural acuities of 0.000 and 0.477 [53]. Seemingly similar findings to those above are described for five patients with (corrected) high myopia and no other disorder, with VEP SF limits underestimating behavioural acuity: in four of the five patients, this difference was less than $0.3 \log$ units [172].

Relatively few data were found describing the effect of refractive error on VEP SF limits, and over a more limited acuity range (normal to around 1.0 $\log$ MAR) compared to the "inverse" situation described in Fig. 8, where adults with no or little refractive error are blurred with plus lenses. As far as the two can be compared, they seem to indicate a similar relationship and highlight the importance of accurate refraction for measuring VEP SF limits.

\section{Retinal conditions}

We identified seven studies with data comparing VEP SF limit with behavioural acuity in patients with primarily or solely retinal dysfunction not restricted to the macula (Fig. 12). Eight patients (five with diabetic retinopathy, one with juvenile $\mathrm{X}$-linked retinoschisis, one with central serous retinopathy and one with retinitis pigmentosa) had widely varying differences between VEP SF limits and behavioural acuities, including two with no difference [40]. This pattern of inconsistent agreement was also found for nine further patients, four with retinitis pigmentosa [102], one with diabetic retinopathy [55], two with a rod-cone dystrophy $[55,95]$ and one with a cone abnormality [187]. In a study of 11 patients with either retinitis pigmentosa or choroidal atrophy, behavioural acuity was systematically better than VEP SF limit: however, this study employed a relatively $\operatorname{dim}\left(10 \mathrm{~cd} \mathrm{~m}^{-2}\right)$ and lowcontrast (20\%) VEP stimulus [178]. One further patient, used to illustrate a large discrepancy between VEP SF limit and behavioural acuity associated with

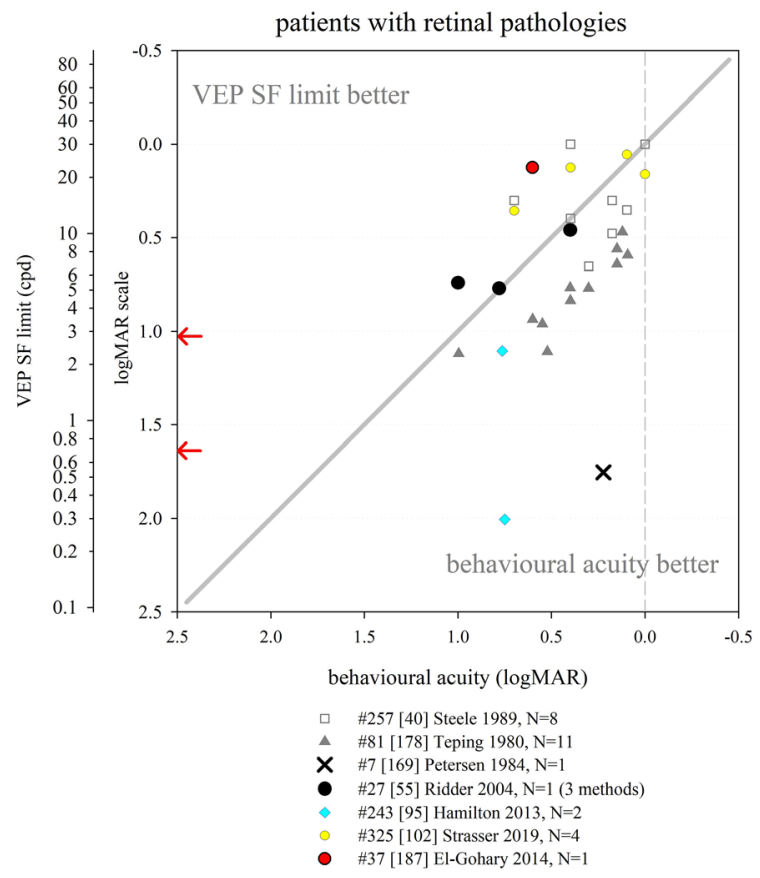

Fig. 12 VEP SF limit versus behavioural acuity in patients with various retinal pathologies. Solid grey line: equality. Dashed vertical grey line: indicative normal behavioural acuity limit (0.0 logMAR). Red arrows at the SF axis indicate the two ISCEV standard checkwidths, $60^{\prime}$ and $15^{\prime}(0.71$ and $2.8 \mathrm{cpd})$

low amplitude VEPs, had mild acuity loss (0.22 $\log$ MAR) but a very poor VEP SF limit (1.75 $\log$ MAR) [169]; a similar discrepancy was also reported in a 6-year-old child with a cone dystrophy [95]. In contrast, in 14 patients with retinal dystrophies, the average VEP SF limit was $0.126 \log$ units better than behavioural acuity [118]. These findings suggest that-similar to patients with opacitiesretinal disease impairs VEP SF limits and behavioural acuities similarly, albeit with wider disparities. The trends seen in these studies of patients with retinal disease are similar to those in healthy, normally sighted adults with artificially reduced acuity using plus lenses or Bangerter foils (Fig. 8). While a retinal pathology may degrade portions of the stimulus, the spatial integration which takes place along the visual pathway may minimise the evidence of localised retinal dysfunction on the VEP. Conversely, preservation of even a small (e.g. 2 degrees) central portion of the fovea may still afford good or even excellent behavioural acuity, but markedly reduce or even eliminate the VEP [169]. A simulation of this effect has been described [29, Fig. 3B]. 


\section{Macular conditions}

We identified 11 studies which compared VEP SF limits with behavioural acuity in patients with macular disease (Fig. 13). Two studies with fairly large groups of patients found a preponderance of cases with VEP SF limits poorer than behavioural acuities: 32/34 patients [188] and $47 / 50$ eyes of 27 patients [189], respectively. Subjects were adult or older children, so behavioural acuity could be considered as gold standard. A smaller study also found poorer VEP SF limits than behavioural acuity in six patients with macular disease; however, this could at least partly be due to the relatively $\operatorname{dim}\left(10 \mathrm{~cd} \mathrm{~m}^{-2}\right)$, low-contrast (20\%) VEP stimulus [178].

Five studies presented individual patients with macular disease with quite closely matched VEP SF limits and behavioural acuities [55, 95, 118, 169, 172], one of which also noted that $28 / 35$ eyes $(80 \%)$ with macular diseases had a visual acuity difference between the two acuities within 1.0 octave $(0.3 \log$

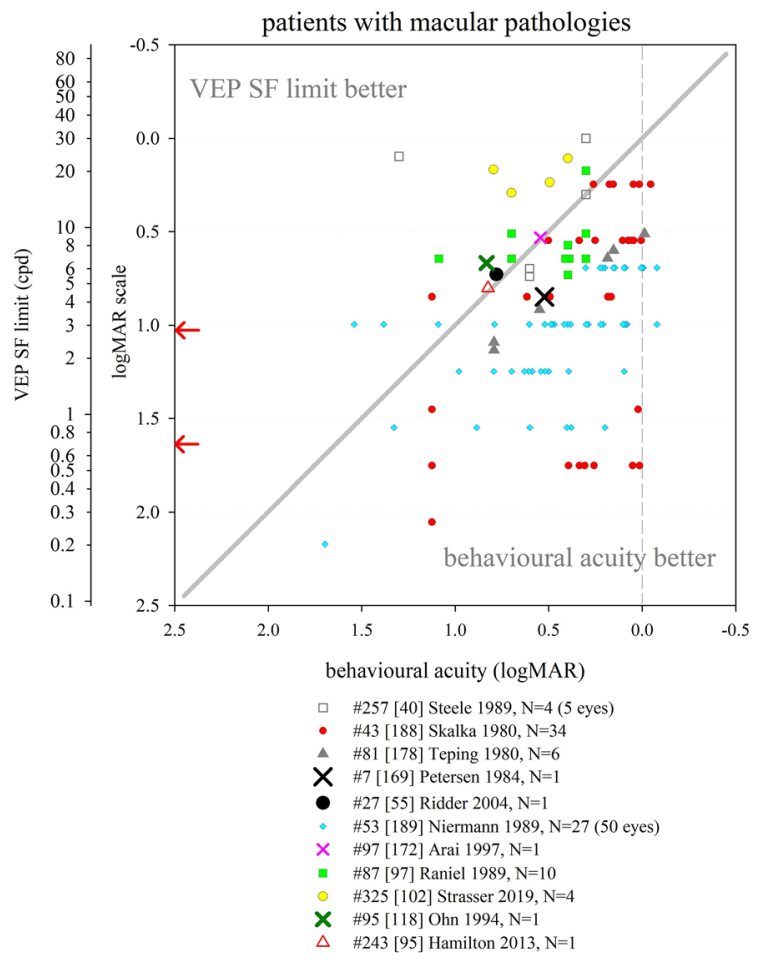

Fig. 13 VEP SF limit versus behavioural acuity in patients with macular pathologies. Solid grey line: equality. Dashed vertical grey line: indicative normal behavioural acuity limit (0.0 $\log$ MAR). Red arrows at the SF axis indicate the two ISCEV standard checkwidths, $60^{\prime}$ and $15^{\prime}$ (0.71 and $\left.2.8 \mathrm{cpd}\right)$ units) (data for the 35 eyes not extractable) [172]. In a large group of 67 patients with macular disease, the average VEP SF limit was only 0.04 log units poorer than behavioural acuities, but showed wide variability: one example patient had a VEP SF limit $0.163 \mathrm{log}$ units better than behavioural acuity [118]. Additional small groups of patients ( $N=4$ and 10 , respectively) with macular pathology were noted to have approximately equal VEP SF limits and behavioural acuities [40, 97]. One study found VEP SF limits better than behavioural acuity in all four patients studied [102].

Macular disease appears predominantly to result in VEP SF limits which are substantially poorer then behavioural acuity, but findings are sufficiently scattered to suggest that any correlation is very weak (Fig. 13). The extent of macula affected may govern the quality of this correlation: where disease affects only the fovea, the rest of the macula may continue to generate a VEP. Experiments with mimicked central scotomas showed a two degree scotoma only slightly affected the VEP SF limit, but VEP SF limits worsened as the scotoma increased in size: VEP amplitude was reduced at all SFs, reducing the slope of the linear amplitude extrapolation [29].

\section{Optic nerve}

We identified 11 studies with extractable data where patients with optic nerve disorders had both VEP SF limits and behavioural acuity compared (Fig. 14). In general, VEP SF limits were poorer than behavioural acuity. In a large group of patients $(N=68)$ with optic nerve disease, an average deficit of around $0.2 \mathrm{log}$ units was noted in the VEP SF limits, but with wide discrepancies: this is illustrated with an example (Fig. 14) which did not follow the group trend, where a patient with behavioural acuity of $0.176 \log$ MAR had an excellent VEP SF limit of $-0.22 \log$ MAR [118]. VEP SF limits poorer than behavioural acuity was found in $22 / 23$ eyes in a group of patients with a variety of optic nerve diseases including retrobulbar neuritis, ischaemic optic neuropathy, traumatic neuropathies and optic nerve tumours [188]; eight patients (four with optic atrophy, four with optic neuritis) had VEP SF limits poorer than behavioural acuity, albeit using a relatively $\operatorname{dim}\left(10 \mathrm{~cd} \mathrm{~m}^{-2}\right)$, low-contrast (20\%) VEP stimulus [178]. Specific examples where VEP SF limits were markedly poorer than behavioural acuity include a child with optic nerve disease but 


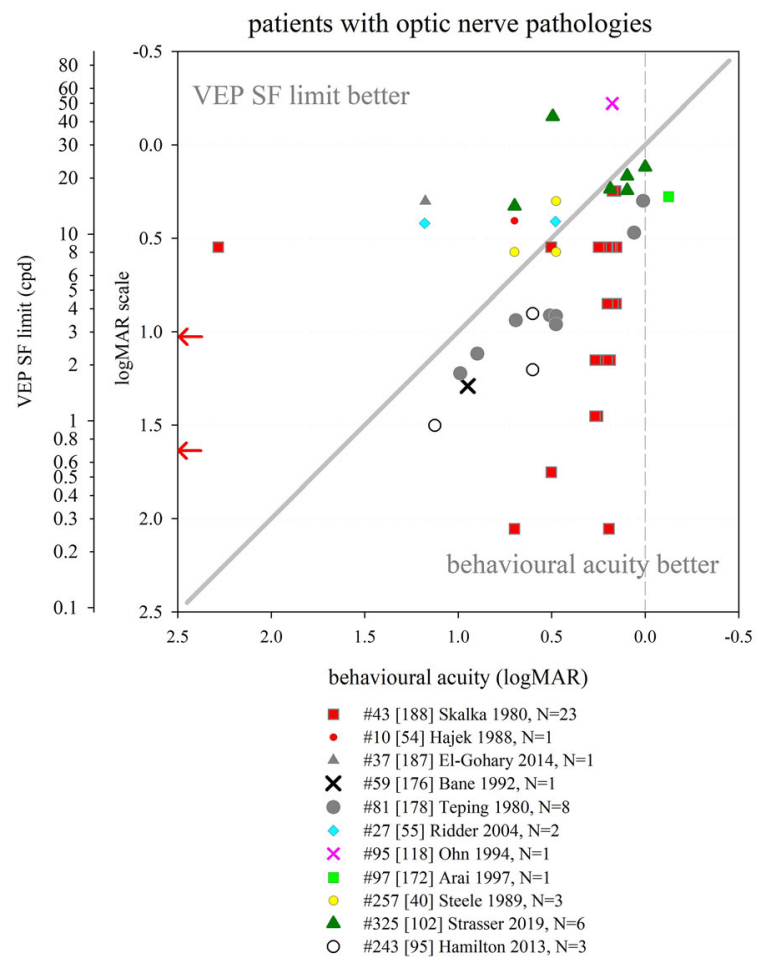

Fig. 14 VEP SF limit versus behavioural acuity in patients with optic nerve pathologies. Solid grey line: equality. Dashed vertical grey line: indicative normal behavioural acuity limit (0.0 logMAR). Red arrows at the SF axis indicate the two ISCEV standard checkwidths, $60^{\prime}$ and $15^{\prime}(0.71$ and $2.8 \mathrm{cpd})$

without nystagmus or seizure disorder [176] and a 47-year-old man with optic neuritis and excellent behavioural acuity $(-0.125 \log$ MAR vs. VEP SF limit of 0.278 ) [172]. The latter study investigated a group of 27 patients with optic neuritis or optic atrophy and found poor correlation of behavioural acuity and VEP SF limit: only 17/27 had a difference within 1.0 octave (0.3 log units) [172]. Mean VEP "scores" (log reduction relative to age-expected norms) of $0.95 \log$ units were worse than behavioural acuity (Teller Acuity cards) "scores" of $0.86 \log$ units in children whose visual impairment included optic nerve atrophy [183].

Examples of closer matching of VEP SF limits and behavioural acuity, or of VEP SF limits better than behavioural acuity were noted in six patients with optic neuritis [102], in three patients, one with optic nerve hypoplasia, one with optic neuritis, and one with toxic optic neuropathy [40], in a patient with optic neuropathy [54], in two patients with optic nerve hypoplasia [55] and in a young (2.5-year old) patient with optic atrophy [187].

Optic nerve disease therefore seems to result in VEP SF limits which are usually, but not always, poorer than behavioural acuity, with evidence of wide scatter (Fig. 14). This may be related to the wellestablished phenomenon of optic nerve disease often reducing VEP amplitude and therefore SNR. Smallbut-extant VEPs close to the SF limit are therefore likely to be mis-categorised as absent, worsening VEP SF limits, a situation which could be improved by employing longer recording times close to the SF limit to enhance SNR: however, most procedures employ fixed recording duration for every pattern size. For extrapolated VEP SF limits, lower amplitudes also lead to flattened spatial tuning functions [118, 172] and hence reduced slope of the linear amplitude extrapolation, increasing the error associated with the crossing point, especially if SF sampling is sparse towards the limit and/or if the SF axis is logarithmically scaled. This might explain the wide discrepancies between VEP SF limit and behavioural acuities noted in optic nerve disease [118, 172, 173, 177].

\section{Amblyopia}

Amblyopia-reduced optotype acuity measured from one or both eyes not exclusively attributed to a structural abnormality of the eye and due to impaired development of a normal cortical visual pathway-has been extensively investigated with VEPs because of the cortical pathway involvement and the need for objective tests at the typical ages of patients. We identified nine studies which measured VEP SF limits in children or adults with amblyopia (Fig. 15). Patients included adults and children. Some had a mixture of treated (patching/surgical) and untreated patients, but most studies did not state whether patients had ever been treated. VEP SF limits demonstrated rapid acuity improvements in two young ( $<2$ years) children due to patching of the better eye, but not in two older children: contemporary behavioural acuities were not measured [190]. Where both VEP SF limits and behavioural acuities were recorded, VEP SF limits were almost always better. VEP SF limits improved from 17 to $20 \mathrm{cpd}$, i.e. 0.257 to $0.180 \log$ MAR 1 month before and after extraction of a subcapsular cataract and fitting of a soft contact lens aphakic correction, while behavioural acuities 


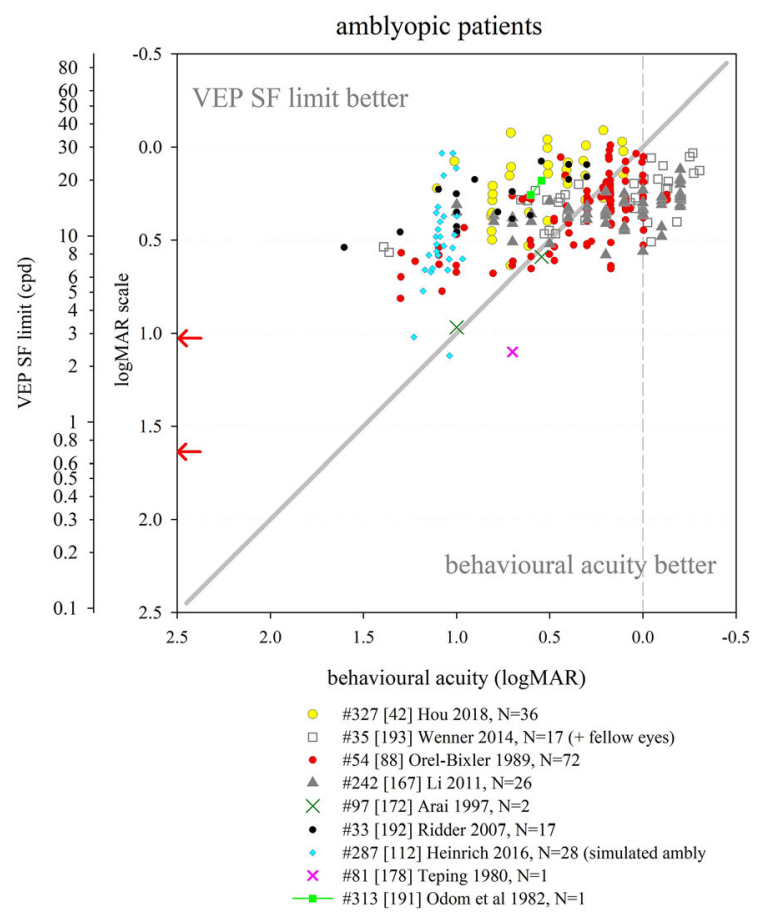

Fig. 15 VEP SF limit versus behavioural acuity in amblyopic patients, some also with fellow eye data. Solid grey line: equality. Dashed grey line: indicative normal behavioural acuity limit $(0.0 \log$ MAR $)$. Red arrows at the SF axis indicate the two ISCEV standard checkwidths, $60^{\prime}$ and $15^{\prime}$ (0.71 and $\left.2.8 \mathrm{cpd}\right)$

improved from 0.602 to $0.544 \log$ MAR [191]. A detailed study of 72 amblyopic patients (2-61 years) with behavioural acuities (Bailey-Lovie chart) ranging from 0.4 to $1.6 \log$ MAR in their amblyopic eye found VEP SF limits were better than optotype acuities: this difference became more marked as acuity worsened, with the VEP SF limit increasingly overestimating optotype acuity [88]. Similarly, VEP SF limits were around $0.5 \log$ MAR units better than behavioural acuities of the poorer eye (0.3-1.6 $\log$ MAR) of 17 pre-treatment amblyopic patients. After 3-20 months of treatment, the 17 patients' behavioural acuities matched their pre-treatment VEP SF limits (5-95th percentiles of difference -0.24 to $0.15 \log$ MAR). The authors concluded that VEP SF limits are a good predictor of post-therapy Snellen acuity [192]. Slightly better VEP SF limits than behavioural thresholds were found for the poorer eye of 26 amblyopic children aged 3 to 12 years: VEP SF limits exceeded behavioural acuities by about $0.1 \mathrm{log}$ units across all acuities, but by much more at poorer acuity [167]. VEP SF limits were also found to be better (by $0.2 \log$ MAR units) overall than behavioural (Landolt $\mathrm{C}$ ) acuity in 17 adult amblyopic patients, and again, the difference was strongly dependent on underlying acuity, with the disagreement larger for poorer acuity [193]. Similarly, while VEP SF limits correlated with behavioural (Bailey-Lovie letter chart) acuity in 26 adult amblyopes, VEP SF limit was almost always better, an effect which was more marked for poorer acuity [42]. One study described two amblyopic patients with behavioural acuities (EDTRS chart, 0.544 and $1.000 \log$ MAR) closely matched to their VEP SF limits (0.591 and 0.968 $\operatorname{logMAR}$ ) [172], and only one study described a poorer VEP SF limit (1.1 logMAR) than behavioural acuity (0.70 logMAR) in an amblyopic adult, using a relatively $\operatorname{dim}\left(10 \mathrm{~cd} \mathrm{~m}^{-2}\right)$, low-contrast $(20 \%)$ VEP stimulus [178].

The evidence from these studies suggest that VEP SF limits are relatively insensitive to the acuity reduction seen in amblyopia when using an optotype-based acuity test, overestimating behavioural acuity markedly for poor optotype acuity, matching acuity at around $0.3-0.5 \log$ MAR, and often underestimating acuity for acuities better than around 0.3 $\log$ MAR, as for normally sighted individuals. A similar mismatch has been extensively described for psychophysically measured grating acuity in amblyopia, while measures of Vernier acuity, either VEPbased or psychophysical, match optotype acuity more closely [194, 195]. A VEP SF limit represents a taskfree threshold to high-contrast, repetitive stimuli and is therefore relatively robust to the higher neural noise and eccentric or unsteady horizontal fixation found in amblyopic vision [55]. VEP grating or checkerboard stimuli are also probably more robust to the "phasescrambling" effect of amblyopia than are optotypes: a study of 27 adults whose vision was degraded to emulate the distorted and fragmented nature of amblyopic vision found VEP SF limits markedly exceeded behavioural thresholds by around 0.58 $\operatorname{logMAR}$ units, while no such overestimation was found for VEP SF limits from vision degraded with simpler blurring with frosted panes [112]. In conclusion, VEP SF limits are not optimal for monitoring amblyopia-associated visual acuity losses since they underestimate optotype-based acuity and are relatively insensitive to optotype-based acuity changes. 


\section{Neurological or structural brain abnormalities}

VEP SF limits are used to infer acuity when neurological or structural brain abnormalities preclude behavioural testing, for example due to speech impairment, inability to point, poor or absent head or trunk control or eye movement disabilities such as gaze apraxia impairing fixation. Patients with these impairments may be mislabelled as visually impaired, and in some cases, VEP assessment may reveal otherwise hidden visual pathway capability. Patients with brain tumours, hydrocephalus, lissencephaly, microcephaly, delayed visual maturation (DVM), cerebral palsy $(\mathrm{CP})$, periventricular leukomalacia (PVL), prematurity sequelae, seizures, or any of neurological disorders such as West syndrome, Aicardi's Syndrome or neuronal ceroid lipofuscinoses may fall into this category.

For the populations in whom relative success has been reported, success rates of establishing VEP SF limits are generally higher than for behavioural methods. Success rates were 56/59 (95\%) versus $41 / 59(69 \%)$ in a group of patients with multiple, diverse neurological and visual disorders, aged 3-33 years; the 15 patients who could not undertake the preferential-looking grating acuity card test had a wide range of VEP SF limits $(0.75-11 \mathrm{cpd}$, i.e. 1.6-0.44 $\log$ MAR), suggesting that the level of vision alone did not predict which test type would be possible [65]. Similarly, 167/173 (97\%) children with CP, aged 6-48 months, provided a VEP SF limit while only 148/173 (85\%) completed Teller Acuity Card testing, even though the behavioural test was undertaken first [196]. Only 54/76 (71\%) children with CP, aged 2-19 years, could be reliably tested with optotypes; children with the most severe motor impairment were most unsuited to optotype testing due to upper motor limb dysfunction and/or speech impairment. VEP SF limits were established in 13 of the $22(59 \%)$ children who could not undertake optotype testing, and failure to record a VEP SF limit was at least partly due to the trunk, head and neck instabilities, nystagmus and gaze apraxias or palsies prevalent in more severe $\mathrm{CP}$ which impair the child's ability to maintain steady fixation on the VEP stimulus screen [197]. Success rates may be poorer when transient rather than steady-state VEPs are used, probably due to the much longer recording times required to find reproducible waveforms at slow reversal rates: 62/75 (83\%) children (5-192 months) with multiple, diverse neurological impairments completed VEP SF limit testing, while 57/75 (76\%) completed behavioural acuity testing [198]. However, VEP SF limits based on transient VEPs were established in 10/11 girls with Rett syndrome, none of whom could complete behavioural testing [199].

VEP SF limits established in 13/22 (59\%) children with CP aged 2-19 years and who could not undertake optotype acuity testing showed a trend of worsening limits with increasing CP:0.30 logMAR to 0.45 $\log$ MAR for level 1 to level 5 (of motor impairment), although non-recordable VEP SF limits could be due to motor dysfunction (trunk, head and neck instabilities, nystagmus, gaze apraxias or palsies) rather than visual impairment [197]. Poorer-than-normal VEP SF limits ("normal" defined by 50 healthy, age-matched subjects) were found in 26 of 37 (70\%) children aged 6-48 months with CP but without ophthalmological complaints and with normal fundi, and were more common in children with more severe motor impairment [200], as described elsewhere [197]. Poorerthan-normal VEP SF limits were also found in 29/30 children (6-108 months) with West syndrome [201].

Where comparisons with behavioural tests were made, reasonable agreement with wide scatter was observed. If a trend was apparent, this generally indicated VEP SF limits better than behavioural acuity at poor acuity levels, with the two measures reaching closer agreement for patients with better acuity (Fig. 16). In 41 patients with multiple, diverse neurological and visual disorders, aged 3-33 years, VEP SF limits slightly exceeded behavioural acuity (forcedchoice, preferential-looking grating acuity card test) at the poorest acuities tested, e.g. VEP SF limit of around 1.0 $\log$ MAR for behavioural acuity of around 1.5 $\log$ MAR. This mismatch lessened as acuity improved [65]. VEP SF limits fell within normal limits for around 40/167 children with CP, aged 6-48 months, with normal fundi and no ocular disease, and showed an improving trend with age. VEP SF limits were $0.208 \log$ MAR better on average than behavioural thresholds (Teller Acuity Cards; preferential-looking, two-alternative, forced-choice, staircase procedure); the VEP-behavioural difference showed no correlation with age. Both VEP SF limits and behavioural acuities were poorer for children with more severe motor impairment: the VEP-behavioural difference was also greater for children with more severe motor impairment. Limits of agreement (5th-95th 


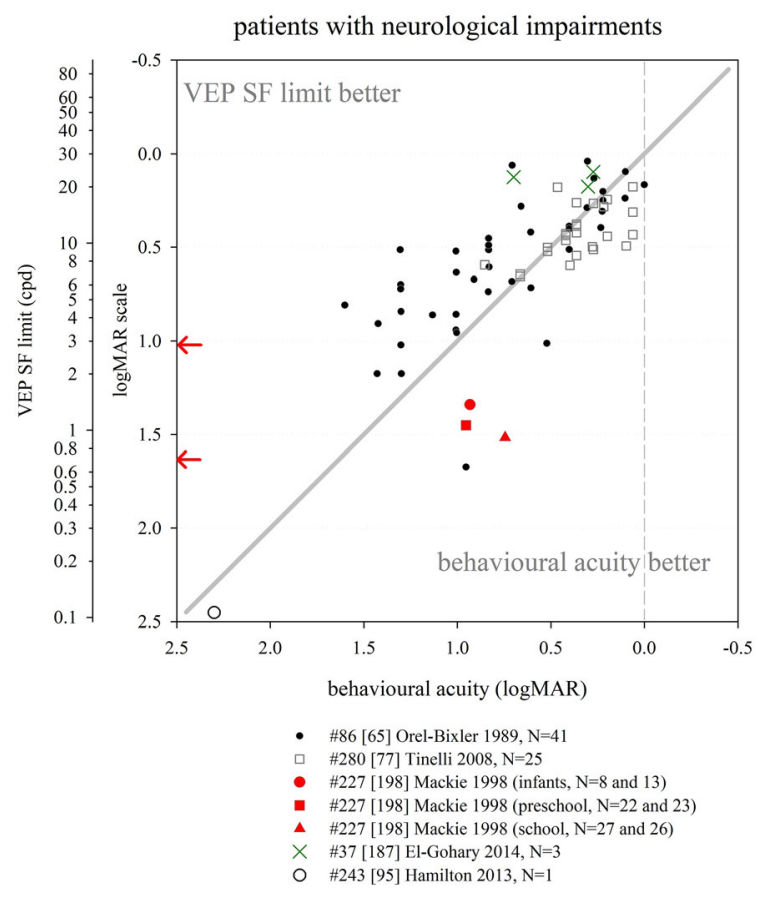

Fig. 16 VEP SF limit versus behavioural acuity in patients with neurological impairments. Solid grey line: equality. Dashed grey line: indicative normal behavioural acuity limit (0.0 logMAR). Red arrows at the SF axis indicate the two ISCEV standard checkwidths, $60^{\prime}$ and $15^{\prime}$ (0.71 and $\left.2.8 \mathrm{cpd}\right)$

percentiles) were $\pm 0.35 \log$ MAR $( \pm 1.2$ octaves $)$ [196]. Very similar VEP SF limits and behavioural acuities (Teller Acuity Cards) were measured in a group of 29 children (9 months to 13 years) with PVL and $\mathrm{CP}$, many of whom were born prematurely and had cognitive impairment: all but one had visual abnormalities. The VEP-behavioural difference tended to increase with age, with little difference on average for children under 1 year, but a tendency towards poorer VEP SF limits than behavioural acuity in older children. Limits of agreement (5th-95th percentiles) for the whole group were $\pm 0.27 \mathrm{log}$ MAR ( \pm 0.9 octaves) [77]. El-Gohary et al. [187] found three patients with CP to have better VEP SF limits than behavioural acuities by around 0.1, 0.2 and $0.6 \log$ MAR units. A 9-year-old child with a craniopharyngioma had equally poor behavioural acuity and VEP SF limit [95].

Poorer VEP SF limits using transient on/offset VEPs without extrapolation (0.78-2.68 logMAR) than behavioural acuities (0.07-2.08 logMAR) were found in a group of 75 children (5-192 months) with multiple, diverse neurological impairments. VEP SF limits did not vary with age, but were poorer in children with a cortical site for the major visual pathway lesion than those with optical, retinal or optic nerve lesion sites. Both acuity measures were poorer in children with more severe motor or intellectual impairment [198].

Reasonable agreement between VEP SF limits and behavioural acuity, expressed as deviation from agetypical values, was found for a large group of paediatric patients with mixed ocular and neurological impairments; $48 \%$ of thresholds agreed within one octave (0.3 logMAR). Children with CP $(N=54)$ had poorer-than-normal VEP SF limits by $0.71 \log$ MAR units on average, while behavioural thresholds were $1.01 \log$ MAR units poorer-than-normal. Children with developmental delay $(N=75)$ had poorer-than-normal VEP SF limits by $0.57 \log$ MAR units on average, while behavioural thresholds were $0.72 \log$ MAR units poorer. Larger deviations from agreement were "contributed to" by the presence of developmental delay, CP or seizures [183].

VEP SF limits for transient VEPs and behavioural acuity were qualitatively described in 100 paediatric patients ( 3 months -8 years) with predominantly neurological impairments: 69/89 were "in agreement", 14/89 (with predominately primary ocular abnormalities) had VEP SF limits which fell short of their behavioural acuity and 6/89 had VEP SF limits which exceeded their behavioural acuity [202].

Cerebral visual impairment (CVI) CVI, recently defined as "a verifiable visual dysfunction which cannot be attributed to disorders of the anterior visual pathways or any potentially co-occurring ocular impairment" [203], can be misdiagnosed as a disorder that is behavioural or psychological in nature. VEP SF limits in patients with CVI have received specific interest and so is discussed separately from more general neurological or structural brain abnormalities. Definitions of CVI in the studies listed below all adhere to the general principle of bilateral acuity loss due to brain lesions, with normal ocular structures and pupillary reactions.

Studies which compared VEP SF limits with agematched control children's VEP SF limits found deficits in all [74, 79, 204] or most [205] of the patients investigated. Test-retest on a subset of 23 patients showed high reliability $\left(r^{2}=0.662\right.$, 


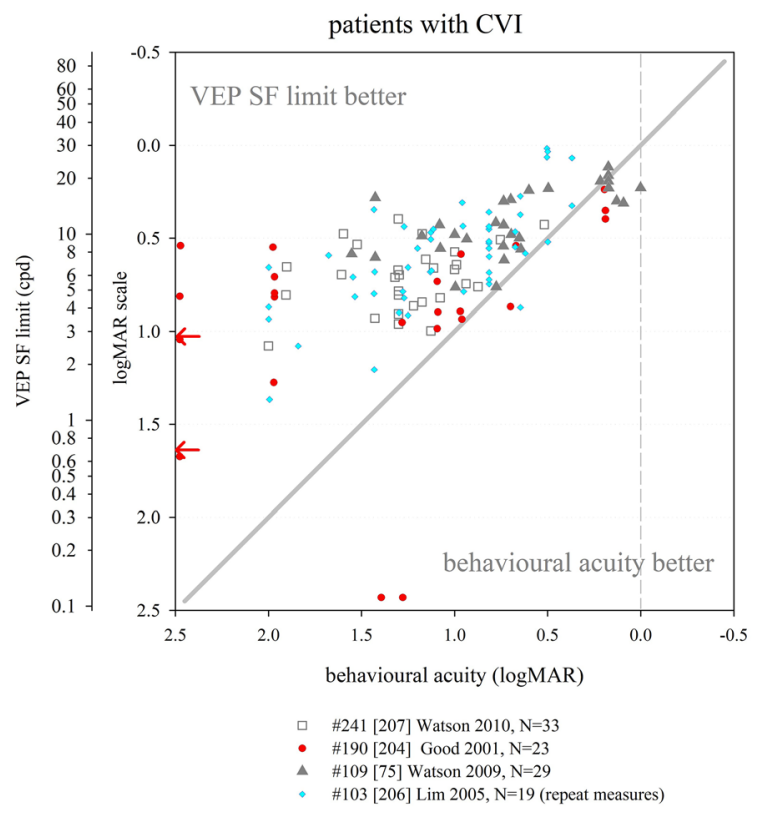

Fig. 17 VEP SF limit versus behavioural acuity in patients with cerebral visual impairment (CVI). Solid grey line: equality. Dashed grey line: indicative normal behavioural acuity limit (0.0 logMAR). Red arrows at the SF axis indicate the two ISCEV standard checkwidths, $60^{\prime}$ and $15^{\prime}$ (0.71 and $\left.2.8 \mathrm{cpd}\right)$

$P=0.0003)$ [204]; slightly better VEP SF limits were noted in CVI children using $\operatorname{dim}\left(20 \mathrm{~cd} \mathrm{~m}^{-2}\right)$ versus bright $\left(109 \mathrm{~cd} \mathrm{~m}^{-2}\right)$ stimuli (10 cpd vs. $\left.7.3 \mathrm{cpd}\right)$, an effect not found in control children [126].

We identified four studies where both VEP SF limits and behavioural acuities were recorded from cohorts of CVI patients (Fig. 17). In all four, VEP SF limits and behavioural acuities were related, albeit with variable levels of agreement. VEP SF limits were generally better than behavioural thresholds, especially at poor acuity levels [75, 204, 206, 207].

Typically, both VEP SF limits and behavioural acuity (preferential-looking) acuity showed equal improvements in CVI patients during the developmental course [206]. It was noted that VEP SF limits are most useful in children with CVI who are difficult to engage or who make no or only fleeting eye contact; the authors also note that VEP SF limits can be astonishingly good for the child's level of visually guided behaviour [206]. VEP SF limits matched closely to behavioural acuity measured 2-13 years later, suggesting a role for "predicting" developed acuity. However, this presumably simply reflects the difference in maturational curves of the two tests, and high variability might be misleading in some instances [207].

\section{Non-organic visual loss}

Non-organic visual loss (NOVL) refers to reduced visual function (here, specifically acuity loss) not caused by any organic lesion. Functional visual loss or medically unexplained visual loss are alternative terms. All three terms avoid assumptions about secondary gain or aetiology, which cannot be established via ophthalmic examination. Patients presenting with NOVL may or may not have voluntary control over their symptoms as they present along a spectrum from malingering/factitious disorders to somatisation/conversion (previously "hysterical") disorders [208].

Thirteen studies were identified where patients with, or suspected of having, NOVL had both behavioural acuity and VEP SF limit documented, 12 with extractable data (Fig. 18). All VEP data are presented in terms of SF limit, either extrapolated or finest SF, rather than in terms of a "corresponding acuity". Even without applying such adjustments, VEP SF limits were almost universally better than behavioural acuity. Two studies with moderately sized groups showed normal VEP SF limits for all patients, despite behavioural acuities as poor as $2.3 \log$ MAR [40, 95]. For 27 children (5-15 years) with NOVL, VEP SF limits were 0.54 (range 0.11-2.79) log units better than behavioural acuity. One further child had visual perceptual difficulties, an optotype acuity of $5 / 12(0.380 \log$ MAR $)$ and VEP SF limit of $15 \mathrm{cpd}$ (within local reference limits), illustrating the inability of VEP SF limits to reflect higher visual processing difficulties [95]. In 28 eyes of 17 patients (7-68 years), VEP SF limits were significantly better than Snellen acuities and the authors noted that VEPs was the method able to "definitively and objectively diagnose functional visual loss" [40]. Similar findings were noted in smaller groups or in individual patients, whether children or adults $[29,54,109,168,169,173$, $178,209]$. The great majority of a large group $(N=100)$ of patients with suspected NOVL were found to have an extant VEP to the finest SF used (5.5' checkwidth, $7.7 \mathrm{cpd}$ ) regardless of contrast. This VEP was evident even at low (20\%) contrast, a finding associated with behavioural acuities of -0.079 to $0.000 \log$ MAR in 10 healthy adults tested 


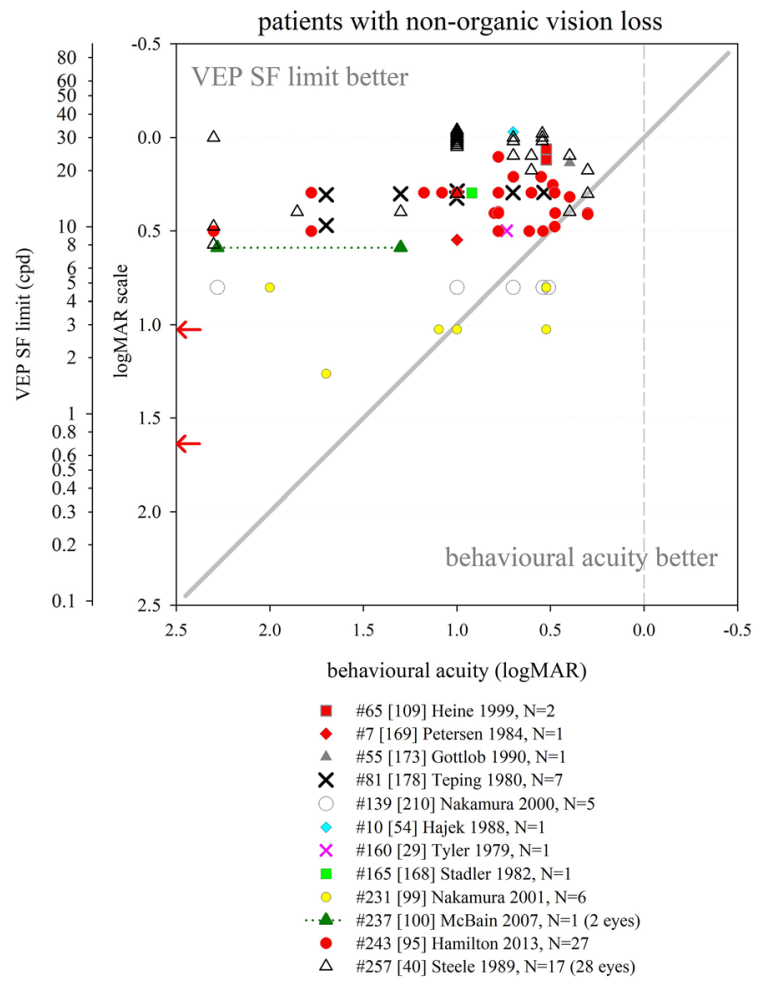

Fig. 18 VEP SF limit versus behavioural acuity in patients with non-organic visual acuity loss. Solid grey line: equality. Dashed grey line: indicative normal behavioural acuity limit (0.0 $\log$ MAR). Red arrows at the SF axis indicate the two ISCEV standard checkwidths, $60^{\prime}$ and $15^{\prime}(0.71$ and $2.8 \mathrm{cpd})$

with the same protocol [100]—illustrative data from both eyes of one patient are shown in Fig. 18.

Two studies found generally poor VEP SF limits but nonetheless concluded that VEP SF limits were in keeping with better behavioural acuities than reported by patients [99, 210]. For five children (7-14 years) suspected to have NOVL with interpretable VEPs [210] and for six adults suspected of malingering [99] all had VEP SF limits (finest SF technique) at a 9', lowcontrast checkwidth (4.7 cpd, $0.804 \log \mathrm{MAR} ; 15 \%)$, which the authors note corresponds to a behavioural acuity of "nearly" $0.000 \log$ MAR.

While all VEP SF limit assessments should be conducted as one part of a full ophthalmic and electrophysiological assessment, this may especially be the case for suspected NOVL. As can be seen from Figs. 12, 13 and 14, normal VEP SF limits might be obtained in the presence of retinal, macular or optic nerve pathologies. Unless all possible organic causes for visual loss have been ruled out by ophthalmic and electrophysiological investigations, interpreting a normal VEP SF limit as confirmation of NOVL could miss sight-threatening pathology.

\section{Other conditions}

VEP SF limits have been described in relatively small numbers of patients with a variety of other ophthalmic conditions not already described.

Strabismus: Fifteen infants (10-50 weeks), otherwise typically developing, with untreated esotropia and alternating fixation had monocular and binocular VEP SF limits which fell 0.30 and $0.23 \mathrm{log}$ units, respectively, below the averages for age-matched control infants, but they did not have significant interocular VEP SF limit differences [211]. Four 2-year olds with strabismus and alternating fixation but without amblyopia were found to have VEP SF limits (0.68-0.13 logMAR) which quite closely matched behavioural acuities measured several months later. The authors concluded that VEP SF limits accurately predicted future recognition acuity [186].

Glaucoma: In seven patients with open-angle glaucoma, VEP SF limits and behavioural acuities showed very good correlation: the difference was within $0.30 \log$ units for $6 / 7$ patients. One example patient, a 40-year-old man with optic nerve head cupping, had behavioural acuity of $0.000 \log$ MAR and a VEP SF limit of $16.1 \mathrm{cpd}(0.27 \log \mathrm{MAR})$. [172]. In 12 patients with glaucoma, those with intact fields ( $N=5,8$ eyes) showed close correlation between VEP SF limit and behavioural acuity: however, if visual field defects were evident $(N=4)$, no VEPs were evident even to the coarsest SF ( $96^{\prime}$ grating, i.e. $0.31 \mathrm{cpd}, 1.98 \log \mathrm{MAR})$ despite behavioural acuities between 0.18 and $0.48 \operatorname{logMAR}$ [40].

Albinism: In 13 children aged $0.1-10$ years with albinism and foveal hypoplasia (11 also with nystagmus), VEP SF limits using horizontal, sinusoidal gratings ranged from 0.176 to $1.176 \log \mathrm{MAR}$, generally poorer than typical for age (cf Fig. 6) [212]. Two patients with albinism had better VEP SF limits than behavioural acuities by around 0.1 and $0.3 \log$ units [187]. An 8-year-old boy with albinism including nystagmus had behavioural acuity of $1.000 \log$ MAR and VEP SF limits about $0.1 \log$ units better [173], and a 3-year-old patient with albinism had a VEP SF limit $0.08 \log$ units better than behavioural acuity [55]. 
Limited data on the presence or absence of foveal hypoplasia and/or nystagmus, and grouping of data across ages when VEP-behavioural differences are likely to change markedly, preclude drawing conclusions about VEP SF limits in albinism.

Down syndrome: Researchers established a VEP SF limit in 16/28 (57\%) of young children with Down syndrome and in $91 \%$ of age-matched control children. VEP SF limits and behavioural acuities were 0.2-0.3 log units poorer in children with Down syndrome, not entirely attributable to attention or accommodation effects, suggesting a primary sensory deficit [158].

Autistic spectrum disorder (ASD): Sixteen children (5-17 years) with ASD, no learning disability and corrected-to-normal visual acuity had the same VEP SF limits as an age-matched control group (24.6 vs. $25.8 \mathrm{cpd} ; 0.086$ vs. $0.066 \log \mathrm{MAR})$. The ssVEP second harmonic in children with ASD was smaller across mid-range SFs (5-17 cpd), especially at the right occipital electrode, which the authors suggested reflects compromise of a highly specific neural substrate early in the visual pathway [72].

Vigabatrin-treated infantile spasms: VEP SF limits were investigated in a group $(N=42)$ of children with infantile spasms using, or who had used, vigabatrin. Presence of vigabatrin-related retinal toxicity was presumed if two or more consecutive flicker ERGs had reduced age-corrected amplitude relative to baseline and relative to previous recording by more than the inter-visit variability. VEP SF limits were poorer in those with presumed retinal toxicity $(N=10)$ than in those without $(N=32)$ : 0.42 versus $0.27 \operatorname{logMAR}$. Expressed relative to mean VEP SF limits of agematched controls, children with presumed retinal toxicity had poorer VEP SF limits by $0.144 \log$ units, while those without had better limits by $0.032 \log$ units [213].

\section{Discussion}

It is clear from the large body of literature systematically reviewed here that the VEP SF limit has been applied widely but has yet to be extensively accepted as an objective acuity estimator. One reason for this may be the lack of a standardised protocol and hence, in some cases, widely disparate findings from different laboratories. Another reason is the difficulty of interpretation: for example, what does a VEP SF limit of $10 \mathrm{cpd}$ mean? The findings presented here clearly indicate that it does not mean the same thing in an adult with cataract as in a child with optic nerve hypoplasia or a baby with poor visual behaviour.

The International Society for Clinical Electrophysiology of Vision (ISCEV) writes and updates standards [66, 214-217], guidelines [218, 219] and extended protocols [220-224] with the aim of reducing inter-laboratory test variability, one aspect of quality improvement which reduces inherent uncertainty and enhances patient safety. The ISCEV standards, guidelines and extended protocols address quality of the test process, provide some guidance on appropriate clinical use, and address some aspects of interpretation and communication of test results. An extended protocol ("specifications for specialized procedures that are sufficiently well established and that have broad acceptance by experts in the field") for estimating acuity with VEPs is in press [225].

The challenge associated with VEP SF limit interpretation has its origins in psychophysics, where traditionally a threshold stimulus with a fundamental SF of $30 \mathrm{cpd}$ has been associated with a visual acuity of $0.000 \log \mathrm{MAR}$, i.e. 1.0 (decimal), $6 / 6$ or $20 / 20$ (Snellen). This relationship often fails to hold for VEP SF limits, as illustrated in Figs. 5 and 7. We conclude that it is misleading and inaccurate to arithmetically convert the fundamental SF of the limiting VEP stimulus into the units and terminology of perceptual (behavioural) acuity as this would imply a direct relationship which is accurate in only limited cases. For the same reason, we have reservations about the expression "VEP acuity" because of the risk that nonexpert clinical users will expect the same capability of patients as they would from letter acuity. Retaining units of cpd and establishing reference values and critical limits in those units for clinical reports might circumvent this issue. An alternative approach is to quantify the empirical relationship between an optimised VEP SF limit technique and psychophysical (or behavioural) acuity results: certainly in adult patients tested with robust methodology, there is good evidence for the validity of this approach. However, this empirical calibration is highly dependent on age, VEP technique and-especially for paediatrics-acuity test. Furthermore, the relationship between VEP SF limit and behavioural acuity obtained from artificially blurred, healthy adults (Fig. 8) does not necessarily 
accurately emulate findings from patients with visual acuity loss (e.g. Fig. 9), although Fig. 9 reflects many diverse methodologies, not all necessarily robust. Precision is also an issue: where this has been assessed, the limits of agreement between VEP SF limit and behavioural acuity, even when calibrated empirically, are typically $\pm 0.3 \log$ units ( \pm 1 octave) and can be much wider. This precision should be established via reference values and quoted and interpreted in clinical reports.

Assuming adequate technical reporting of a VEP SF limit, including its age-specific accuracy and precision, clinical interpretation presents a further challenge. For reasons outlined in the introduction, a VEP SF limit has not assessed the same aspect of vision as a clinical acuity test. Good examples of wording which avoids the misleading impression that the VEP SF limit equates to behavioural acuity include: "the presence of a response to a pattern stimulus implies that the visual system contains elements capable of resolving the stimulus" [117]. In conjunction with an empirical calibration, "with [X\%] likelihood the visual resolution measured from early cortical visual processing corresponds to an acuity of better than [value in, e.g. logMAR]" [12]. The latter example also notes the possibility of an organic disorder affecting higher visual areas or NOVL.

The data collated here from studies of patients with visual acuity loss suggest that VEP SF limits are a good proxy for behavioural acuity in several conditions, where "good" is defined as accurate over a range of acuities, with reasonable precision. Such conditions include patients with media opacities, refractive errors and primarily retinal dysfunction.

Where the primary site of dysfunction is the macula, the optic nerve or any cerebral structures, VEP SF limits have poorer accuracy and precision when compared to behavioural measures. In macular disease, the foveal dominance of the VEP is evident in mostly poor VEP SF limits, while the wide scatter presumably reflects general reduction of VEP amplitudes, giving rise to large errors in extrapolated VEP SF limits (Fig. 13). Optic nerve disease similarly presents VEP SF limits poorer than behavioural acuity with wide scatter, again perhaps due to generally low VEP amplitudes impairing limits and increasing error (Fig. 14).
A large volume of data from amblyopic patients confirm that VEP SF limits are relatively insensitive to reduced optotype acuity in amblyopia-many patients with behavioural acuities as poor as $1.0 \operatorname{logMAR}$ continue to show VEP SF limits similar to those of patients with near-normal acuity (Fig. 15). Because the VEP can define a task-free SF threshold which is relatively robust to higher neural noise and to fixation problems [55], it has been used as a predictive marker for outcomes following therapy: however, VEP SF limits prior to amblyopia therapy, being typically better than behavioural acuity, may simply roughly coincide with later, improved levels of optotype acuity. Diagnostically, VEP SF limits are poorly sensitive to amblyopic acuity loss; therefore, a poor VEP SF limit may indicate incorrect refraction, or subtle macular or optic nerve pathology. Interestingly, the pattern of VEP SF limit versus behavioural acuity seen in amblyopic patients is similar, albeit less extreme, to that seen in children with neurological causes for their vision loss, especially CVI (Figs. 16, 17), i.e. VEP SF limits are generally better than behavioural acuity, with the difference more apparent at poor acuities. This not only reinforces the neurological nature of amblyopia, but also raises the issue of which test should be regarded as the gold standard. For some children with neurological impairment, the additional burden of recognition and motor responses required by behavioural tests might mean the VEP SF limit represents their visual threshold more accurately than an acuity card test based on preferential-looking. In contrast, for children with amblyopia, VEP SF limits may be an inaccurate reflection of their visual capabilities because VEP gratings or checkerboards are relatively robust to the "phase-scrambling" effect.

Non-organic vision loss (NOVL) has received much attention from clinicians working with VEP SF limits, and the data compiled here support its use in this area (Fig. 18). The importance of age-specific reference data with pre-specified accuracy and precision is particularly relevant since some workers state the VEP SF limit to be normal even when the finest SF assessed is relatively large [99, 210], and when similar VEP SF limits have been reported in the presence of retinal, macular or optic nerve pathologies (cf Figs. 12, 13, 14). Unless all possible organic causes for visual loss have been ruled out by ophthalmic, neurological, imaging and electrophysiological investigations, interpreting a within-reference-limits VEP 
SF limit as confirmation of NOVL could miss sightthreatening pathology. Furthermore, a normal VEP SF limit in combination with poor acuity may indicate dysfunction of higher visual processing areas rather than NOVL; such patients may benefit from eventrelated potential threshold measures [226]. In a very small number of extreme cases, patients with no behavioural vision at all can present with extant and even normal pattern VEPs [202, 227].

Perhaps the highest utility of VEP SF limits, other than in NOVL, lies in paediatric testing, whether in pre-verbal children or children with motor or learning impairments which prevent reliable measurement of behavioural acuity. As with all paediatric tests, the diagnostic power of a VEP SF limit depends heavily on adequate, age-stratified, reference data from typically developing infants and children [228]. The data presented here indicate that typical limits increase from 1 to $20 \mathrm{cpd}$ over the first year of life, then increase more slowly to reach adult levels between 2 and 10 years of age (Fig. 6). Success rates are variable, but are better for shorter test protocols. The most marked difference between paediatric and adult VEP SF limits is seen when they are compared with behavioural acuities: as reported by many authors, VEP SF limits are much better than behavioural acuity in the youngest, typically developing infants, but the reverse is found from around 3-5 years, with behavioural acuity being somewhat better than VEP SF limits, as seen in adults. This makes it critical that any empirical calibration of VEP SF limits is established for all ages, and that adult calibrations are not applied to infants or to children younger than around 3-5 years. In a typical adult, a reasonable degree of association between the VEP SF limit and the perceptual threshold is to be expected; in most cases, the resolution threshold for the optical system and visual pathways is similar and perception and attention are not limiting factors. The size of the VEP generated in the occipital cortex reduces approaching the SF limit, representing diminished cortical activation, and extrapolation to zero or to noise indicates absence of a cortical signal and thus a threshold for the entire visual system. The story is different during development: although infant visual sensitivity is limited by optical, photoreceptor, foveal and neural immaturity [229], these do not fully account for the fact that VEP SF the SF limits are far better than behavioural acuity in the first months of life. Two possible reasons have been suggested [230]. Firstly, signals encoding high SFs might be available at the visual cortex to be tapped by the VEP, but then be altered or lost in higher processing centres and therefore unavailable to be tapped by behavioural tests. Secondly, some small signals may be detectable as a VEP following repetitive stimulation and averaging but in behavioural tests, an infant's response is required trial-bytrial with no opportunity for summing stimuli. There is no accepted gold standard technique for measuring infant visual acuity, and both behavioural and electrophysiological measures are flawed. Preferentiallooking tests are neurally demanding and require motor responses which may depress thresholds; however, it is not justifiable to assume an infant can "see" a stimulus which evokes a VEP but which does not elicit a behavioural response. On the other hand, if the VEP SF limit is within the reference range for age and protocol, it is reasonable to infer that the visual pathway from optics to cortex is intact. This holds true regardless of the patient's age (although it may be less true for certain pathologies). For this reason, VEP SF limits, and indeed VEPs, are uniquely valuable for assessing the integrity of the early visual pathway.

Regardless of how a patient presents, a VEP SF limit cannot stand alone, but must be ordered and interpreted in the light of the neonatal, ophthalmological, neurological and neuroradiological, imaging and electrophysiological context of each patient, and with a full understanding of the implications of the VEP protocol including age-specific reference data. The importance of using VEP SF limits as only part of a fuller assessment cannot be overstressed. VEP SF limits cannot be interpreted without full clinical assessment and history; assessment should often include standard clinical electrophysiological testing (full field, flash and/or pattern ERGs, VEPs, etc.), ocular and neural imaging techniques and other diagnostic testing. Its clinical use should be reserved exclusively for patients who cannot or will not cooperate or satisfactorily complete behavioural acuity tests or whose cooperation is suspect: behavioural tests have real-life meaning and are almost always the gold standard. An exception might be if the clinician understands why the VEP SF limit and behavioural acuity might differ, but seeks their complementary information.

Visual electrophysiology laboratories without specific thresholding procedures but which record 
ISCEV standard VEPs to checkwidths of $60^{\prime}$ and $15^{\prime}$ ( 0.71 and $2.8 \mathrm{cpd}$ ), and often to additional pattern sizes, may attribute an acuity according to the presence or absence of these transient VEPs. For example, the statement "good visual acuity means good sized response to smallest checks $\left(6.25^{\prime}\right)$ " was included in a recent consensus statement, in the context of whether a baby or very developmentally delayed child could see [231]. A checkwidth of $6.25^{\prime}$ has $\mathrm{SF}_{\mathrm{f}}$ of $6.8 \mathrm{cpd}$ : an extant VEP (transient or steady state) to this SF would certainly be in keeping with "good visual acuity" for a baby, but would be poorerthan-typical for any patient aged over 1 year. It is normal for subjects aged over a few weeks to have a VEP to the ISCEV standard $60^{\prime}(0.71 \mathrm{cpd})$ checkwidth, and normal for subjects over 6 months to have a VEP to the ISCEV standard $15^{\prime}(2.8 \mathrm{cpd}$ ) (cf Fig. 6) $[232,233]$. Depending on the diagnostic question, one strategy might be to record both a rapid, objective, ssVEP SF limit and transient VEP(s) to pattern sizes informed by the VEP SF limit, or vice versa. This strategy is short enough to have a reasonable chance of maintaining patient engagement while also capturing the rich diagnostic information in the parameters and waveshapes of transient VEPs. For other patients, prioritising transient VEPs, including monocular testing, may be the better strategy to make best use of limited cooperation and attention, and using the VEP SF limit as a valuable adjunct or separate assessment.

This systematic review has several shortcomings. We have undertaken little quality or bias assessment of included studies, principally because no standard outcome measure or intervention was being reviewed. The included studies had widely divergent purposes: many did not address either of the questions of this review as their principal aim. We have indicated as far as possible factors such as number of subjects or patients included. A further shortcoming is the great variety of stimulus, recording and analysis techniques and combinations employed in the included studies, so the effect of altering one parameter such as age is always confounded in other studies by multiple other differences.

The future for VEP SF limits in the clinical setting is promising. Hugely increased, inexpensive computational power has enabled multiple improvements to even the most robust and accurate techniques described here. For example, significance testing and threshold extrapolation could be performed in real time with stopping rules to minimise test duration and to give feedback control for subsequent stimulus presentation [92, 234]. Increased dwell time in low SNR conditions could enhance accuracy and precision of VEP SF limits. SsVEPs are extensively used in the field of brain-computer interfaces (BCI), where accuracy and speed of detection-information transfer rate in BCI language - are essential to reduce user frustration. Principal component analysis, independent component analysis and canonical correlation analysis are widely used for signal detection in BCI [235, 236], but not so far in the field of VEP SF limits. Combining eye tracking with ssVEPs is providing more robust user navigation and takes advantage of the reducing costs and current rapid evolution of both EEG and eye tracking data acquisition components [237]. Eye tracking combined with VEP SF thresholding could automatically restrict analysis to only those EEG epochs captured when the patient is looking at the stimulus, or could use gaze-deviation to trigger an attention-grabbing change of stimulus or sound to help re-establish fixation. Machine learning has already been demonstrated to improve VEP SF limit estimation [238], an approach which could help bypass choices of threshold definition and calibration, and could even use individual sweeps to obviate the need for feature extraction. Finally, event-related potentials [226] and fixation- or saccade-related potentials [239] could bridge the gap between cortical and cognitive SF limits.

Acknowledgements The authors acknowledge Michael Bradnam who, with Ruth Hamilton, Michael Bach and Herbert Jägle, obtained approval from the ISCEV Director of Standards to develop an extended protocol for estimating acuity with VEPs, which triggered this systematic review.

Author contributions RH conceptualised the study; all authors designed the study, $\mathrm{RH}$ designed the data collection instrument, RH \& JVO performed the database searches, All authors screened abstracts and full text for inclusion, All authors collected data, RH coordinated and supervised data collection, RH drafted the initial manuscript, All authors substantially contributed to writing, and critically reviewed and revised the manuscript, and All authors approved the final manuscript as submitted and agree to be accountable for all aspects of the work.

Funding No funding was received. 


\section{Compliance with ethical standards}

Conflict of interest Micahel Bach has consulted for Diagnosys LLC, Lowell, MA, USA.

Informed consent As this article does not contain any studies with human participants performed directly by any of the authors, the concept of informed consent is not applicable.

Statement of human rights The study involved no research on human participants and consent is not applicable.

Statement on the welfare of animals The study involved no research on animals.

Open Access This article is licensed under a Creative Commons Attribution 4.0 International License, which permits use, sharing, adaptation, distribution and reproduction in any medium or format, as long as you give appropriate credit to the original author(s) and the source, provide a link to the Creative Commons licence, and indicate if changes were made. The images or other third party material in this article are included in the article's Creative Commons licence, unless indicated otherwise in a credit line to the material. If material is not included in the article's Creative Commons licence and your intended use is not permitted by statutory regulation or exceeds the permitted use, you will need to obtain permission directly from the copyright holder. To view a copy of this licence, visit http://creativecommons.org/licenses/by/4.0/.

\section{References}

\section{Hashtag numbers indicate the 155 references whose data were included in the systematic review}

1. Harter M, White C (1968) Effects of contour sharpness and check-size on visually evoked cortical potentials. Vis Res 8:701-711

2. Regan D (1973) Rapid objective refraction using evoked brain potentials. Invest Ophthalmol Vis Sci 12:669-679

3. Williams DR (1985) Aliasing in human foveal vision. Vis Res 25:195-205. https://doi.org/10.1016/00426989(85)90113-0

4. Armington JC (1968) The electroretinogram, the visual evoked potential, and the area-luminance relation. Vis Res 8:263-276. https://doi.org/10.1016/0042-6989(68)90014$\mathrm{X}$

5. Heinrich SP, Krüger K, Bach M (2010) The effect of optotype presentation duration on acuity estimates revisited. Graefes Arch Clin Exp Ophthalmol 248:389-394. https://doi.org/10.1007/s00417-009-1268-2

6. Campbell FW, Maffei L (1981) The influence of spatial frequency and contrast on the perception of moving patterns. Vis Res 21:713-721. https://doi.org/10.1016/00426989(81)90080-8

7. Moher D, Liberati A, Tetzlaff J et al (2009) Preferred reporting items for systematic reviews and meta-analyses: the PRISMA statement. PLoS Med 6:e1000097. https:// doi.org/10.1371/journal.pmed.1000097

8. WebPlotDigitizer-Extract data from plots, images, and maps. https://automeris.io/WebPlotDigitizer/. Accessed 4 Jul 2019

9. Kelly DH (1976) Pattern detection and the two-dimensional Fourier transform: flickering checkerboards and chromatic mechanisms. Vis Res 16:277-287. https://doi. org/10.1016/0042-6989(76)90111-5

10. Fahle M, Bach M (2006) Origin of the visual evoked potentials. In: Heckenlively J, Arden G (eds) Principles and practice of clinical electrophysiology of vision. MIT Press, Cambridge, pp 207-234

11. Regan D (1966) Some characteristics of average steadystate and transient responses evoked by modulated light. Electroencephalogr Clin Neurophysiol 20:238-248. https://doi.org/10.1016/0013-4694(66)90088-5

12. Bach M, Maurer JP, Wolf ME (2008) Visual evoked potential-based acuity assessment in normal vision, artificially degraded vision, and in patients. Br J Ophthalmol 92:396-403. https://doi.org/10.1136/bjo.2007.130245 (\#321)

13. Chan H, Odom JV, Coldren J et al (1986) Acuity estimated by visually evoked potentials is affected by scaling. Doc Ophthalmol 62:107-117 (\#214)

14. Allen D, Bennett PJ, Banks MS (1992) The effects of luminance on FPL and VEP acuity in human infants. Vis Res 32:2005-2012 (\#178)

15. Odom JV, Maida TM, Dawson WW, Romano PE (1983) Retinal and cortical pattern responses: a comparison of infants and adults. Am J Optom Physiol Opt 60:369-375 (\#168)

16. Hoffmann MB, Brands J, Behrens-Baumann W, Bach M (2017) VEP-based acuity assessment in low vision. Doc Ophthalmol 135:209-218. https://doi.org/10.1007/ s10633-017-9613-y (\#322)

17. Pirchio M, Spinelli D, Fiorentini A, Maffei L (1978) Infant contrast sensitivity evaluated by evoked potentials. Brain Res 141:179-184. https://doi.org/10.1016/00068993(78)90628-5 (\#314)

18. Ridder WH, Waite BS, Melton TF (2014) Comparing Enfant and PowerDiva sweep visual evoked potential (sVEP) acuity estimates. Doc Ophthalmol 129:105-114. https://doi.org/10.1007/s10633-014-9457-7 (\#246)

19. Hemptinne C, Liu-Shuang J, Yuksel D, Rossion B (2018) Rapid objective assessment of contrast sensitivity and visual acuity with sweep visual evoked potentials and an extended electrode array. Invest Ophthalmol Vis Sci 59:1144-1157. https://doi.org/10.1167/iovs.17-23248 (\#324)

20. Suter S, Suter PS, Deegan J (1990) Steady-state VEP phase-stability and acuity in adults and infants. Clin Vis Sci 5:71-80 (\#88)

21. Lauritzen L, Jørgensen MH, Michaelsen KF (2004) Testretest reliability of swept visual evoked potential measurements of infant visual acuity and contrast sensitivity. Pediatr Res 55:701-708. https://doi.org/10.1203/01.PDR. 0000113769.44799 .02 (\#234)

22. Yadav NK, Almoqbel F, Head L et al (2009) Threshold determination in sweep VEP and the effects of criterion. 
Doc Ophthalmol 119:109-121. https://doi.org/10.1007/ s10633-009-9177-6 (\#150)

23. Allen D, Tyler CW, Norcia AM (1996) Development of grating acuity and contrast sensitivity in the central and peripheral visual field of the human infant. Vis Res 36:1945-1953 (\#224)

24. Norcia AM, Tyler CW, Hamer RD (1988) High visual contrast sensitivity in the young human infant. Invest Ophthalmol Vis Sci 29:44-49 (\#256)

25. Nelson JI, Kupersmith MJ, Seiple WH et al (1984) Spatiotemporal conditions which elicit or abolish the oblique effect in man: direct measurement with swept evoked potential. Vis Res 24:579-586 (\#124)

26. Skoczenski AM, Norcia AM (1999) Development of VEP Vernier acuity and grating acuity in human infants. Invest Ophthalmol Vis Sci 40:2411-2417 (\#228)

27. Zemon V, Hartmann EE, Gordon J, Prünte-Glowazki A (1997) An electrophysiological technique for assessment of the development of spatial vision. Optom Vis Sci 74:708-716 (\#267)

28. Vesely P (2015) Contribution of sVEP visual acuity testing in comparison with subjective visual acuity. Biomed Pap Med Fac Univ Palacky Olomouc Czech Repub 159:616-621. https://doi.org/10.5507/bp.2015.002 (\#39)

29. Tyler CW, Apkarian P, Levi DM, Nakayama K (1979) Rapid assessment of visual function: an electronic sweep technique for the pattern visual evoked potential. Invest Ophthalmol Vis Sci 18:703-713 (\#160)

30. Ridder WH, McCulloch D, Herbert AM (1998) Stimulus duration, neural adaptation, and sweep visual evoked potential acuity estimates. Invest Ophthalmol Vis Sci 39:2759-2768 (\#268)

31. Furuskog P, Wanger P (1986) Visual acuity measurement using evoked potentials and fast Fourier transform. Acta Ophthalmol (Copenh) 64:352-355 (\#9)

32. Norcia AM, Tyler CW, Hamer RD (1990) Development of contrast sensitivity in the human infant. Vis Res 30:1475-1486 (\#89)

33. Norcia AM, Tyler CW (1985) Spatial frequency sweep VEP: visual acuity during the first year of life. Vis Res 25:1399-1408 (\#8)

34. Simon F, Rassow B (1986) Retinal visual acuity with pattern VEP normal subjects and reproducibility. Graefes Arch Clin Exp Ophthalmol 224:160-164 (\#173)

35. Ridder WH, Tong A, Floresca T (2012) Reliability of acuities determined with the sweep visual evoked potential (sVEP). Doc Ophthalmol 124:99-107. https://doi.org/10. 1007/s10633-012-9312-7 (\#205)

36. Almoqbel FM, Irving EL, Leat SJ (2017) Visual acuity and contrast sensitivity development in children: sweep visually evoked potential and psychophysics. Optom Vis Sci 94:830-837. 0000000000001101 (\#326)

37. Almoqbel FM, Yadav NK, Leat SJ et al (2011) Effects of sweep VEP parameters on visual acuity and contrast thresholds in children and adults. Graefes Arch Clin Exp Ophthalmol 249:613-623. https://doi.org/10.1007/ s00417-010-1469-8 (\#111)

38. Spekreijse H (1983) Comparison of acuity tests and pattern evoked potential criteria: two mechanisms underly acuity maturation in man. Behav Brain Res 10:107-117 (\#213)
39. Regan D (1978) Assessment of visual acuity by evoked potential recording: ambiguity caused by temporal dependence of spatial frequency selectivity. Vis Res 18:439-443 (\#120)

40. Steele M, Seiple WH, Carr RE, Klug R (1989) The clinical utility of visual-evoked potential acuity testing. Am J Ophthalmol 108:572-577 (\#257)

41. Douthwaite WA, Jenkins TC (1987) Visual acuity prediction using the visual evoked response. Ophthalmic Physiol Opt 7:421-424 (\#49)

42. Hou C, Good WV, Norcia AM (2018) Detection of amblyopia using sweep VEP vernier and grating acuity. Invest Ophthalmol Vis Sci 59:1435-1442. https://doi.org/ 10.1167/iovs.17-23021 (\#327)

43. Teping C (1981) Determination of visual acuity by the visually evoked cortical potential (author's transl). Klin Monbl Augenheilkd 179:169-172. https://doi.org/10. 1055/s-2008-1057284 (\#162)

44. Strasburger H, Remky A, Murray IJ et al (1996) Objective measurement of contrast sensitivity and visual acuity with the steady-state visual evoked potential. Ger J Ophthalmol 5:42-52 (\#222)

45. Tyler CW, Apkarian P, Nakayama K (1978) Multiple spatial-frequency tuning of electrical responses from human visual cortex. Exp Brain Res 33:535-550. https:// doi.org/10.1007/BF00235573

46. Strasburger H (1987) The analysis of steady state evoked potentials revisited. Clin Vis Sci 1:245-256

47. Bach M, Joost W (1989) VEP vs spatial frequency at high contrast: Subjects have either a bimodal or single-peaked response function. In: Kulikowski J, Dickinson C, Murray I (eds) Seeing contour and colour. Pergamon Press, Oxford, pp 478-484

48. Tyler CW, Apkarian PA (1985) Effects of contrast, orientation and binocularity in the pattern evoked potential. Vis Res 25:755-766 (\#290)

49. Strasburger H, Scheidler W, Rentschler I (1988) Amplitude and phase characteristics of the steady-state visual evoked potential. Appl Opt 27:1069-1088

50. Douthwaite WA, Jenkins TC (1982) Visually evoked responses to checkerboard patterns: check and field size interactions. Am J Optom Physiol Opt 59:894-901 (\#212)

51. Cobb WA, Ettlinger G, Morton HB (1968) Cerebral potentials evoked in man by pattern reversal and their suppression in visual rivalry. J Physiol 195:33P-34P

52. Parry NRA, Murray IJ, Hadjizenonos C (1999) Spatiotemporal tuning of VEPs: effect of mode of stimulation. Vis Res 39:3491-3497. https://doi.org/10.1016/S00426989(99)00098-X

53. Howe JW, Mitchell KW, Robson C (1981) Electrophysiological assessment of visual acuity. Trans Ophthalmol Soc U K 101:105-108 (\#44)

54. Hajek A, Zrenner E (1988) Improved objective visual assessment with visual evoked cortical potentials by rapid pattern stimuli sequences of different spatial frequency. Fortschr Ophthalmol 85:550-554 (\#10)

55. Ridder WH (2004) Methods of visual acuity determination with the spatial frequency sweep visual evoked potential. Doc Ophthalmol 109:239-247 (\#27)

56. Blakemore C, Campbell FW (1969) On the existence of neurones in the human visual system selectively sensitive 
to the orientation and size of retinal images. J Physiol 203:237-260. https://doi.org/10.1113/jphysiol.1969. sp008862

57. Stromeyer CF, Julesz B (1972) Spatial-frequency masking in vision: critical bands and spread of masking*. J Opt Soc Am, JOSA 62:1221-1232. https://doi.org/10.1364/JOSA. 62.001221

58. Campbell FW, Robson JG (1968) Application of Fourier analysis to the visibility of gratings. J Physiol 197:551-566

59. Regan D (1983) Spatial frequency mechanisms in human vision investigated by evoked potential recording. Vis Res 23:1401-1407. 6989(83)90151-7

https://doi.org/10.1016/0042-

60. Heinrich SP, Bach M (2003) Adaptation characteristics of steady-state motion visual evoked potentials. Clin Neurophysiol 114:1359-1366

61. Ho WA, Berkley MA (1988) Evoked potential estimates of the time course of adaptation and recovery to counterphase gratings. Vis Res 28:1287-1296. https://doi.org/10.1016/ 0042-6989(88)90059-4

62. Peachey NS, Demarco PJ, Ubilluz R, Yee W (1994) Shortterm changes in the response characteristics of the human visual evoked potential. Vis Res 34:2823-2831. https:// doi.org/10.1016/0042-6989(94)90051-5

63. Regan D (1977) Steady-state evoked potentials. J Opt Soc Am 67:1475-1489

64. DeValois RL, DeValois KK (1990) Chpt 6 multiple spatial frequency channels. In: Spatial vision. Oxford University Press

65. Orel-Bixler D, Haegerstrom-Portnoy G, Hall A (1989) Visual assessment of the multiply handicapped patient. Optom Vis Sci 66:530-536 (\#86)

66. Odom JV, Bach M, Brigell M et al (2016) ISCEV standard for clinical visual evoked potentials: (2016 update). Doc Ophthalmol 133:1-9. https://doi.org/10.1007/s10633-0169553-y

67. Manahilov V, Riemslag FCC, Spekreijse H (1992) The laplacian analysis of the pattern onset response in man. Electroencephalogr Clin Neurophysiol 82:220-224. https://doi.org/10.1016/0013-4694(92)90171-D

68. Mackay A, Bradnam M, Hamilton R (2003) Rapid detection of threshold VEPs. Clin Neurophysiol 114:1009-1020

69. Kurtenbach A, Langrová H, Messias A et al (2013) A comparison of the performance of three visual evoked potential-based methods to estimate visual acuity. Doc Ophthalmol 126:45-56. https://doi.org/10.1007/s10633012-9359-5 (\#245)

70. Beers AP, Riemslag FC, Spekreijse H (1992) Visual evoked potential estimation of visual activity with a Laplacian derivation. Doc Ophthalmol 79:383-389 (\#262)

71. Hou C, Norcia AM, Madan A, Good WV (2014) Visuocortical function in infants with a history of neonatal jaundice. Invest Ophthalmol Vis Sci 55:6443-6449. https://doi.org/10.1167/iovs.14-14261 (\#207)

72. Pei F, Baldassi S, Norcia AM (2014) Electrophysiological measures of low-level vision reveal spatial processing deficits and hemispheric asymmetry in autism spectrum disorder. J Vis. https://doi.org/10.1167/14.11.3 (\#247)

73. Salomão SR, Ejzenbaum F, Berezovsky A et al (2008) Age norms for monocular grating acuity measured by sweep-
VEP in the first three years of age. Arq Bras Oftalmol 71:475-479 (\#279)

74. Cavascan NN, Salomão SR, Sacai PY et al (2014) Contributing factors to VEP grating acuity deficit and interocular acuity difference in children with cerebral visual impairment. Doc Ophthalmol 128:91-99. https://doi.org/ 10.1007/s10633-013-9423-9 (\#36)

75. Watson T, Orel-Bixler D, Haegerstrom-Portnoy G (2009) VEP vernier, VEP grating, and behavioral grating acuity in patients with cortical visual impairment. Optom Vis Sci 86:774-780. https://doi.org/10.1097/OPX. 0b013e3181a59d2a (\#109)

76. Kharauzov AK, Pronin SV, Sobolev AF et al (2006) Objective measurement of human visual acuity by visual evoked potentials. Neurosci Behav Physiol 36:1021-1030. https://doi.org/10.1007/s11055-006-0139-0 (\#193)

77. Tinelli F, Pei F, Guzzetta A et al (2008) The assessment of visual acuity in children with periventricular damage: a comparison of behavioural and electrophysiological techniques. Vis Res 48:1233-1241. https://doi.org/10.1016/j. visres.2008.02.009 (\#280)

78. Jenkins TC, Douthwaite WA, Peedle JE (1985) The VER as a predictor of normal visual acuity in the adult human eye. Ophthalmic Physiol Opt 5:441-449 (\#47)

79. Good WV, Hou C, Norcia AM (2012) Spatial contrast sensitivity vision loss in children with cortical visual impairment. Invest Ophthalmol Vis Sci 53:7730-7734. https://doi.org/10.1167/iovs.12-9775 (\#153)

80. Appelbaum LG, Wade AR, Vildavski VY et al (2006) Cue-invariant networks for figure and background processing in human visual cortex. $J$ Neurosci 26:11695-11708. https://doi.org/10.1523/JNEUROSCI. 2741-06.2006

81. Norcia AM, Appelbaum LG, Ales JM et al (2015) The steady-state visual evoked potential in vision research: a review. J Vis 15:4. https://doi.org/10.1167/15.6.4

82. Heinrich SP (2010) Some thoughts on the interpretation of steady-state evoked potentials. Doc Ophthalmol 120:205-214. https://doi.org/10.1007/s10633-010-9212-7

83. O'Connor DL, Weishuhn K, Rovet J et al (2012) Visual development of human milk-fed preterm infants provided with extra energy and nutrients after hospital discharge. JPEN J Parenter Enteral Nutr 36:349-353. https://doi.org/ 10.1177/0148607111414026 (\#312)

84. Zhou P, Zhao M-W, Li X-X et al (2008) A new method of extrapolating the sweep pattern visual evoked potential acuity. Doc Ophthalmol 117:85-91. https://doi.org/10. 1007/s 10633-007-9095-4 (\#148)

85. Meigen T, Bach M (1999) On the statistical significance of electrophysiological steady-state responses. Doc Ophthalmol 98:207-232

86. Peli E, McCormack G, Sokol S (1988) Signal to noise ratio considerations in the analysis of sweep visual-evoked potentials. Appl Opt, AO 27:1094-1098. https://doi.org/ 10.1364/AO.27.001094

87. Seiple W, Holopigian K (1989) An examination of VEP response phase. Electroencephalogr Clin Neurophysiol 73:520-531 (\#302)

88. Orel-Bixler DA (1989) Subjective and visual-evoked potential measures of acuity in normal and amblyopic 
adults and children. University of California, California (\#55)

89. Hotelling H (1931) The generalization of student's ratio. Ann Math Stat 2:360-378. https://doi.org/10.1214/aoms/ 1177732979

90. Victor JD, Mast J (1991) A new statistic for steady-state evoked potentials. Electroencephalogr Clin Neurophysiol 78:378-388. 4694(91)90099-P https://doi.org/10.1016/0013-

91. Dobie RA, Wilson MJ (1993) Objective response detection in the frequency domain. Electroencephalogr Clin Neurophysiol/Evok Potent Sect 88:516-524. https://doi. org/10.1016/0168-5597(93)90040-V

92. Mackay AM, Bradnam MS, Hamilton R et al (2008) Realtime rapid acuity assessment using VEPs: development and validation of the step VEP technique. Invest Ophthalmol Vis Sci 49:438-441. https://doi.org/10.1167/iovs. 06-0944 (\#281)

93. Bradnam MS, Mackay AM, Hamilton R (2002) The circular T-squared statistic and SNR measurement are complementary for fast objective detection of steady-state visual evoked potentials (VEPs). In: IEE Proceedings, London, pp 3-7

94. Mackay AM, Hamilton R, Bradnam MS (2003) Faster and more sensitive VEP recording in children. Doc Ophthalmol 107:251-259. https://doi.org/10.1023/B:DOOP. 0000005334.70304.c7

95. Hamilton R, Bradnam MS, Dutton GN et al (2013) Sensitivity and specificity of the step VEP in suspected functional visual acuity loss. Doc Ophthalmol 126:99-104. https://doi.org/10.1007/s10633-012-9362-x (\#243)

96. Towle VL, Harter MR (1977) Objective determination of human visual acuity: pattern evoked potentials. Invest Ophthalmol Vis Sci 16:1073-1076 (\#40)

97. Raniel Y, Pratt H, Neumann E, Schacham SE (1989) Miniature fiber-optic pattern-reversal stimulator for determination of the visual evoked potential threshold; comparison with Snellen acuity. Graefes Arch Clin Exp Ophthalmol 227:212-215 (\#87)

98. Towle VL, Harter MR (1979) Objective determination of human visual acuity from the visual evoked potential. Percept Psychophys 25:497-500 (\#210)

99. Nakamura A, Akio T, Matsuda E, Wakami Y (2001) Pattern visual evoked potentials in malingering. J Neuroophthalmol 21:42-45 (\#231)

100. McBain VA, Robson AG, Hogg CR, Holder GE (2007) Assessment of patients with suspected non-organic visual loss using pattern appearance visual evoked potentials. Graefes Arch Clin Exp Ophthalmol 245:502-510. https:// doi.org/10.1007/s00417-006-0431-2 (\#237)

101. Mitsuyu M, Zimmer EM (1984) Bangerter occlusives versus spherical convex lenses in the evaluation of visual acuity by visually evoked cortical potentials. Dev Ophthalmol 9:115-122 (\#252)

102. Strasser T, Nasser F, Langrová H et al (2019) Objective assessment of visual acuity: a refined model for analyzing the sweep VEP. Doc Ophthalmol 138:97-116. https://doi. org/10.1007/s10633-019-09672-z (\#325)

103. Keyser MD, Vissenberg I, Neetens A (1990) Are visually evoked potentials (VEP) useful for determination of visual acuity?: a clinical trial. Neuro-Ophthalmology
10:153-163. https://doi.org/10.3109/01658109008997277 (\#216)

104. Fisher AC, McCulloch DL, Borchert MS et al (2015) Comparison of human expert and computer-automated systems using magnitude-squared coherence (MSC) and bootstrap distribution statistics for the interpretation of pattern electroretinograms (PERGs) in infants with optic nerve hypoplasia (ONH). Doc Ophthalmol 131:25-34. https://doi.org/10.1007/s10633-015-9493-y

105. Grall MY, Rigaudière F, Delthil S, Keller J (1976) A methods of objective measurement of visual acuity based on the study of V.E.P. Bull Soc Ophtalmol Fr 76:1109 (\#1)

106. Iyer KK, Bradley AP, Wilson SJ (2013) Conducting shorter VEP tests to estimate visual acuity via assessment of SNR. Doc Ophthalmol 126:21-28. https://doi.org/10. 1007/s10633-012-9355-9 (\#244)

107. Lv J, Simpson DM, Bell SL (2007) Objective detection of evoked potentials using a bootstrap technique. Med Eng Phys 29:191-198. https://doi.org/10.1016/j.medengphy. 2006.03.001

108. Harter MR, White CT (1970) Evoked cortical responses to checkerboard patterns: effect of check-size as a function of visual acuity. Electroencephalogr Clin Neurophysiol 28:48-54. https://doi.org/10.1016/0013-4694(70)90007-6

109. Heine S, Rüther K, Isensee J, Zrenner E (1999) Clinical significance of objective vision assessment using visually evoked cortical potentials induced by rapid pattern sequences of different spatial frequency. Klin Monbl Augenheilkd 215:175-181. https://doi.org/10.1055/s2008-1034695 (\#65)

110. van der Marel H, van Beem H, Spekreijse H (1982) Nitrous oxide influences pattern EP estimate of visual acuity. Graefes Arch Clin Exp Ophthalmol 218:250-252 (\#45)

111. Gräf M, Dettmar T, Kaufmann H (1996) Objective determination of visual acuity: improvement of an infrared nystagmography method and comparison with pattern visual evoked potentials. Ophthalmologe 93:396-403 (\#61)

112. Heinrich SP, Bock CM, Bach M (2016) Imitating the effect of amblyopia on VEP-based acuity estimates. Doc Ophthalmol 133:183-187. https://doi.org/10.1007/s10633016-9565-7 (\#287)

113. Marechal L, Faidherbe J (1990) Trial on the objective evaluation of visual acuity and its maturation by the method of evoked visual potentials. Arch Int Physiol Biochim 98:TD1-96 (\#217)

114. Campbell FW, Green DG (1965) Optical and retinal factors affecting visual resolution. J Physiol 181:576-593. https://doi.org/10.1113/jphysiol.1965.sp007784

115. Campbell FW, Kulikowski JJ (1972) The visual evoked potential as a function of contrast of a grating pattern. J Physiol 222:345-356. https://doi.org/10.1113/jphysiol. 1972.sp009801

116. Wiener DE, Wellish K, Nelson JI, Kupersmith MJ (1985) Comparisons among Snellen, psychophysical, and evoked potential visual acuity determinations. Am J Optom Physiol Opt 62:669-679 (\#46)

117. Tyler CW, Nakayama K, Apkarian PA, Levi DM (1981) VEP assessment of visual function. Vis Res 21:607-609. https://doi.org/10.1016/0042-6989(81)90109-7 
118. Ohn YH, Katsumi O, Matsui Y et al (1994) Snellen visual acuity versus pattern reversal visual-evoked response acuity in clinical applications. Ophthalmic Res 26:240-252. https://doi.org/10.1159/000267482 (\#95)

119. Bach M, Meigen T (1999) Do's and don'ts in Fourier analysis of steady-state potentials. Doc Ophthalmol 99:69-82

120. Katsumi O, Arai M, Wajima R et al (1996) Spatial frequency sweep pattern reversal VER acuity vs Snellen visual acuity: effect of optical defocus. Vis Res 36:903-909 (\#62)

121. Kromer R, Serbecic N, Krastel H, Beutelspacher SC (2014) Comparison of VEP with contrast sensitivity and other measurements of central visual function. Acta Ophthalmol 92:e141-146. https://doi.org/10.1111/aos. 12176 (\#117)

122. Sokol S, Moskowitz A, McCormack G (1992) Infant VEP and preferential looking acuity measured with phase alternating gratings. Invest Ophthalmol Vis Sci 33:3156-3161 (\#131)

123. Norcia AM, Tyler CW (1985) Infant VEP acuity measurements: analysis of individual differences and measurement error. Electroencephalogr Clin Neurophysiol 61:359-369 (\#48)

124. Orel-Bixler DA, Norcia AM (1987) Differential growth of acuity for steady-state pattern reversal and transient pattern onset-offset VEPs. Clin Vis Sci 2:1-9 (\#176)

125. Suttle CM, Banks MS, Candy TR (2000) Does a front-end nonlinearity confound VEP acuity measures in human infants? Vis Res 40:3665-3675 (\#269)

126. Good WV, Hou C (2006) Sweep visual evoked potential grating acuity thresholds paradoxically improve in lowluminance conditions in children with cortical visual impairment. Invest Ophthalmol Vis Sci 47:3220-3224. https://doi.org/10.1167/iovs.05-1252 (\#235)

127. Hamer RD, Norcia AM, Tyler CW, Hsu-Winges C (1989) The development of monocular and binocular VEP acuity. Vis Res 29:397-408 (\#51)

128. Atkinson J, Braddick O, French J (1979) Contrast sensitivity of the human neonate measured by the visual evoked potential. Invest Ophthalmol Vis Sci 18:210-213 (\#293)

129. Riddell PM, Ladenheim B, Mast J et al (1997) Comparison of measures of visual acuity in infants: Teller acuity cards and sweep visual evoked potentials. Optom Vis Sci 74:702-707 (\#21)

130. Marg E, Freeman D, Peltzman P, Goldstein P (1976) Visual acuity development in human infants: evoked potential measurements. Invest Ophthalmol Vis Sci 15:150-153 (\#328)

131. Prager TC, Zou YL, Jensen CL et al (1999) Evaluation of methods for assessing visual function of infants. J AAPOS 3:275-282 (\#22)

132. Buckley SW (1997) A novel swept-parameter transient visual evoked potential technique. Yeshiva University, New York (\#63)

133. Hoffman DR, Theuer RC, Castañeda YS et al (2004) Maturation of visual acuity is accelerated in breast-fed term infants fed baby food containing DHA-enriched egg yolk. J Nutr 134:2307-2313. https://doi.org/10.1093/jn/ 134.9.2307 (\#66)
134. Jensen CL, Voigt RG, Llorente AM et al (2010) Effects of early maternal docosahexaenoic acid intake on neuropsychological status and visual acuity at five years of age of breast-fed term infants. J Pediatr 157:900-905. https://doi. org/10.1016/j.jpeds.2010.06.006 (\#71)

135. Hou C, Norcia AM, Madan A et al (2011) Visual cortical function in very low birth weight infants without retinal or cerebral pathology. Invest Ophthalmol Vis Sci 52:9091-9098. https://doi.org/10.1167/iovs.11-7458 (\#73)

136. Hoffman DR, Birch EE, Castañeda YS et al (2003) Visual function in breast-fed term infants weaned to formula with or without long-chain polyunsaturates at 4 to 6 months: a randomized clinical trial. J Pediatr 142:669-677. https:// doi.org/10.1067/mpd.2003.213 (\#99)

137. Birch EE, Castañeda YS, Wheaton DH et al (2005) Visual maturation of term infants fed long-chain polyunsaturated fatty acid-supplemented or control formula for $12 \mathrm{mo}$. Am J Clin Nutr 81:871-879. https://doi.org/10.1093/ajen/81.4. 871 (\#102)

138. Birch EE, Carlson SE, Hoffman DR et al (2010) The DIAMOND (DHA Intake And Measurement Of Neural Development) Study: a double-masked, randomized controlled clinical trial of the maturation of infant visual acuity as a function of the dietary level of docosahexaenoic acid. Am J Clin Nutr 91:848-859. https://doi.org/10.3945/ ajcn.2009.28557 (\#110)

139. Sokol S (1978) Measurement of infant visual acuity from pattern reversal evoked potentials. Vis Res 18:33-39 (\#118)

140. Jørgensen MH, Hølmer G, Lund P et al (1998) Effect of formula supplemented with docosahexaenoic acid and gamma-linolenic acid on fatty acid status and visual acuity in term infants. J Pediatr Gastroenterol Nutr 26:412-421 (\#137)

141. Sokol S, Moskowitz A, McCormack G, Augliere R (1988) Infant grating acuity is temporally tuned. Vis Res 28:1357-1366 (\#215)

142. Suter S, Suter PS, Crow CD (1991) Infant and adult grating acuity estimated by VEPs and heart-rate change. Infant Behav Dev 14:365-382. https://doi.org/10.1016/01636383(91)90028-Q (\#218)

143. Birch E, Birch D, Hoffman D et al (1993) Breast-feeding and optimal visual development. J Pediatr Ophthalmol Strabismus 30:33-38 (\#220)

144. Birch EE, Hoffman DR, Uauy R et al (1998) Visual acuity and the essentiality of docosahexaenoic acid and arachidonic acid in the diet of term infants. Pediatr Res 44:201-209. https://doi.org/10.1203/00006450199808000-00011 (\#226)

145. Gibson RA, Neumann MA, Makrides M (1997) Effect of increasing breast milk docosahexaenoic acid on plasma and erythrocyte phospholipid fatty acids and neural indices of exclusively breast fed infants. Eur J Clin Nutr 51:578-584 (\#296)

146. Lauritzen L, Jørgensen MH, Mikkelsen TB et al (2004) Maternal fish oil supplementation in lactation: effect on visual acuity and n-3 fatty acid content of infant erythrocytes. Lipids 39:195-206 (\#297)

147. Smithers LG, Gibson RA, Makrides M (2011) Maternal supplementation with docosahexaenoic acid during 
pregnancy does not affect early visual development in the infant: a randomized controlled trial. Am J Clin Nutr 93:1293-1299. https://doi.org/10.3945/ajen.110.009647 (\#303)

148. Mirabella G, Kjaer PK, Norcia AM et al (2006) Visual development in very low birth weight infants. Pediatr Res 60:435-439. https://doi.org/10.1203/01.pdr.0000238249. 44088.2c (\#311)

149. Auestad N, Montalto MB, Hall RT et al (1997) Visual acuity, erythrocyte fatty acid composition, and growth in term infants fed formulas with long chain polyunsaturated fatty acids for one year. Ross Pediatric Lipid Study. Pediatr Res 41:1-10. https://doi.org/10.1203/00006450199701000-00001 (\#317)

150. Polevoy C, Muckle G, Séguin JR et al (2017) Similarities and differences between behavioral and electrophysiological visual acuity thresholds in healthy infants during the second half of the first year of life. Doc Ophthalmol 134:99-110. https://doi.org/10.1007/s10633-017-9576-z (\#323)

151. Sokol S, Moskowitz A (1985) Comparison of pattern VEPs and preferential-looking behavior in 3-month-old infants. Invest Ophthalmol Vis Sci 26:359-365 (\#172)

152. Makrides M, Simmer K, Goggin M, Gibson RA (1993) Erythrocyte docosahexaenoic acid correlates with the visual response of healthy, term infants. Pediatr Res 33:425-427. https://doi.org/10.1203/00006450199304000-00021 (\#320)

153. Makrides M, Neumann M, Simmer K et al (1995) Are long-chain polyunsaturated fatty acids essential nutrients in infancy? Lancet 345:1463-1468. https://doi.org/10. 1016/s0140-6736(95)91035-2 (\#183)

154. Makrides M, Neumann MA, Simmer K, Gibson RA (2000) A critical appraisal of the role of dietary long-chain polyunsaturated fatty acids on neural indices of term infants: a randomized, controlled trial. Pediatrics 105:32-38. https://doi.org/10.1542/peds.105.1.32 (\#187)

155. Till C, Westall CA, Koren G et al (2005) Vision abnormalities in young children exposed prenatally to organic solvents. Neurotoxicology 26:599-613. https://doi.org/10. 1016/j.neuro.2005.05.011 (\#304)

156. Mezer E, Westall CA, Mirabella G et al (2016) Measuring recovery of visual function in children with papilledema using sweep visual evoked potentials. J AAPOS 20:252-257. https://doi.org/10.1016/j.jaapos.2016.03.010 (\#300)

157. Thompson DA, Møller H, Russell-Eggitt I, Kriss A (1996) Visual acuity in unilateral cataract. $\mathrm{Br} \mathrm{J}$ Ophthalmol 80:794-798. https://doi.org/10.1136/bjo.80.9.794 (\#329)

158. John FM, Bromham NR, Woodhouse JM, Candy TR (2004) Spatial vision deficits in infants and children with Down syndrome. Invest Ophthalmol Vis Sci 45:1566-1572. https://doi.org/10.1167/iovs.03-0951 (\#288)

159. Norcia AM, Clarke M, Tyler CW (1985) Digital filtering and robust regression techniques for estimating sensory thresholds from the evoked potential. IEEE Eng Med Biol Mag 4:26-32. https://doi.org/10.1109/MEMB.1985. 5006224

160. Norcia AM, Tyler CW, Piecuch R et al (1987) Visual acuity development in normal and abnormal preterm human infants. J Pediatr Ophthalmol Strabismus 24:70-74 (\#50)

161. Birch E, Birch D, Petrig B, Uauy R (1990) Retinal and cortical function of very low birthweight infants at 36 and 57 weeks postconception. Clin Vis Sci 5:363-373 (\#259)

162. Birch EE, Birch DG, Hoffman DR, Uauy R (1992) Dietary essential fatty acid supply and visual acuity development. Invest Ophthalmol Vis Sci 33:3242-3253 (\#179)

163. Smithers LG, Gibson RA, McPhee A, Makrides M (2008) Higher dose of docosahexaenoic acid in the neonatal period improves visual acuity of preterm infants: results of a randomized controlled trial. Am J Clin Nutr 88:1049-1056. https://doi.org/10.1093/ajcn/88.4.1049 (\#147)

164. O'Connor DL, Hall R, Adamkin D et al (2001) Growth and development in preterm infants fed long-chain polyunsaturated fatty acids: a prospective, randomized controlled trial. Pediatrics 108:359-371. https://doi.org/10.1542/ peds.108.2.359 (\#141)

165. Madan A, Norcia AM, Hou C et al (2012) Effect of Grade I and II intraventricular hemorrhage on visuocortical function in very low birth weight infants. See Perceiving 25:143-154. https://doi.org/10.1163/187847612X626381 (\#154)

166. Kos-Pietro S, Towle VL, Cakmur R, Spire JP (1997) Maturation of human visual evoked potentials: 27 weeks conceptional age to 2 years. Neuropediatrics 28:318-323. https://doi.org/10.1055/s-2007-973723 (\#225)

167. Li L, Su Y, Chen C et al (2011) Sweep pattern visual evoked potential acuity in children during their periods of visual development. Ophthalmologica 226:220-227. https://doi.org/10.1159/000329866 (\#242)

168. Stadler G, Muller J (1982) Determination of visual acuity with pattern evoked cortical responses. Doc Ophthalmol Proc Ser 31:437-442 (\#165)

169. Petersen J (1984) Objective determination of visual acuity by visual evoked potentials: optimized procedure and clinical value. Dev Ophthalmol 9:108-114 (\#7)

170. Bach M, Waltenspiel S, Schildwächter A (1989) Detection of defocused gratings: spurious resolution, a pitfall in the determination of visual acuity based on preferential looking or VEP. In: Kulikowski J, Dickinson C, Murray I (eds) Seeing contour and colour. Pergamon, Oxford, pp 562-565

171. Strasburger H, Bach M, Heinrich SP (2018) Blur Unblurred-A Mini Tutorial. Iperception 9:2041669518765850. https://doi.org/10.1177/2041669518765850

172. Arai M, Katsumi O, Paranhos FR et al (1997) Comparison of Snellen acuity and objective assessment using the spatial frequency sweep PVER. Graefes Arch Clin Exp Ophthalmol 235:442-447 (\#97)

173. Gottlob I, Fendick MG, Guo S et al (1990) Visual acuity measurements by swept spatial frequency visual-evokedcortical potentials (VECPs): clinical application in children with various visual disorders. J Pediatr Ophthalmol Strabismus 27:40-47 (\#55)

174. Sokol S, Hansen VC, Moskowitz A et al (1983) Evoked potential and preferential looking estimates of visual acuity in pediatric patients. Ophthalmology 90:552-562 (\#166) 
175. Katsumi O, Denno S, Arai M et al (1997) Comparison of preferential looking acuity and pattern reversal visual evoked response acuity in pediatric patients. Graefes Arch Clin Exp Ophthalmol 235:684-690 (\#20)

176. Bane MC, Birch EE (1992) VEP acuity, FPL acuity, and visual behavior of visually impaired children. J Pediatr Ophthalmol Strabismus 29:202-209 (\#59)

177. Katsumi O, Mehta MC, Larson-Park EW et al (1994) Pattern reversal visual evoked response and Snellen visual acuity. Graefes Arch Clin Exp Ophthalmol 232:272-278 (\#263)

178. Teping C (1980) Klinische Anwendung des visuell evozierten kortikalen Potentials (VECP) zur Visusbestimmung. In: Jaeger W (ed) Plastische Chirurgie der Lider und Chirurgie der Tränenwege. J.F. Bergmann-Verlag, pp 399-403. (\#81)

179. McCulloch DL, Skarf B (1994) Pattern reversal visual evoked potentials following early treatment of unilateral, congenital cataract. Arch Ophthalmol 112:510-518 (\#264)

180. Creel D, Spekreijse H, Reits D (1981) Visual evoked potential (VEP) methods of detecting misrouted optic projections. In: Spekreijse H, Apkarian PA (eds) Visual pathways: electrophysiology and pathology. Springer, Dordrecht, pp 157-165

181. Saunders KJ, Brown G, McCulloch DL (1997) Patternonset visual evoked potentials: more useful than reversal for patients with nystagmus. Doc Ophthalmol 94:265-274. https://doi.org/10.1007/BF02582984

182. Hoffmann MB, Seufert PS, Bach M (2004) Simulated nystagmus suppresses pattern-reversal but not patternonset visual evoked potentials. Clin Neurophysiol 115:2659-2665. https://doi.org/10.1016/j.clinph.2004.06. 003

183. Westall CA, Ainsworth JR, Buncic JR (2000) Which ocular and neurologic conditions cause disparate results in visual acuity scores recorded with visually evoked potential and teller acuity cards? J AAPOS 4:295-301. https:// doi.org/10.1067/mpa.2000.107898 (\#186)

184. Bane MC, Birch EE (1992) Forced-choice preferential looking and visual evoked potential acuities of visually impaired children. J Vis Impair Blind 86:21-24 (\#295)

185. Meiusi RS, Lavoie JD, Summers CG (1993) The effect of grating orientation on resolution acuity in patients with nystagmus. J Pediatr Ophthalmol Strabismus 30:259-261. https://doi.org/10.3928/0191-3913-19930701-09

186. Gottlob I, Wizov S, Odom J, Reinecke R (1993) Predicting optotype visual acuity by swept spatial visual-evoked potentials. Clin Vis Sci 8:417-423 (\#132)

187. El-Gohary AM, Sabry HN (2014) Determination of visual acuity in children using sweep visual evoked potential compared to conventional measuring techniques. Egy J Neurol Psychiat Neurosurg 51:405-411 (\#37)

188. Skalka HW (1980) Comparison of Snellen acuity, VER acuity, and Arden grating scores in macular and optic nerve diseases. Br J Ophthalmol 64:24-29. https://doi.org/ 10.1136/bjo.64.1.24 (\#43)

189. Niermann F, Lorenz R, Heider W (1989) Pattern electroretinography and pattern visual evoked potentials in maculopathy: a comparison with sensory tests. Fortschr Ophthalmol 86:54-58 (\#53)
190. Odom JV, Hoyt CS, Marg E (1981) Effect of natural deprivation and unilateral eye patching on visual acuity of infants and children: evoked potential measurements. Arch Ophthalmol 99:1412-1416 (\#211)

191. Odom JV, Hoyt CS, Marg E (1982) Eye patching and visual evoked potential acuity in children 4 months to eight years old. Am J Optom Physiol Opt 59:706-717 (\#313)

192. Ridder WH, Rouse MW (2007) Predicting potential acuities in amblyopes: predicting post-therapy acuity in amblyopes. Doc Ophthalmol 114:135-145. https://doi.org/ 10.1007/s10633-007-9048-y (\#33)

193. Wenner Y, Heinrich SP, Beisse C et al (2014) Visual evoked potential-based acuity assessment: overestimation in amblyopia. Doc Ophthalmol 128:191-200. https://doi. org/10.1007/s10633-014-9432-3 (\#35)

194. McKee SP, Levi DM, Movshon JA (2003) The pattern of visual deficits in amblyopia. J Vis 3:5. https://doi.org/10. $1167 / 3.5 .5$

195. Hou C, Good WV, Norcia AM (2007) Validation study of VEP vernier acuity in normal-vision and amblyopic adults. Invest Ophthalmol Vis Sci 48:4070-4078. https://doi.org/ 10.1167/iovs.06-1368

196. Costa MF, Ventura DF (2012) Visual impairment in children with spastic cerebral palsy measured by psychophysical and electrophysiological grating acuity tests. Dev Neurorehabil 15:414-424. https://doi.org/10.3109/ 17518423.2012.703704 (\#202)

197. Ghasia F, Brunstom J, Tychsen L (2009) Visual acuity and visually evoked responses in children with cerebral palsy: gross Motor Function Classification Scale. Br J Ophthalmol 93:1068-1072. https://doi.org/10.1136/bjo.2008. 156372 (\#68)

198. Mackie RT, McCulloch DL, Saunders KJ et al (1998) Relation between neurological status, refractive error, and visual acuity in children: a clinical study. Dev Med Child Neurol 40:31-37 (\#227)

199. Saunders KJ, McCulloch DL, Kerr AM (1995) Visual function in rett syndrome. Dev Med Child Neurol 37:496-504. https://doi.org/10.1111/j.1469-8749.1995. tb12037.x

200. da Costa MF, Salomão SR, Berezovsky A et al (2004) Relationship between vision and motor impairment in children with spastic cerebral palsy: new evidence from electrophysiology. Behav Brain Res 149:145-150 (\#143)

201. de Freitas Dotto P, Cavascan NN, Berezovsky A et al (2014) Sweep visually evoked potentials and visual findings in children with West syndrome. Eur $\mathrm{J}$ Paediatr Neurol 18:201-210. https://doi.org/10.1016/j.ejpn.2013. 11.008 (\#155)

202. Wygnanski-Jaffe T, Panton CM, Buncic JR, Westall CA (2009) Paradoxical robust visual evoked potentials in young patients with cortical blindness. Doc Ophthalmol 119:101-107. https://doi.org/10.1007/s10633-009-9176-7 (\#308)

203. Sakki HEA, Dale NJ, Sargent J et al (2018) Is there consensus in defining childhood cerebral visual impairment? A systematic review of terminology and definitions. Br J Ophthalmol 102:424-432. https://doi.org/10.1136/ bjophthalmol-2017-310694 
204. Good WV (2001) Development of a quantitative method to measure vision in children with chronic cortical visual impairment. Trans Am Ophthalmol Soc 99:253-269 (\#190)

205. Skoczenski AM, Good WV (2004) Vernier acuity is selectively affected in infants and children with cortical visual impairment. Dev Med Child Neurol 46:526-532 (\#233)

206. Lim M, Soul JS, Hansen RM et al (2005) Development of visual acuity in children with cerebral visual impairment. Arch Ophthalmol 123:1215-1220. https://doi.org/10. 1001/archopht.123.9.1215 (\#103)

207. Watson T, Orel-Bixler D, Haegerstrom-Portnoy G (2010) Early visual-evoked potential acuity and future behavioral acuity in cortical visual impairment. Optom Vis Sci 87:80-86. https://doi.org/10.1097/OPX. 0b013e3181c75184 (\#241)

208. Enzenauer R, Morris W, O’Donnell T, Montrey J (2014) Terminology of functional disorders. In: Enzenauer R, Morris W, O'Donnell T, Montrey J (eds) Functional ophthalmic disorders: ocular malingering and visual hysteria. Springer, Cham, pp 1-10

209. Wildberger H, Huber C (1980) Visual evoked potentials in the evaluation of suspected simulation and aggravation. Klin Monbl Augenheilkd 176:704-707. https://doi.org/10. 1055/s-2008-1057538 (\#42)

210. Nakamura A, Tabuchi A, Matsuda E, Yamaguchi W (2000) Dynamic topography of pattern visual evoked potentials (PVEP) in psychogenic visual loss patients. Doc Ophthalmol 101:95-113 (\#139)

211. Day SH, Orel-Bixler DA, Norcia AM (1988) Abnormal acuity development in infantile esotropia. Invest Ophthalmol Vis Sci 29:327-329 (\#127)

212. Bradfield YS, France TD, Verhoeve J, Gangnon RE (2007) Sweep visual evoked potential testing as a predictor of recognition acuity in albinism. Arch Ophthalmol 125:628-633. https://doi.org/10.1001/archopht.125.5.628 (\#106)

213. Durbin S, Mirabella G, Buncic JR, Westall CA (2009) Reduced grating acuity associated with retinal toxicity in children with infantile spasms on vigabatrin therapy. Invest Ophthalmol Vis Sci 50:4011-4016. https://doi.org/ 10.1167/iovs.08-3237 (\#239)

214. McCulloch DL, Marmor MF, Brigell MG et al (2015) ISCEV Standard for full-field clinical electroretinography (2015 update). Doc Ophthalmol 130:1-12. https://doi.org/ 10.1007/s10633-014-9473-7

215. Hood DC, Bach M, Brigell M et al (2012) ISCEV standard for clinical multifocal electroretinography (mfERG) (2011 edition). Doc Ophthalmol 124:1-13. https://doi.org/10. 1007/s10633-011-9296-8

216. Bach M, Brigell MG, Hawlina M et al (2013) ISCEV standard for clinical pattern electroretinography (PERG): 2012 update. Doc Ophthalmol 124:1-13. https://doi.org/ 10.1007/s10633-012-9353-y

217. Constable PA, Bach M, Frishman LJ et al (2017) ISCEV Standard for clinical electro-oculography (2017 update). Doc Ophthalmol 134:1-9. https://doi.org/10.1007/s10633017-9573-2

218. Brigell M, Bach M, Barber C, et al (2003) Guidelines for calibration of stimulus and recording parameters used in clinical electrophysiology of vision. Doc Ophthalmol 185-193

219. Robson AG, Nilsson J, Li S et al (2018) ISCEV guide to visual electrodiagnostic procedures. Doc Ophthalmol 136:1-26. https://doi.org/10.1007/s10633-017-9621-y

220. Frishman L, Sustar M, Kremers J et al (2018) ISCEV extended protocol for the photopic negative response (PhNR) of the full-field electroretinogram. Doc Ophthalmol 136:207-211. https://doi.org/10.1007/s10633-0189638-x

221. Johnson MA, Jeffrey BG, Messias AMV, Robson AG (2019) ISCEV extended protocol for the stimulus-response series for the dark-adapted full-field ERG b-wave. Doc Ophthalmol 138:217-227. https://doi.org/10.1007/ s10633-019-09687-6

222. McCulloch DL, Kondo M, Hamilton R et al (2019) ISCEV extended protocol for the stimulus-response series for light-adapted full-field ERG. Doc Ophthalmol 138:205-215. https://doi.org/10.1007/s10633-019-096858

223. Sustar M, Holder GE, Kremers J et al (2018) ISCEV extended protocol for the photopic On-Off ERG. Doc Ophthalmol 136:199-206. https://doi.org/10.1007/ s10633-018-9645-y

224. Thompson DA, Fujinami K, Perlman I et al (2018) ISCEV extended protocol for the dark-adapted red flash ERG. Doc Ophthalmol 136:191-197. https://doi.org/10.1007/ s10633-018-9644-z

225. Hamilton R, Bach M, Heinrich SP et al (2020) ISCEV extended protocol for VEP methods of estimation of visual acuity. Documenta Ophthalmologica [in press]

226. Heinrich SP, Marhöfer D, Bach M (2010) "Cognitive" visual acuity estimation based on the event-related potential P300 component. Clin Neurophysiol 121:1464-1472. https://doi.org/10.1016/j.clinph.2010.03. 030

227. Bodis-Wollner I, Atkin A, Raab E, Wolkstein M (1977) Visual association cortex and vision in man: patternevoked occipital potentials in a blind boy. Science 198:629-631. https://doi.org/10.1126/science.918658

228. EP28A3C: Define and Verify Reference Intervals in Lab. In: Clinical \& Laboratory Standards Institute. https://clsi. org/standards/products/method-evaluation/documents/ ep28/. Accessed 9 Oct 2019

229. Banks MS, Bennett PJ (1988) Optical and photoreceptor immaturities limit the spatial and chromatic vision of human neonates. J Opt Soc Am A, JOSAA 5:2059-2079. https://doi.org/10.1364/JOSAA.5.002059

230. Dobson V, Teller DY (1978) Visual acuity in human infants: a review and comparison of behavioral and electrophysiological studies. Vis Res 18:1469-1483. https:// doi.org/10.1016/0042-6989(78)90001-9

231. Pompe MT, Liasis A, Hertle R (2019) Visual electrodiagnostics and eye movement recording: World Society of Pediatric Ophthalmology and Strabismus (WSPOS) consensus statement. Indian J Ophthalmol 67:23-30. https:// doi.org/10.4103/ijo.IJO_1103_18

232. Moskowitz A, Sokol S (1983) Developmental changes in the human visual system as reflected by the latency of the pattern reversal VEP. Electroencephalogr Clin 
Neurophysiol 56:1-15. https://doi.org/10.1016/00134694(83)90002-0

233. McCulloch DL, Skarf B (1991) Development of the human visual system: monocular and binocular pattern VEP latency. Invest Ophthalmol Vis Sci 32:2372-2381

234. Meigen T, Kley F (2007) Die adaptive Bestimmung von Kontrastschwellen mit dem visuell evozierten Potenzial (VEP). Zeitschrift für Medizinische Physik 17:24-33. https://doi.org/10.1016/j.zemedi.2006.11.006

235. Allison BZ, McFarland DJ, Schalk G et al (2008) Towards an independent brain-computer interface using steady state visual evoked potentials. Clin Neurophysiol 119:399-408. https://doi.org/10.1016/j.clinph.2007.09.121

236. Zhang Y, Zhou G, Jin J et al (2014) Frequency recognition in SSVEP-based BCI using multiset canonical correlation analysis. Int J Neural Syst 24:1450013. https://doi.org/10. 1142/S0129065714500130
237. Lightbody G, Brennan CP, McCullagh PJ et al (2018) Eye gaze collaboration with brain-computer interfaces: using both modalities for more robust interaction. In: Braincomputer interfaces handbook. https://www.taylorfrancis. com/. Accessed 9 Oct 2019

238. Bach M, Heinrich SP (2019) Acuity VEP: improved with machine learning. Doc Ophthalmol 139:113-122. https:// doi.org/10.1007/s10633-019-09701-x

239. Brouwer A-M, Reuderink B, Vincent J et al (2013) Distinguishing between target and nontarget fixations in a visual search task using fixation-related potentials. J Vis 13:17. https://doi.org/10.1167/13.3.17

Publisher's Note Springer Nature remains neutral with regard to jurisdictional claims in published maps and institutional affiliations. 